\title{
Proof of the classical soft graviton theorem in $D=4$
}

\section{Arnab Priya Saha, Biswajit Sahoo and Ashoke Sen}

Harish-Chandra Research Institute, HBNI,

Chhatnag Road, Jhusi, Allahabad 211019, India

E-mail: arnabpriyasaha@hri.res.in, biswajitsahoo@hri.res.in, sen@hri.res.in

ABSTRACT: Classical subleading soft graviton theorem in four space-time dimensions determines the gravitational wave-form at late and early retarded time, generated during a scattering or explosion, in terms of the four momenta of the ingoing and outgoing objects. This result was 'derived' earlier by taking the classical limit of the quantum soft graviton theorem, and making some assumptions about how to deal with the infrared divergences of the soft factor. In this paper we give a direct proof of this result by analyzing the classical equations of motion of gravity coupled to matter. We also extend the result to the electromagnetic wave-form generated during scattering of charged particles, and present a new conjecture on subsubleading corrections to the gravitational wave-form at early and late retarded time.

Keywords: Scattering Amplitudes, Classical Theories of Gravity, Gauge Symmetry, Space-Time Symmetries

ARXiv EPrint: 1912.06413 


\section{Contents}

1 Introduction and summary 1

2 Some useful results 4

3 Proof of classical soft graviton theorem 5

3.1 General set-up 6

$\begin{array}{ll}3.2 & \text { Leading order contribution }\end{array}$

3.3 First order correction to the gravitational field 9

$\begin{array}{ll}3.4 & \text { Subleading contribution to the matter stress tensor } \\ \end{array}$

$\begin{array}{ll}\text { 3.5 Subleading contribution from the gravitational stress tensor } & 15\end{array}$

3.6 Gravitational wave-form at early and late time 20

4 Generalizations $\quad 21$

4.1 Soft photon theorem with electromagnetic interactions 21

4.2 Gravitational contribution to the soft photon theorem 23

$\begin{array}{lll}4.3 & \text { Electromagnetic wave-form at early and late time } & 25\end{array}$

4.4 Electromagnetic contribution to the soft graviton theorem 25

$5 \quad$ New conjectures at the subsubleading order $\quad 27$

$6 \quad$ Numerical estimate $\quad 30$

A Derivation of some useful mathematical results 32

A.1 Radiative field at large distance 32

A.2 Late and early time behaviour from Fourier transformation 33

$\begin{array}{ll}\text { B Evaluation of some integrals } & 36\end{array}$

$\begin{array}{ll}\text { C Contribution from real gravitons } & 38\end{array}$

$\begin{array}{ll}\text { D Position space analysis of } \widehat{T}^{X \mu \nu} & 41\end{array}$

\section{Introduction and summary}

In a quantum theory of gravity, soft graviton theorem gives an amplitude with a set of finite energy external particles and one or more low energy external gravitons, in terms of the amplitude without the low energy gravitons [1-14]. However when we take the classical limit, there is a different manifestation of the same theorem - it determines the low frequency component of the gravitational wave-form produced during a scattering 
process in terms of the momenta and spin of the incoming and outgoing objects, without any reference to the interactions responsible for the scattering [15]. Although initially this result was derived by taking the classical limit of quantum amplitudes, this has now been proved directly in the classical theory [16] in five or more space-time dimensions.

In four space-time dimensions there are additional subtleties. In quantum theory these are related to infrared divergences of the S-matrix. In the classical limit these manifest themselves in the logarithmic corrections to the asymptotic trajectories of the objects due to the long range force operating between these objects. Due to these logarithmic corrections, the orbital angular momenta of external objects, that enter the expression for the gravitational wave-form, become ill defined. Refs. [17, 18] proposed a specific way of regulating these logarithmic divergences by suggesting that we use the wave-length of the soft graviton as the infrared cut-off. This introduced terms proportional to $\ln \omega$ in the soft graviton theorem where $\omega$ is the angular frequency of the soft graviton. After Fourier transformation, these terms control the gravitational wave-form produced during a scattering at late and early retarded time [19]. ${ }^{1}$

Our goal in this paper will be to prove the classical soft graviton theorem in four space-time dimensions directly in the classical theory. In particular we prove the following result. Let us consider a scattering process in which a set of $m$ objects carrying four momenta $p_{1}^{\prime}, \cdots, p_{m}^{\prime}$ come together, scatter via some (unknown) interactions and disperse as $n$ objects carrying momenta $p_{1}, \cdots, p_{n}$. The special case $m=1$ will describe an explosion in which a single bound system fragments into many objects, including radiation. We shall choose the origin of the space-time coordinate system so that the scattering takes place within a finite neighborhood of the origin. Let us also suppose that we have a gravitational wave detector placed at a faraway point $\vec{x}$, and define

$$
R=|\vec{x}|, \quad \hat{n}=\frac{\vec{x}}{R}, \quad n=(1, \hat{n}) .
$$

We shall consider the limit of large $R$ and analyze only the terms of order $1 / R$ in the gravitational wave-form. We define the retarded time at the detector:

$$
u \equiv t-R+2 G \ln R \sum_{b=1}^{n} p_{b} . n .
$$

Here $t-R$ is the usual retarded time and the $2 G \ln R \sum_{b=1}^{n} p_{b} . n$ takes into account the effect of the long range gravitational force on the gravitational wave as it travels from the scattering center to the detector. $G$ denotes the Newton's constant. We have used units in which the velocity of light $c$ has been set equal to 1 , - this is the unit we shall use throughout the paper. We also define the deviation of the metric $g_{\mu \nu}$ from flat metric via:

$$
h_{\mu \nu} \equiv\left(g_{\mu \nu}-\eta_{\mu \nu}\right) / 2, \quad e_{\mu \nu} \equiv h_{\mu \nu}-\frac{1}{2} \eta_{\mu \nu} \eta^{\rho \sigma} h_{\rho \sigma} .
$$

Let us first assume that the objects do not carry charge so that gravity is the only long range force acting on the objects at late and early time, although during the scattering

\footnotetext{
${ }^{1} \mathrm{~A}$ possible explanation of these logarithmic terms using asymptotic symmetries has been discussed recently [20, 21].
} 
they may undergo complicated interactions. Then at late and early retarded time, our result for the gravitational wave-form at the detector is given by:

$$
\begin{aligned}
& \text { Late time : } e^{\mu \nu}(t, R, \hat{n})=\frac{2 G}{R}\left[-\sum_{a=1}^{n} p_{a}^{\mu} p_{a}^{\nu} \frac{1}{n \cdot p_{a}}+\sum_{a=1}^{m} p_{a}^{\prime \mu} p_{a}^{\prime \nu} \frac{1}{n \cdot p_{a}^{\prime}}\right] \\
& -\frac{4 G^{2}}{R u}\left[\sum_{a=1}^{n} \sum_{\substack{b=1 \\
b \neq a}}^{n} \frac{p_{a} \cdot p_{b}}{\left\{\left(p_{a} \cdot p_{b}\right)^{2}-p_{a}^{2} p_{b}^{2}\right\}^{3 / 2}}\left\{\frac{3}{2} p_{a}^{2} p_{b}^{2}-\left(p_{a} \cdot p_{b}\right)^{2}\right\} \frac{n_{\rho} p_{a}^{\mu}}{n \cdot p_{a}}\left(p_{b}^{\rho} p_{a}^{\nu}-p_{b}^{\nu} p_{a}^{\rho}\right)\right. \\
& \left.-\sum_{b=1}^{n} p_{b} . n\left\{\sum_{a=1}^{n} \frac{1}{p_{a} \cdot n} p_{a}^{\mu} p_{a}^{\nu}-\sum_{a=1}^{m} \frac{1}{p_{a}^{\prime} \cdot n} p_{a}^{\prime \mu} p_{a}^{\prime \nu}\right\}\right]+\mathcal{O}\left(u^{-2}\right), \quad \text { as } u \rightarrow \infty
\end{aligned}
$$

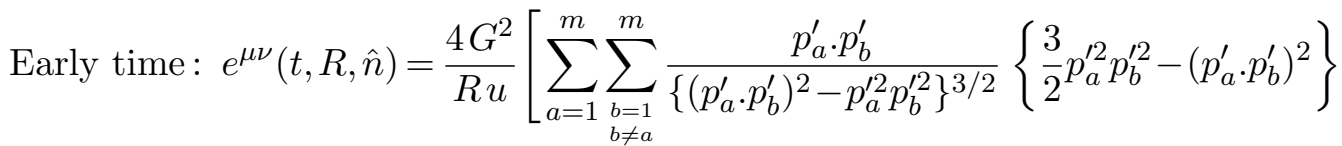

$$
\left.\times \frac{n_{\rho} p_{a}^{\prime \mu}}{n \cdot p_{a}^{\prime}}\left(p_{b}^{\prime \rho} p_{a}^{\prime \nu}-p_{b}^{\prime \nu} p_{a}^{\prime \rho}\right)\right]+\mathcal{O}\left(u^{-2}\right), \quad \text { as } u \rightarrow-\infty,
$$

where $\mathcal{O}\left(u^{-2}\right)$ includes terms of order $u^{-2} \ln |u|$. The term on the right hand side of the first line represents a constant jump in $h_{\mu \nu}$ during the passage of the gravitational wave, and is known as the memory effect [22-31]. This is related to the leading soft theorem [32]. The terms of order $1 / u$ are related to logarithmic corrections to the subleading soft theorem. These have been verified in various examples via explicit calculations [33-35]. The sum over $a$ in (1.4) also includes the contribution from finite frequency radiation emitted during the scattering. Different limits of the Fourier transforms of these results have been studied in section 2.3 of [18].

As already discussed in $[18,19]$, in case of decay $(m=1)$, if at most one of the final objects is massive and the rest are massless, including radiation, then the terms proportional to $1 / u$ in the expression for $e^{\mu \nu}$ cancel. This will be the case for binary black hole merger where the initial state is a single bound system, and the final state consists of a single massive black hole and gravitational radiation. Therefore absence of $1 / u$ tails in such decays can be taken as a test of general theory of relativity.

If the objects participating in the scattering process are charged, with the incoming objects carrying charges $q_{1}^{\prime}, \cdots, q_{m}^{\prime}$ and outgoing objects carrying charges $q_{1}, \cdots, q_{n}$, then there are further corrections to (1.4) due to long range electromagnetic forces between the incoming and the outgoing objects. These corrections have been given in (4.30).

A similar result can be given for the profile of the electromagnetic vector potential $a_{\mu}$ at the detector at late and early retarded time. The results are given in (4.23), (4.24).

Although these results are derived in this paper for the first time, they have been conjectured earlier from soft graviton theorem following the chain of arguments given at the beginning of this section. Emboldened by the success of these arguments, we describe in section 5 a new conjecture for terms of order $u^{-2} \ln |u|$ at late and early retarded time. These have been given in (5.7), (5.8) and (5.9). 


\section{Some useful results}

In this section we shall set up some notations and collect some mathematical results that will be used in our analysis. The derivation of these results can be found in appendix A.

We begin by setting up our notation for different Fourier transforms that we shall use in our analysis. We shall deal with functions of four variables $x \equiv(t, \vec{x}) \equiv\left(x^{0}, x^{1}, x^{2}, x^{3}\right)$ describing the space-time coordinates. Given any such function $F(x)$, we shall introduce the following different kinds of Fourier transforms:

$$
\begin{aligned}
\widehat{F}(k) & \equiv \int d^{4} x e^{-i k \cdot x} F(t, \vec{x}), \\
\bar{F}(t, \vec{k}) & \equiv \int d^{3} x e^{-i \vec{k} \cdot \vec{x}} F(t, \vec{x}), \\
\widetilde{F}(\omega, \vec{x}) & \equiv \int d t e^{i \omega t} F(t, \vec{x}) .
\end{aligned}
$$

The inverse relations are

$$
\begin{aligned}
& F(t, \vec{x})=\int \frac{d^{4} k}{(2 \pi)^{4}} e^{i k \cdot x} \widehat{F}(k), \\
& F(t, \vec{x})=\int \frac{d^{3} k}{(2 \pi)^{3}} e^{i \vec{k} \cdot \vec{x}} \bar{F}(t, \vec{k}), \\
& F(t, \vec{x})=\int \frac{d \omega}{2 \pi} e^{-i \omega t} \widetilde{F}(\omega, \vec{x}) .
\end{aligned}
$$

Note that we are using the convention $k \cdot x \equiv \eta_{\mu \nu} k^{\mu} x^{\nu}=-k^{0} x^{0}+\vec{k} \cdot \vec{x}$.

Let us consider a differential equation of the form:

$$
\square F(x)=-j(x), \quad \square \equiv \eta^{\alpha \beta} \partial_{\alpha} \partial_{\beta},
$$

where $j(x)$ is some given function. Then one can show that, at large distance $R$,

$$
\widetilde{F}(\omega, \vec{x}) \simeq \frac{1}{4 \pi R} e^{i \omega R} \widehat{j}(k), \quad R \equiv|\vec{x}|, \quad \hat{n} \equiv \frac{\vec{x}}{R}, \quad k \equiv \omega(1, \hat{n}),
$$

The derivation of this relation has been reviewed in appendix A.1.

We shall now give the results of Fourier transforms of some functions that are singular at the origin. Let $f(\omega)$ be some function that is analytic on the real axis with $f(0)=1$, and assume that it falls of sufficiently fast at large $|\omega|$ so as to render finite the various integrals that will appear below. Then we have the following results:

$$
\frac{1}{2 \pi} \int d \omega e^{-i \omega u} \frac{1}{\omega+i \epsilon} f(\omega)=-i H(u)+\mathcal{O}\left(e^{-u}\right),
$$

where $H$ is the Heaviside step function. Changing the sign of the $i \epsilon$ or using principal value only adds a constant to the expression, so that the jump in the function as we change $u$ 
from large negative to large positive value remains unchanged. We also have,

$$
\begin{aligned}
& \int \frac{d \omega}{2 \pi} e^{-i \omega u} \ln (\omega+i \epsilon) f(\omega) \rightarrow\left\{\begin{array}{l}
-\frac{1}{u} \text { for } u \rightarrow \infty \\
0 \text { for } u \rightarrow-\infty
\end{array}\right.
\end{aligned}
$$

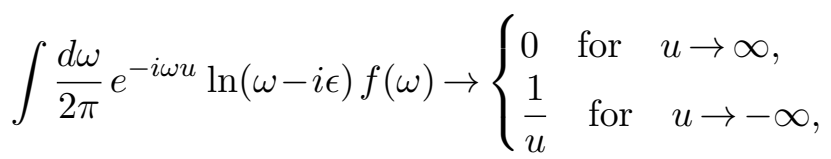

$$
\begin{aligned}
& \int \frac{d \omega}{2 \pi} e^{-i \omega u} \omega\{\ln (\omega+i \epsilon)\}^{2} f(\omega) \rightarrow\left\{\begin{array}{l}
-2 i u^{-2} \ln |u|+\mathcal{O}\left(u^{-2}\right) \\
0 \quad \text { for } \quad u \rightarrow-\infty,
\end{array} \text { for } u \rightarrow \infty,\right. \\
& \int \frac{d \omega}{2 \pi} e^{-i \omega u} \omega\{\ln (\omega-i \epsilon)\}^{2} f(\omega) \rightarrow\left\{\begin{array}{l}
0 \quad \text { for } \quad u \rightarrow \infty, \\
2 i u^{-2} \ln |u|+\mathcal{O}\left(u^{-2}\right) \text { for } u \rightarrow-\infty
\end{array}\right. \\
& \int \frac{d \omega}{2 \pi} e^{-i \omega u} \omega \ln (\omega+i \epsilon) \ln (\omega-i \epsilon) f(\omega) \rightarrow\left\{\begin{array}{l}
-i u^{-2} \ln |u|+\mathcal{O}\left(u^{-2}\right) \text { for } u \rightarrow \infty \\
i u^{-2} \ln |u|+\mathcal{O}\left(u^{-2}\right) \text { for } u \rightarrow-\infty
\end{array}\right.
\end{aligned}
$$

Eqs. (2.5)-(2.8) have been proved in appendix A.2.

\section{Proof of classical soft graviton theorem}

We consider a scattering event in asymptotically flat space-time in which $m$ objects carrying masses $\left\{m_{a}^{\prime}\right\}$, four velocities $\left\{v_{a}^{\prime}\right\}$ and four momenta $\left\{p_{a}^{\prime}=m_{a}^{\prime} v_{a}^{\prime}\right\}$ for $1 \leq a \leq m$ come close, undergo complicated interactions, and disperse as $n$ objects carrying masses $\left\{m_{a}\right\}$, four velocities $\left\{v_{a}\right\}$ and four momenta $\left\{p_{a}\right\}$ for $1 \leq a \leq n$. We do not assume that the interactions are weak, and they could involve exchange of energy and other quantum numbers, fusion and splitting. Our goal will be to compute the gravitational wave-form emitted during this scattering event at early and late retarded time. As discussed in section A.2, this is related to the behaviour of the Fourier transform of the wave-form in the low frequency limit.

Since we shall be interested in the long wavelength gravitational waves emitted by the system, we can represent the leading contribution to the energy momentum tensor of the incoming and outgoing objects by the energy momentum tensor of point particles, and include the effect of internal structure of the objects by adding subleading contributions involving higher derivative terms [37-45]. In fact, to the order at which we shall be working, it will be sufficient to keep just the leading term. For this reason, we shall henceforth refer to the incoming and outgoing objects as particles.

The strategy we shall follow will be to iteratively solve the coupled equations of motion of matter and gravity using Feynman diagram like techniques. This method has been widely used in recent years [46-50], most notably in [36, 51-53]. However the main difference between our approach and the earlier ones is in setting up the boundary conditions. In the usual approach we set the initial condition and evolve the system using the equations of motion, computing both the trajectories and the emitted radiation during this process. 


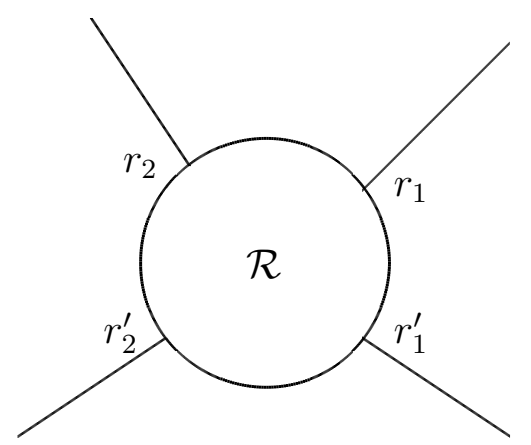

Figure 1. A scattering process in which the particles interact strongly inside the region $\mathcal{R}$ via some unspecified forces, but outside the region $\mathcal{R}$ the only force operative between the particles is the long range gravitational force.

In our approach we take the initial and final momenta as given, but allow the interactions during the scattering to be arbitrary. Therefore while solving the equations we need to evolve the initial particle trajectories forward in time and the final particle trajectories backward in time, and compute the net gravitational wave emitted during the scattering.

For simplicity, in this section we shall consider the situation where the particles are uncharged so that there are no long range electromagnetic interactions between the asymptotic particles. The effect of such interactions will be incorporated in section 4.4.

\subsection{General set-up}

We choose the origin of the space-time coordinate system to be somewhere within the region where the scattering takes place and denote by $\mathcal{R}$ a large but finite region of spacetime so that the non-trivial part of the scattering occurs within the region $\mathcal{R}$. In particular we shall choose $\mathcal{R}$ to be sufficiently large so that outside the region $\mathcal{R}$ the only interaction that exists between the particles is the long range gravitational interaction. This has been shown in figure 1 . We shall denote by $L$ the linear size of $\mathcal{R}$ and analyze gravitational radiation at retarded time $u$ for $|u| \gg L$.

We define:

$$
h_{\mu \nu}=\frac{1}{2}\left(g_{\mu \nu}-\eta_{\mu \nu}\right), \quad e_{\mu \nu}=h_{\mu \nu}-\frac{1}{2} \eta_{\mu \nu} \eta^{\rho \sigma} h_{\rho \sigma} \Leftrightarrow h_{\mu \nu}=e_{\mu \nu}-\frac{1}{2} \eta_{\mu \nu} \eta^{\rho \sigma} e_{\rho \sigma} .
$$

We denote by $X_{a}(\sigma)$ for $1 \leq a \leq n$ the outgoing particle trajectories parametrized by the proper time ${ }^{2} \sigma$ in the range $0 \leq \sigma<\infty$, with $\sigma=0$ labelling the point where the trajectory exits the region $\mathcal{R}$. Similarly $X_{a}^{\prime}(\sigma)$ for $1 \leq a \leq m$ will denote the incoming particle trajectories parametrized by the proper time $\sigma$ in the range $-\infty<\sigma \leq 0$, with $\sigma=0$ labelling the point where the trajectory enters the region $\mathcal{R}$. We now consider the

\footnotetext{
${ }^{2}$ More precisely, $\sigma$ is a parameter labelling the trajectory, that is set equal to the proper time after deriving the equations of motion.
} 
Einstein's action coupled to these particles:

$$
\begin{aligned}
S= & \frac{1}{16 \pi G} \int d^{4} x \sqrt{-\operatorname{det} g} R-\sum_{a=1}^{n} \int_{0}^{\infty} d \sigma m_{a}\left\{-g_{\mu \nu}(X(\sigma)) \frac{d X_{a}^{\mu}}{d \sigma} \frac{d X_{a}^{\nu}}{d \sigma}\right\}^{1 / 2} \\
& -\sum_{a=1}^{m} \int_{-\infty}^{0} d \sigma m_{a}^{\prime}\left\{-g_{\mu \nu}\left(X^{\prime}(\sigma)\right) \frac{d X_{a}^{\prime \mu}}{d \sigma} \frac{d X_{a}^{\prime \nu}}{d \sigma}\right\}^{1 / 2} .
\end{aligned}
$$

Note that we have included in the action the contribution only from part of the particle trajectories that lie outside the region $\mathcal{R}$. We shall argue later that this action is sufficient for determining the gravitational wave-form at late and early time. We now derive the equations of motion for $e_{\mu \nu}$ by extremizing the action (3.2) with respect to $e_{\mu \nu}$. This takes the form:

$$
\sqrt{-\operatorname{det} g}\left(R^{\mu \nu}-\frac{1}{2} g^{\rho \sigma} R_{\rho \sigma} g^{\mu \nu}\right)=8 \pi G T^{X \mu \nu},
$$

where,

$$
\begin{aligned}
T^{X \mu \nu} \equiv & \sum_{a=1}^{n} m_{a} \int_{0}^{\infty} d \sigma \delta^{(4)}\left(x-X_{a}(\sigma)\right) \frac{d X_{a}^{\mu}}{d \sigma} \frac{d X_{a}^{\nu}}{d \sigma} \\
& +\sum_{a=1}^{m} m_{a}^{\prime} \int_{-\infty}^{0} d \sigma \delta^{(4)}\left(x-X_{a}^{\prime}(\sigma)\right) \frac{d X_{a}^{\prime \mu}}{d \sigma} \frac{d X_{a}^{\prime \nu}}{d \sigma}
\end{aligned}
$$

Note the factor of $\sqrt{-\operatorname{det} g}$ and the raised indices on the left hand side of (3.3) - this makes the right hand side independent of the metric. After imposing the de Donder gauge:

$$
\eta^{\mu \nu} \partial_{\mu} h_{\nu \lambda}-\frac{1}{2} \partial_{\lambda}\left(\eta^{\rho \sigma} h_{\rho \sigma}\right)=0 \quad \Leftrightarrow \quad \eta^{\mu \nu} \partial_{\mu} e_{\nu \lambda}=0,
$$

and expanding the left hand side of (3.3) in power series in $h_{\mu \nu}$, we can express the equations of motion of the metric as:

$$
\eta^{\alpha \mu} \eta^{\beta \nu} \eta^{\rho \sigma} \partial_{\rho} \partial_{\sigma} e_{\alpha \beta}=-8 \pi G T^{\mu \nu}(x), \quad T^{\mu \nu} \equiv T^{X \mu \nu}+T^{h \mu \nu},
$$

where $T^{h \mu \nu}$ denotes the gravitational stress tensor, defined as what we obtain by taking all $e_{\alpha \beta}$ dependent terms on the left hand side of (3.3), except the terms linear in $e_{\alpha \beta}$, to the right hand side and dividing it by $8 \pi G$. In all subsequent equations, the indices will be raised and lowered by $\eta_{\mu \nu}$.

Our goal is to compute $e_{\mu \nu}(t, \vec{x})$ at a point far away from the scattering center. We shall label $\vec{x}$ as $R \hat{n}$ where $\hat{n}$ is a unit vector and $R \equiv|\vec{x}|$. It follows from (A.2) and (2.4) that the retarded solution to $(3.6)$ is given by $[15]^{3}$

$$
\tilde{e}_{\mu \nu}(\omega, R, \hat{n})=\frac{2 G}{R} e^{i \omega R} \widehat{T}_{\mu \nu}(k)+\mathcal{O}\left(R^{-2}\right),
$$

where

$$
\widehat{T}_{\mu \nu}(k) \equiv \int d^{4} x e^{-i k \cdot x} T_{\mu \nu}(x),
$$

${ }^{3}(3.7)$ can also be written as

$$
e_{\mu \nu}(t, R, \hat{n})=\frac{2 G}{R} \bar{T}_{\mu \nu}(t-R, \vec{k})+\mathcal{O}\left(R^{-2}\right) .
$$


is the Fourier transform of $T_{\mu \nu}(x)$ in all the variables and $k=\omega(1, \hat{n})$ as defined in (A.8). Therefore we need to compute $\widehat{T}_{\mu \nu}(k)$. Furthermore, it follows from the analysis of section A.2 that to extract the late and early time behaviour of $e_{\mu \nu}(t, \vec{x})$ we need to examine the non-analytic part of $\tilde{e}_{\mu \nu}(\omega, R, \hat{n})$ as a function of $\omega$ - in particular terms of order $1 / \omega$ and $\ln \omega$. For this, we can restrict the integration over $x$ in (3.8) to outside the region $\mathcal{R}$, since integration over a finite region of space-time will give an infrared finite contribution and cannot generate a singularity as $\omega \rightarrow 0$. This justifies the omission of the contribution to the action (3.2) from particle trajectories inside the region $\mathcal{R}$.

We shall compute $\widehat{T}_{\mu \nu}$ by solving the following equations iteratively:

$$
\begin{aligned}
T^{\mu \nu}(x)= & T^{X \mu \nu}(x)+T^{h \mu \nu}(x), \\
T^{X \mu \nu}(x) \equiv & \sum_{a=1}^{n} m_{a} \int_{0}^{\infty} d \sigma \delta^{(4)}\left(x-X_{a}(\sigma)\right) \frac{d X_{a}^{\mu}}{d \sigma} \frac{d X_{a}^{\nu}}{d \sigma} \\
& +\sum_{a=1}^{m} m_{a}^{\prime} \int_{-\infty}^{0} d \sigma \delta^{(4)}\left(x-X_{a}^{\prime}(\sigma)\right) \frac{d X_{a}^{\prime \mu}}{d \sigma} \frac{d X_{a}^{\prime \nu}}{d \sigma}, \\
\square e_{\mu \nu}= & -8 \pi G T_{\mu \nu} \equiv-8 \pi G \eta_{\mu \alpha} \eta_{\nu \beta} T^{\alpha \beta}, \\
\frac{d^{2} X_{a}^{\mu}}{d \sigma^{2}}= & -\Gamma_{\nu \rho}^{\mu}(X(\sigma)) \frac{d X_{a}^{\nu}}{d \sigma} \frac{d X_{a}^{\rho}}{d \sigma}, \quad \frac{d^{2} X_{a}^{\prime \mu}}{d \sigma^{2}}=-\Gamma_{\nu \rho}^{\mu}\left(X^{\prime}(\sigma)\right) \frac{d X_{a}^{\prime \nu}}{d \sigma} \frac{d X_{a}^{\prime \rho}}{d \sigma}
\end{aligned}
$$

with boundary conditions:

$$
\begin{aligned}
X_{a}^{\mu}(\sigma=0)=r_{a}^{\mu}, & \lim _{\sigma \rightarrow \infty} \frac{d X_{a}^{\mu}}{d \sigma}=v_{a}^{\mu}=\frac{1}{m_{a}} p_{a}^{\mu}, \\
X_{a}^{\prime \mu}(\sigma=0)=r_{a}^{\prime \mu}, & \lim _{\sigma \rightarrow-\infty} \frac{d X_{a}^{\prime \mu}}{d \sigma}=v_{a}^{\prime \mu}=\frac{1}{m_{a}^{\prime}} p_{a}^{\prime \mu} .
\end{aligned}
$$

Here $\Gamma_{\nu \rho}^{\mu}$ denotes the Christoffel symbol constructed from the metric $\eta_{\mu \nu}+2 h_{\mu \nu} \cdot r_{a}$ denotes the point where the trajectory of the $a$-th outgoing particle intersects the boundary of $\mathcal{R}$ and $r_{a}^{\prime}$ denotes the point where the trajectory of the $a$-th incoming particle intersects the boundary of $\mathcal{R} . T^{h}$ is the stress tensor of gravity, as defined below (3.6). $h_{\mu \nu}$ and hence $e_{\mu \nu}$ is required to satisfy retarded boundary condition. The starting solution for the iteration is taken to be

$$
e_{\mu \nu}=0, \quad X_{a}^{\mu}(\sigma)=r_{a}^{\mu}+v_{a}^{\mu} \sigma=r_{a}^{\mu}+\frac{1}{m_{a}} p_{a}^{\mu} \sigma, \quad X_{a}^{\prime \mu}(\sigma)=r_{a}^{\prime \mu}+v_{a}^{\prime \mu} \sigma=r_{a}^{\prime \mu}+\frac{1}{m_{a}^{\prime}} p_{a}^{\prime \mu} \sigma .
$$

We can give a uniform treatment of the incoming and the outgoing particles by defining:

$$
\begin{gathered}
X_{a+n}^{\mu}(\sigma)=X_{a}^{\prime \mu}(-\sigma), \quad m_{a+n}=m_{a}^{\prime}, \quad v_{a+n}^{\mu}=-v_{a}^{\prime \mu}, \quad r_{a+n}^{\mu}=r_{a}^{\prime \mu}, \quad p_{a+n}^{\mu}=-p_{a}^{\prime \mu}, \\
\text { for } 1 \leq a \leq m .
\end{gathered}
$$

In this case we can express (3.9) and (3.10) as:

$$
\begin{aligned}
T^{\mu \nu}(x) & =T^{X \mu \nu}(x)+T^{h \mu \nu}(x), \\
T^{X \mu \nu}(x) & \equiv \sum_{a=1}^{m+n} m_{a} \int_{0}^{\infty} d \sigma \delta^{(4)}\left(x-X_{a}(\sigma)\right) \frac{d X_{a}^{\mu}}{d \sigma} \frac{d X_{a}^{\nu}}{d \sigma} \\
\square e_{\mu \nu} & =-8 \pi G T_{\mu \nu}, \quad \frac{d^{2} X_{a}^{\mu}}{d \sigma^{2}}=-\Gamma_{\nu \rho}^{\mu}(X(\sigma)) \frac{d X_{a}^{\nu}}{d \sigma} \frac{d X_{a}^{\rho}}{d \sigma}, \quad \text { for } 1 \leq a \leq m+n,
\end{aligned}
$$


and

$$
X_{a}^{\mu}(\sigma=0)=r_{a}^{\mu}, \quad \lim _{\sigma \rightarrow \infty} \frac{d X_{a}^{\mu}}{d \sigma}=v_{a}^{\mu}=\frac{1}{m_{a}} p_{a}^{\mu}, \quad \text { for } 1 \leq a \leq m+n .
$$

Also the starting solution (3.11) for iteration may be written as

$$
e_{\mu \nu}=0, \quad X_{a}^{\mu}(\sigma)=r_{a}^{\mu}+v_{a}^{\mu} \sigma=r_{a}^{\mu}+\frac{1}{m_{a}} p_{a}^{\mu} \sigma, \quad \text { for } 1 \leq a \leq m+n .
$$

From now on we shall follow this convention, with the understanding that the sum over $a$ always runs from 1 to $(m+n)$ unless stated otherwise.

\subsection{Leading order contribution}

At the leading order in the expansion in powers of $G, T^{h \mu \nu}$ vanishes, and we have:

$$
\begin{aligned}
\widehat{T}^{\mu \nu}(k)=\widehat{T}^{X \mu \nu}(k) & =\int d^{4} x e^{-i k \cdot x} \sum_{a=1}^{m+n} m_{a} \int_{0}^{\infty} d \sigma \delta^{(4)}\left(x-X_{a}(\sigma)\right) \frac{d X_{a}^{\mu}}{d \sigma} \frac{d X_{a}^{\nu}}{d \sigma} \\
& =\sum_{a=1}^{m+n} m_{a} \int_{0}^{\infty} d \sigma e^{-i k \cdot X(\sigma)} \frac{d X_{a}^{\mu}}{d \sigma} \frac{d X_{a}^{\nu}}{d \sigma}
\end{aligned}
$$

where, as mentioned earlier, we have restricted the region of integration over $x$ to outside the region $\mathcal{R}$. Using the leading order solution (3.15) we get

$$
\begin{aligned}
\widehat{T}^{\mu \nu}(k) & =\sum_{a=1}^{m+n} m_{a} \int_{0}^{\infty} d \sigma e^{-i k \cdot\left(v_{a} \sigma+r_{a}\right)} v_{a}^{\mu} v_{a}^{\nu}=\sum_{a=1}^{m+n} m_{a} \frac{1}{i\left(k \cdot v_{a}-i \epsilon\right)} e^{-i k \cdot r_{a}} v_{a}^{\mu} v_{a}^{\nu} \\
& =\sum_{a=1}^{m+n} p_{a}^{\mu} p_{a}^{\nu} e^{-i k \cdot r_{a}} \frac{1}{i\left(k \cdot p_{a}-i \epsilon\right)} .
\end{aligned}
$$

The $i \epsilon$ prescription is obtained by noting that addition of a small negative imaginary part to $k \cdot v_{a}$ makes the $\sigma$ integrals convergent. Therefore the poles must be in the upper half $k . v_{a}$ plane. Note that the $i \epsilon$ prescription is independent of whether $k \cdot p_{a}$ is positive or negative, i.e. whether $p_{a}$ represents an ingoing or an outgoing momentum, since after the redefinition (3.12), $\sigma$ always runs from 0 to $\infty$. A Feynman diagram like representation of (3.17) in the spirit of [36, 51-53] can be found in figure 2 .

Since we are looking for terms that are singular at $\omega \rightarrow 0$, i.e. $k^{\mu} \rightarrow 0$, we can replace the $e^{-i k . r_{a}}$ factors by 1 . This gives the leading soft factor associated with the memory effect.

\subsection{First order correction to the gravitational field}

We now turn to the next order contribution. We first solve for $e_{\mu \nu}$ satisfying the third equation in (3.13) as

$$
\begin{aligned}
\widehat{e}_{\mu \nu}(k) & =-8 \pi G G_{r}(k) \widehat{T}_{\mu \nu}(k)=-8 \pi G \sum_{a=1}^{m+n} p_{a \mu} p_{a \nu} e^{-i k \cdot r_{a}} G_{r}(k) \frac{1}{i\left(k \cdot p_{a}-i \epsilon\right)}, \\
G_{r}(k) & \equiv \frac{1}{\left(k^{0}+i \epsilon\right)^{2}-\vec{k}^{2}} .
\end{aligned}
$$




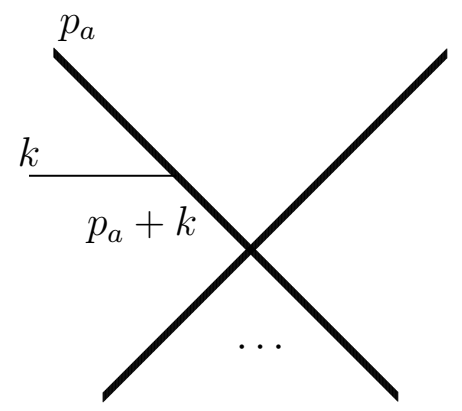

Figure 2. A Feynman diagram like representation of (3.17), with the external thin line carrying momentum $k$ labelling the argument of $\widehat{T}^{\mu \nu}(k)$, the external thick lines representing the incoming and the outgoing particles and the internal propagator carrying momentum $p_{a}+k$ representing the $1 /\left\{i\left(p_{a} \cdot k-i \epsilon\right)\right\}$ factor. All momenta are labelled as outgoing, so an incoming particle is represented with negative $p_{a}^{0}$. The vertex where the lines representing incoming and outgoing particles meet represent the interaction region $R$ in figure 1 . The vertex where the external thin line representing $\widehat{T}^{\mu \nu}(k)$ meets the $a$-th thick line carries the $p_{a}^{\mu} p_{a}^{\nu}$ factor in (3.17).

One comment is in order here. The expression (3.16) for $\widehat{T}^{\mu \nu}(k)$, which we are using in (3.18), ignores the contribution from the region of integration $\mathcal{R}$. This was justified earlier since we were computing the singular part of $\widehat{T}_{\mu \nu}$. However, now we need the contribution to $\widehat{e}_{\mu \nu}$ from the full $\widehat{T}_{\mu \nu}$ since our goal will be to use this to compute $T_{\mu \nu}^{h}$, and also to compute the corrections to the particle trajectories, which, in turn, give corrections to $T_{\mu \nu}^{X}$. Once we compute these, we use (3.7) to compute $\widetilde{e}_{\mu \nu}$. At this stage, we can again restrict the integration region to outside $\mathcal{R}$ while taking the Fourier transform to compute the corrected $\widehat{T}_{\mu \nu}$. To address this issue, we first analyze the possible correction $\delta T_{\mu \nu}^{X}$ to $T_{\mu \nu}^{X}$ due to gravitational fields generated from inside $\mathcal{R}$. Since in four space-time dimensions the retarded Green's function has support on the future light-cone, the field sourced by energy momentum tensor inside $\mathcal{R}$ will have support on the future light-cone emerging from points inside $\mathcal{R}$. These intersect the time-like trajectories of the outgoing (or incoming) particles emerging from $\mathcal{R}$ only within a distance of order $L$ - the size of $\mathcal{R}$. Therefore $\delta T_{\mu \nu}^{X}$ is affected only in this region. Since integration over this region will not produce a singular contribution to $\widehat{T}_{\mu \nu}^{X}(k)$ in the $\omega \rightarrow 0$ limit, this effect may be ignored. However the gravitational field produced from the sources inside $\mathcal{R}$ could give significant contribution to $T^{h}$, since we are not assuming the interactions inside $\mathcal{R}$ to be weak. We take this into account by regarding the contribution to $\widehat{e}_{\mu \nu}(k)=-8 \pi G G_{r}(k) \widehat{T}_{\mu \nu}(k)$ from inside the region $\mathcal{R}$ as a flux of finite wavelength gravitational waves produced by $T_{\mu \nu}(x)$ inside $\mathcal{R}$, and include this in the sum over $a$. Therefore the outgoing momenta $\left\{p_{a}\right\}$ not only will include finite mass particles, but also the finite wave-length 'massless gravitons' emitted during the scattering process.

Using (3.18) we can calculate, at the next order,

$$
\begin{aligned}
& e_{\mu \nu}^{(b)}(x)=-8 \pi G \int \frac{d^{4} \ell}{(2 \pi)^{4}} e^{i \ell \cdot\left(x-r_{b}\right)} G_{r}(\ell) p_{b \mu} p_{b \nu} \frac{1}{i\left(\ell \cdot p_{b}-i \epsilon\right)}, \\
& h_{\mu \nu}^{(b)}(x)=-8 \pi G \int \frac{d^{4} \ell}{(2 \pi)^{4}} e^{i \ell \cdot\left(x-r_{b}\right)} G_{r}(\ell)\left\{p_{b \mu} p_{b \nu}-\frac{1}{2} p_{b}^{2} \eta_{\mu \nu}\right\} \frac{1}{i\left(\ell \cdot p_{b}-i \epsilon\right)},
\end{aligned}
$$


where $e_{\mu \nu}^{(b)}$ is the gravitational field due to the $b$-th particle. This gives

$$
\begin{aligned}
\Gamma_{\nu \rho}^{(b) \mu}(x)= & \eta^{\mu \alpha}\left\{\partial_{\nu} h_{\alpha \rho}^{(b)}+\partial_{\rho} h_{\alpha \nu}^{(b)}-\partial_{\alpha} h_{\nu \rho}^{(b)}\right\} \\
= & -8 \pi G \int \frac{d^{4} \ell}{(2 \pi)^{4}} e^{i \ell .\left(x-r_{b}\right)} G_{r}(\ell) \frac{1}{\left(\ell \cdot p_{b}-i \epsilon\right)}\left[\left\{\ell_{\nu} p_{b}^{\mu} p_{b \rho}+\ell_{\rho} p_{b}^{\mu} p_{b \nu}-\ell^{\mu} p_{b \nu} p_{b \rho}\right\}\right. \\
& \left.-\frac{1}{2} p_{b}^{2}\left\{\ell_{\nu} \delta_{\rho}^{\mu}+\ell_{\rho} \delta_{\nu}^{\mu}-\ell^{\mu} \eta_{\nu \rho}\right\}\right] .
\end{aligned}
$$

These results will be used for two purposes. We shall substitute (3.20) into the last equation in (3.13) to compute the correction to the outgoing particle trajectories and hence to $T_{\mu \nu}^{X}$. We shall also use (3.19) to compute the leading contribution to $T_{\mu \nu}^{h}$.

Note that $e_{\mu \nu}^{(b)}(x)$ given in (3.19) satisfies:

$$
\partial_{\mu} e^{\mu \nu}=\sum_{b=1}^{m+n} \partial_{\mu} e^{(b) \mu \nu}(x)=-8 \pi G \sum_{b=1}^{m+n} \int \frac{d^{4} \ell}{(2 \pi)^{4}} e^{i \ell .\left(x-r_{b}\right)} G_{r}(\ell) p_{b}^{\nu} .
$$

As long as we restrict the integration range of $\ell$ to values for which $\ell .\left(r_{c}-r_{a}\right)$ is small for every pair $a, c$, we can take $e^{-i \ell \cdot r_{b}}$ to be approximately independent of $b$, and the right hand side of (3.21) vanishes due to momentum conservation law $\sum_{b=1}^{m+n} p_{b}^{\mu}=0$. Therefore $e^{\mu \nu}$ at this order satisfies the de Donder gauge condition:

$$
\partial^{\mu} e_{\mu \nu}=0
$$

At the next order there is apparent violation of this condition due to the $\ell . r_{b}$ factors coming from the expansion of the exponential factor. This can be compensated by some boundary terms on $\partial \mathcal{R}$ coming from integration inside the region $\mathcal{R}$ [16], but since these terms will not contribute to the singular terms that are of interest to us, we shall ignore them.

In the next two subsections we shall compute the correction to $\widehat{T}^{X}$ and $\widehat{T}^{h}$ using these results. It is also possible to argue that in order to calculate the logarithmic terms of interest, we can stop at this order. The natural dimensionless expansion parameter is $G M \omega$ where $M$ denotes the typical energy of the incoming / outgoing particles. Since the leading term (3.17) is of order $1 / \omega$, the subleading corrections that we shall compute will be of order $\omega^{0}$ multiplied by powers of $\ln \omega$. Higher order terms will involve higher powers of $\omega$ and will not be needed for our analysis.

\subsection{Subleading contribution to the matter stress tensor}

We begin by computing correction to the particle trajectory (3.15). Let $Y_{a}^{\mu}$ denote the correction:

$$
X_{a}^{\mu}(\sigma)=v_{a}^{\mu} \sigma+r_{a}^{\mu}+Y_{a}^{\mu}(\sigma) .
$$

Then $Y_{a}^{\mu}$ satisfies the differential equation and boundary conditions:

$$
\frac{d^{2} Y_{a}^{\mu}}{d \sigma^{2}}=-\Gamma_{\nu \rho}^{\mu}\left(v_{a} \sigma+r_{a}\right) v_{a}^{\nu} v_{a}^{\rho}, \quad Y_{a}^{\mu} \rightarrow 0 \text { as } \sigma \rightarrow 0, \quad \frac{d Y_{a}^{\mu}}{d \sigma} \rightarrow 0 \text { as } \sigma \rightarrow \infty
$$


where $^{4}$

$$
\Gamma_{\nu \rho}^{\mu}=\sum_{\substack{b=1 \\ b \neq a}}^{m+n} \Gamma_{\nu \rho}^{(b) \mu},
$$

captures the effect of the gravitational field produced by all particles other than $a$. Some of these terms must vanish, e.g. the gravitational field produced by an outgoing particle should not affect an incoming particle. This however will follow automatically from the equations that we shall derive, and need not be imposed externally. Integrating (3.24) we get

$$
\frac{d Y_{a}^{\mu}(\sigma)}{d \sigma}=\int_{\sigma}^{\infty} d \sigma^{\prime} \Gamma_{\nu \rho}^{\mu}\left(v_{a} \sigma^{\prime}+r_{a}\right) v_{a}^{\nu} v_{a}^{\rho}
$$

and

$$
Y_{a}^{\mu}(\sigma)=\int_{0}^{\sigma} d \sigma^{\prime} \int_{\sigma^{\prime}}^{\infty} d \sigma^{\prime \prime} \Gamma_{\nu \rho}^{\mu}\left(v_{a} \sigma^{\prime \prime}+r_{a}\right) v_{a}^{\nu} v_{a}^{\rho} .
$$

Substituting (3.23) into (3.16) we get $\widehat{T}^{X \mu \nu}$ to subleading order:

$$
\begin{aligned}
\widehat{T}^{X \mu \nu}(k) & =\sum_{a=1}^{m+n} m_{a} \int_{0}^{\infty} d \sigma e^{-i k .\left(v_{a} \sigma+r_{a}\right)}\left\{1-i k . Y_{a}(\sigma)\right\}\left\{v_{a}^{\mu}+\frac{d Y_{a}^{\mu}}{d \sigma}\right\}\left\{v_{a}^{\nu}+\frac{d Y_{a}^{\nu}}{d \sigma}\right\} \\
& =\sum_{a=1}^{m+n} m_{a} \int_{0}^{\infty} d \sigma e^{-i k .\left(v_{a} \sigma+r_{a}\right)}\left[v_{a}^{\mu} v_{a}^{\nu}-i k . Y_{a}(\sigma) v_{a}^{\mu} v_{a}^{\nu}+\frac{d Y_{a}^{\mu}}{d \sigma} v_{a}^{\nu}+v_{a}^{\mu} \frac{d Y_{a}^{\nu}}{d \sigma}\right] .
\end{aligned}
$$

Using (3.26), (3.27), we can express this as,

$$
\begin{aligned}
\widehat{T}^{X \mu \nu}(k)= & \sum_{a=1}^{m+n} m_{a} \int_{0}^{\infty} d \sigma e^{-i k .\left(v_{a} \sigma+r_{a}\right)} \\
& \times\left[v_{a}^{\mu} v_{a}^{\nu}-i k_{\rho} \int_{0}^{\sigma} d \sigma^{\prime} \int_{\sigma^{\prime}}^{\infty} d \sigma^{\prime \prime} \Gamma_{\alpha \beta}^{\rho}\left(v_{a} \sigma^{\prime \prime}+r_{a}\right) v_{a}^{\alpha} v_{a}^{\beta} v_{a}^{\nu} v_{a}^{\mu}\right. \\
& \left.\quad+\int_{\sigma}^{\infty} d \sigma^{\prime} \Gamma_{\alpha \beta}^{\mu}\left(v_{a} \sigma^{\prime}+r_{a}\right) v_{a}^{\alpha} v_{a}^{\beta} v_{a}^{\nu}+\int_{\sigma}^{\infty} d \sigma^{\prime} \Gamma_{\alpha \beta}^{\nu}\left(v_{a} \sigma^{\prime}+r_{a}\right) v_{a}^{\alpha} v_{a}^{\beta} v_{a}^{\mu}\right] .
\end{aligned}
$$

Substituting (3.20) and (3.25) into (3.29), and dropping the leading term given in (3.17), we get the first order correction to $\widehat{T}^{X}$ :

$$
\begin{gathered}
\Delta \widehat{T}^{X \mu \nu}(k)=-8 \pi G \sum_{a=1}^{m+n} \sum_{b \neq a} m_{a} \int \frac{d^{4} \ell}{(2 \pi)^{4}} \frac{1}{\ell \cdot p_{b}-i \epsilon} G_{r}(\ell)\left[\int_{0}^{\infty} d \sigma \int_{0}^{\sigma} d \sigma^{\prime} \int_{\sigma^{\prime}}^{\infty} d \sigma^{\prime \prime}\right. \\
e^{-i k \cdot v_{a} \sigma} e^{i \ell \cdot v_{a} \sigma^{\prime \prime}}\left\{-i v_{a} \cdot p_{b}\left(2 k \cdot p_{b} v_{a} \cdot \ell-k \cdot \ell v_{a} \cdot p_{b}\right)+\frac{i}{2} p_{b}^{2}\left(2 k \cdot v_{a} v_{a} \cdot \ell-k \cdot \ell v_{a}^{2}\right)\right\} v_{a}^{\nu} v_{a}^{\mu} \\
\quad+\int_{0}^{\infty} d \sigma \int_{\sigma}^{\infty} d \sigma^{\prime} e^{-i k \cdot v_{a} \sigma} e^{i \ell \cdot v_{a} \sigma^{\prime}} \\
\left\{2 \ell \cdot v_{a} v_{a} \cdot p_{b}\left(v_{a}^{\nu} p_{b}^{\mu}+v_{a}^{\mu} p_{b}^{\nu}\right)-\left(v_{a} \cdot p_{b}\right)^{2}\left(\ell^{\mu} v_{a}^{\nu}+\ell^{\nu} v_{a}^{\mu}\right)\right. \\
\left.\left.-2 \ell \cdot v_{a} p_{b}^{2} v_{a}^{\mu} v_{a}^{\nu}+\frac{1}{2} v_{a}^{2} p_{b}^{2}\left(\ell^{\mu} v_{a}^{\nu}+\ell^{\nu} v_{a}^{\mu}\right)\right\}\right] e^{-i k \cdot r_{a}-i \ell \cdot\left(r_{b}-r_{a}\right)}
\end{gathered}
$$

\footnotetext{
${ }^{4}$ The self-force effects [54] will not be important at this order.
} 
After carrying out the integrations over $\sigma, \sigma^{\prime}, \sigma^{\prime \prime}$, and using $p_{a}^{\mu}=m_{a} v_{a}^{\mu}$, we get

$$
\begin{aligned}
& \Delta \widehat{T}^{X \mu \nu}(k)=-8 \pi G \sum_{a=1}^{m+n} \sum_{b \neq a} \int \frac{d^{4} \ell}{(2 \pi)^{4}} \frac{1}{\ell \cdot p_{b}-i \epsilon} G_{r}(\ell) e^{-i k \cdot r_{a}-i \ell \cdot\left(r_{b}-r_{a}\right)} \\
& {\left[\left(2 p_{a} \cdot p_{b} k \cdot p_{b} p_{a} \cdot \ell-k \cdot \ell\left(p_{a} \cdot p_{b}\right)^{2}-p_{b}^{2} p_{a} \cdot k p_{a} \cdot \ell+\frac{1}{2} k \cdot \ell p_{a}^{2} p_{b}^{2}\right) p_{a}^{\nu} p_{a}^{\mu} \frac{1}{\ell \cdot p_{a}} \frac{1}{k \cdot p_{a}} \frac{1}{(\ell-k) \cdot p_{a}}\right.} \\
& -\left\{2 p_{a} \cdot p_{b} \ell \cdot p_{a}\left(p_{a}^{\nu} p_{b}^{\mu}+p_{a}^{\mu} p_{b}^{\nu}\right)-\left(p_{a} \cdot p_{b}\right)^{2}\left(\ell^{\mu} p_{a}^{\nu}+\ell^{\nu} p_{a}^{\mu}\right)-2 p_{b}^{2} \ell \cdot p_{a} p_{a}^{\mu} p_{a}^{\nu}\right. \\
& \left.\left.+\frac{1}{2} p_{a}^{2} p_{b}^{2}\left(\ell^{\mu} p_{a}^{\nu}+\ell^{\nu} p_{a}^{\mu}\right)\right\} \times \frac{1}{\ell \cdot p_{a}} \frac{1}{(\ell-k) \cdot p_{a}}\right] .
\end{aligned}
$$

For $\left|r_{a}^{\mu}-r_{b}^{\mu}\right| \sim L$, the ultraviolet divergence in the integration over $\ell$ is cut-off at $L^{-1}$ due to the oscillatory phase factor $e^{-i \ell .\left(r_{b}-r_{a}\right)}$. This contribution can be interpreted as the effect of first order correction to figure 2, induced by the correction to the trajectory of the $a$-th particle due to the gravitational field of the other particles.

In order to evaluate the integral, we need to determine the $i \epsilon$ prescription for the poles in (3.31). The $i \epsilon$ prescription for the $1 / \ell . p_{b}$ term has already been determined before. Similarly, since the $1 / \ell \cdot p_{a}$ factor comes from an integral in (3.30) of the form $\int_{\sigma^{\prime}}^{\infty} d \sigma^{\prime \prime} e^{i \ell \cdot v_{a} \sigma^{\prime \prime}}$ or $\int_{\sigma}^{\infty} d \sigma^{\prime} e^{i \ell \cdot v_{a} \sigma^{\prime}}$, the $i \epsilon$ prescription will be to replace $1 / \ell \cdot p_{a}$ by $1 /\left(\ell \cdot p_{a}+i \epsilon\right)$. The $1 / k \cdot p_{a}$ factor comes from an integral of the form $\int_{0}^{\infty} d \sigma e^{-i k \cdot v_{a} \sigma}$, and the $i \epsilon$ prescription will be to replace $1 / k \cdot p_{a}$ by $1 /\left(k \cdot p_{a}-i \epsilon\right)$. Finally, the $1 /(\ell-k) \cdot p_{a}$ factor in (3.31) arises from an integral of the form $\int_{0}^{\infty} d \sigma e^{i(\ell-k) \cdot v_{a} \sigma}$, and the correct $i \epsilon$ prescription for this term is $1 /\left((\ell-k) \cdot p_{a}+i \epsilon\right)$. Therefore, (3.31) should be written as

$$
\begin{aligned}
& \Delta \widehat{T}^{X \mu \nu}(k)=-8 \pi G \sum_{a=1}^{m+n} \sum_{b \neq a} \int \frac{d^{4} \ell}{(2 \pi)^{4}} \frac{1}{\ell \cdot p_{b}-i \epsilon} G_{r}(\ell) e^{-i k \cdot r_{a}-i \ell \cdot\left(r_{b}-r_{a}\right)} \\
& {\left[\left(2 p_{a} \cdot p_{b} k \cdot p_{b} p_{a} \cdot \ell-k \cdot \ell\left(p_{a} \cdot p_{b}\right)^{2}-p_{b}^{2} p_{a} \cdot k p_{a} \cdot \ell+\frac{1}{2} k \cdot \ell p_{a}^{2} p_{b}^{2}\right) p_{a}^{\nu} p_{a}^{\mu}\right.} \\
& \times \frac{1}{\ell \cdot p_{a}+i \epsilon} \frac{1}{k \cdot p_{a}-i \epsilon} \frac{1}{(\ell-k) \cdot p_{a}+i \epsilon} \\
& -\left\{2 p_{a} \cdot p_{b} \ell \cdot p_{a}\left(p_{a}^{\nu} p_{b}^{\mu}+p_{a}^{\mu} p_{b}^{\nu}\right)-\left(p_{a} \cdot p_{b}\right)^{2}\left(\ell^{\mu} p_{a}^{\nu}+\ell^{\nu} p_{a}^{\mu}\right)-2 p_{b}^{2} \ell \cdot p_{a} p_{a}^{\mu} p_{a}^{\nu}+\frac{1}{2} p_{a}^{2} p_{b}^{2}\left(\ell^{\mu} p_{a}^{\nu}+\ell^{\nu} p_{a}^{\mu}\right)\right\} \\
& \left.\quad \times \frac{1}{\ell \cdot p_{a}+i \epsilon} \frac{1}{(\ell-k) \cdot p_{a}+i \epsilon}\right] .
\end{aligned}
$$

Since we are interested in the singular term proportional to $\ln \omega$, we can simplify the analysis of the integral as follows. Since the expression is Lorentz covariant, we could evaluate it in a special frame in which $p_{a}$ and $p_{b}$ have only third component of spatial momenta. Let us denote by $\ell_{\perp}=\left(\ell^{1}, \ell^{2}\right)$ the transverse component of $\ell$. Now since $p_{a} . \ell$ and $p_{b} \cdot \ell$ are both linear in $\ell^{0}$ and $\ell^{3}$, we can use $p_{a} \cdot \ell$ and $p_{b} \cdot \ell$ as independent variables instead of $\ell^{0}$ and $\ell^{3}$. Then, if we ignore the poles of $G_{r}(\ell)$, we see that we have one pole in the $p_{b} . \ell$ plane and two poles on the same side of the real axis in the $p_{a} \cdot \ell$ plane. Therefore 
we can deform the $p_{a} \cdot \ell$ and $p_{b} . \ell$ integration contours away from the poles. However due to the presence of the $G_{r}(\ell)$ factor there are also poles at

$$
\left(\ell^{0}+i \epsilon+\ell^{3}\right)\left(\ell^{0}+i \epsilon-\ell^{3}\right)=\ell_{\perp}^{2} .
$$

Therefore, for small but fixed $\ell_{\perp}$, if we deform the $\left(\ell^{0}+\ell^{3}\right)$ contour to a distance of order $\left|\ell_{\perp}\right|$ away from the origin, a pole will approach the origin within a distance of order $\left|\ell_{\perp}\right|$ in the complex $\left(\ell^{0}-\ell^{3}\right)$ plane. The integration contour could then be pinched between this pole and one of the poles of the $\left(\ell \cdot p_{a}+i \epsilon\right)^{-1}\left\{(\ell-k) \cdot p_{a}+i \epsilon\right\}^{-1}\left(\ell \cdot p_{b}-i \epsilon\right)^{-1}$ factor. However it is clear that in the complex $\ell^{0}$ and complex $\ell^{3}$ plane, the integration contour can be deformed so that the contour maintains a minimum distance of order $\left|\ell_{\perp}\right|$ from all the poles, which themselves are situated within a distance of order $\left|\ell_{\perp}\right|$ of the origin. This shows that while estimating the integrand to examine possible sources of singularity of the integral, we can take all the components of $\ell$ to be of order $\ell_{\perp}$ and need not worry about the regions where one or more components are smaller than the others. Since for $\ell^{\mu} \sim \ell_{\perp}$ the integration measure gives a factor of $\left|\ell_{\perp}\right|^{4}$, we see that in order to get a logarithmic correction, the integrand must be of order $\left|\ell_{\perp}\right|^{-4}$.

We now note that in both terms the integrand of (3.32) grow as $\left|\ell_{\perp}\right|^{-3}$ for $\left|\ell^{\mu}\right| \sim$ $\left|\ell_{\perp}\right| \ll \omega$ and therefore there are no logarithmic corrections from this region. For $\mid r_{b}^{\mu}-$ $\left.r_{a}^{\mu}\right|^{-1} \sim L^{-1} \gg\left|\ell^{\mu}\right| \gg \omega$ we can replace $(k-\ell) \cdot p_{a}$ by $-\ell \cdot p_{a}$, and drop the $e^{-i \ell \cdot\left(r_{b}-r_{a}\right)}$ factor. In this case the integrand is of order $\left|\ell_{\perp}\right|^{-4}$ and the integral could have logarithmic contributions. To compute this, we note that in this region of integration the integral may be approximated as

$$
\begin{aligned}
& \Delta \widehat{T}^{X \mu \nu}(k) \simeq-8 \pi G \sum_{a} \sum_{b \neq a} \int \frac{d^{4} \ell}{(2 \pi)^{4}} \frac{1}{\ell \cdot p_{b}-i \epsilon} G_{r}(\ell) e^{-i k \cdot r_{a}} \\
& {\left[\left(2 p_{a} \cdot p_{b} k \cdot p_{b} p_{a} \cdot \ell-k \cdot \ell\left(p_{a} \cdot p_{b}\right)^{2}-p_{b}^{2} p_{a} \cdot k p_{a} \cdot \ell+\frac{1}{2} k \cdot \ell p_{a}^{2} p_{b}^{2}\right) p_{a}^{\nu} p_{a}^{\mu} \frac{1}{\left(\ell \cdot p_{a}+i \epsilon\right)^{2}} \frac{1}{k \cdot p_{a}-i \epsilon}\right.} \\
& -\left\{2 p_{a} \cdot p_{b} \ell \cdot p_{a}\left(p_{a}^{\nu} p_{b}^{\mu}+p_{a}^{\mu} p_{b}^{\nu}\right)-\left(p_{a} \cdot p_{b}\right)^{2}\left(\ell^{\mu} p_{a}^{\nu}+\ell^{\nu} p_{a}^{\mu}\right)-2 p_{b}^{2} \ell \cdot p_{a} p_{a}^{\mu} p_{a}^{\nu}\right. \\
& \left.\left.+\frac{1}{2} p_{a}^{2} p_{b}^{2}\left(\ell^{\mu} p_{a}^{\nu}+\ell^{\nu} p_{a}^{\mu}\right)\right\} \times \frac{1}{\left(\ell \cdot p_{a}+i \epsilon\right)^{2}}\right] .
\end{aligned}
$$

It will be understood that in this integral the integration over $\vec{\ell}_{\perp}$ is restricted to the region $L^{-1} \gg\left|\vec{\ell}_{\perp}\right| \gg \omega$. Since for fixed $\vec{\ell}_{\perp}$, the integration over $\ell^{0}$ and $\ell^{3}$ are finite, we do not need to impose separate cut-off on the $\ell^{0}$ and $\ell^{3}$ integrals. All the terms in (3.34) can be expressed in terms of the basic integral

$$
\int \frac{d^{4} \ell}{(2 \pi)^{4}} \frac{1}{\ell \cdot p_{b}-i \epsilon} G_{r}(\ell) \frac{1}{\left(\ell \cdot p_{a}+i \epsilon\right)^{2}} \ell_{\alpha}=-\frac{\partial}{\partial p_{a}^{\alpha}} J_{a b}
$$

where

$$
J_{a b}=\int \frac{d^{4} \ell}{(2 \pi)^{4}} \frac{1}{\ell \cdot p_{b}-i \epsilon} G_{r}(\ell) \frac{1}{\ell \cdot p_{a}+i \epsilon} .
$$


It has been shown in appendix B that $J_{a b}$ vanishes when $a$ represents an incoming particle and $b$ represents an outgoing particle or vice versa. On the other hand when $a$ and $b$ are both ingoing particles or both outgoing particles, we have, from (B.6),

$$
J_{a b}=\frac{1}{4 \pi} \ln \left\{L\left(\omega+i \epsilon \eta_{a}\right)\right\} \frac{1}{\sqrt{\left(p_{a} \cdot p_{b}\right)^{2}-p_{a}^{2} p_{b}^{2}}},
$$

where $\eta_{a}$ is a number that takes value 1 for outgoing particles $(1 \leq a \leq n)$ and -1 for incoming particles $(n+1 \leq a \leq m+n) .{ }^{5}$ Using (3.37) we can express (3.35) as

$$
\begin{aligned}
\int \frac{d^{4} \ell}{(2 \pi)^{4}} \frac{1}{\ell \cdot p_{b}-i \epsilon} G_{r}(\ell) \frac{1}{\left(\ell \cdot p_{a}+i \epsilon\right)^{2}} \ell_{\alpha} & =-\frac{1}{4 \pi} \ln \left\{L\left(\omega+i \epsilon \eta_{a}\right)\right\} \frac{\partial}{\partial p_{a}^{\alpha}} \frac{1}{\sqrt{\left(p_{a} \cdot p_{b}\right)^{2}-p_{a}^{2} p_{b}^{2}}} \\
& =-\frac{1}{4 \pi} \ln \left\{L\left(\omega+i \epsilon \eta_{a}\right)\right\} \frac{p_{b}^{2} p_{a \alpha}-p_{a} \cdot p_{b} p_{b \alpha}}{\left\{\left(p_{a} \cdot p_{b}\right)^{2}-p_{a}^{2} p_{b}^{2}\right\}^{3 / 2}} .
\end{aligned}
$$

We now use this to evaluate the right hand side of (3.34). We can also replace $e^{i k . r_{a}}$ by 1 since the difference is higher order in the small $\omega$ limit. This gives

$$
\begin{aligned}
\Delta \widehat{T}^{X \mu \nu}(k)= & 2 G \sum_{a=1}^{m+n} \sum_{\substack{b \neq a \\
\eta_{a} \eta_{b}=1}} \frac{\ln \left\{L\left(\omega+i \epsilon \eta_{a}\right)\right\}}{\left\{\left(p_{a} \cdot p_{b}\right)^{2}-p_{a}^{2} p_{b}^{2}\right\}^{3 / 2}}\left[\frac{k \cdot p_{b}}{k \cdot p_{a}} p_{a}^{\mu} p_{a}^{\nu} p_{a} \cdot p_{b}\left\{\frac{3}{2} p_{a}^{2} p_{b}^{2}-\left(p_{a} \cdot p_{b}\right)^{2}\right\}\right. \\
& \left.+\frac{1}{2} p_{a}^{\mu} p_{a}^{\nu} p_{a}^{2}\left(p_{b}^{2}\right)^{2}-\left\{p_{a}^{\mu} p_{b}^{\nu}+p_{a}^{\nu} p_{b}^{\mu}\right\} p_{a} \cdot p_{b}\left\{\frac{3}{2} p_{a}^{2} p_{b}^{2}-\left(p_{a} \cdot p_{b}\right)^{2}\right\}\right] .
\end{aligned}
$$

The constraint $\eta_{a} \eta_{b}=1$ means that the sum over $b$ runs over incoming particles if $a$ represents an incoming particle and runs over outgoing particles if $a$ represents an outgoing particle.

\subsection{Subleading contribution from the gravitational stress tensor}

Let us now turn to the computation of $T^{h \mu \nu}$ defined via (3.6). A detailed calculation shows that to quadratic order in $h_{\mu \nu}$, it has the form:

$$
\begin{aligned}
8 \pi G T^{h \mu \nu}= & -2\left[\frac{1}{2} \partial^{\mu} h_{\alpha \beta} \partial^{\nu} h^{\alpha \beta}+h^{\alpha \beta} \partial^{\mu} \partial^{\nu} h_{\alpha \beta}-h^{\alpha \beta} \partial^{\nu} \partial_{\beta} h_{\alpha}^{\mu}-h^{\alpha \beta} \partial^{\mu} \partial_{\beta} h_{\alpha}^{\nu}+h^{\alpha \beta} \partial_{\alpha} \partial_{\beta} h^{\mu \nu}\right. \\
& \left.+\partial^{\beta} h^{\nu \alpha} \partial_{\beta} h_{\alpha}^{\mu}-\partial^{\beta} h^{\alpha \nu} \partial_{\alpha} h_{\beta}^{\mu}\right]+h^{\mu \nu} \partial_{\rho} \partial^{\rho} h-2 h^{\mu}{ }_{\rho} \partial^{\sigma} \partial_{\sigma} h^{\nu \rho}-2 h^{\nu} \partial^{\sigma} \partial_{\sigma} h^{\mu \rho} \\
& +\eta^{\mu \nu}\left[\frac{3}{2} \partial^{\rho} h_{\alpha \beta} \partial_{\rho} h^{\alpha \beta}+2 h^{\alpha \beta} \partial^{\rho} \partial_{\rho} h_{\alpha \beta}-\partial^{\beta} h^{\alpha \rho} \partial_{\alpha} h_{\beta \rho}\right]+h\left[\partial^{\rho} \partial_{\rho} h^{\mu \nu}-\frac{1}{2} \partial^{\rho} \partial_{\rho} h \eta^{\mu \nu}\right],
\end{aligned}
$$

where we have used de Donder gauge condition to simplify the expression. To the order that we shall be working, this is allowed due to the observation made below (3.21). This expression differs from some of the more standard expressions given e.g. in [55], since

\footnotetext{
${ }^{5}$ This is opposite to the convention used in [18].
} 


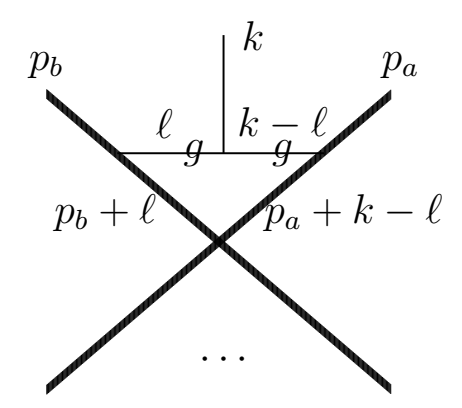

Figure 3. A pictorial representation of (3.41). The internal thin lines marked by $g$ represent the retarded graviton propagators $G_{r}(\ell)$ and $G_{r}(k-\ell)$ respectively. The vertex where the three thin lines meet is proportional to $F^{\mu \nu, \alpha \beta, \rho \sigma}$, encoding the contribution to the stress tensor due to the gravitational field, and the vertices where the internal gravitons meet the thick lines correspond to the leading contribution to the trace reversed stress tensors of these particles, proportional to $p_{a}^{\mu} p_{a}^{\nu}-p_{a}^{2} \eta^{\mu \nu} / 2$ and $p_{b}^{\mu} p_{b}^{\nu}-p_{b}^{2} \eta^{\mu \nu} / 2$ respectively.

we have defined $8 \pi G T^{h \mu \nu}$ as the collection of the quadratic terms in the expansion of $-\sqrt{-\operatorname{det} g}\left(R^{\mu \nu}-g^{\mu \nu} R / 2\right)$. As already mentioned, all indices in (3.40) are raised and lowered using the flat metric $\eta$.

We shall manipulate (3.40) by expressing $h_{\alpha \beta}$ in the momentum space as given in (3.19). This gives a general expression of the form:

$$
\begin{aligned}
\widehat{T}^{h \mu \nu}(k)= & -8 \pi G \sum_{a, b} e^{-i k \cdot r_{a}} \int \frac{d^{4} \ell}{(2 \pi)^{4}} e^{i \ell \cdot\left(r_{a}-r_{b}\right)} G_{r}(k-\ell) G_{r}(\ell) \frac{1}{p_{b} \cdot \ell-i \epsilon} \frac{1}{p_{a} \cdot(k-\ell)-i \epsilon} \\
& \times\left\{p_{b \alpha} p_{b \beta}-\frac{1}{2} p_{b}^{2} \eta_{\alpha \beta}\right\} \mathcal{F}^{\mu \nu, \alpha \beta, \rho \sigma}(k, \ell)\left\{p_{a \rho} p_{a \sigma}-\frac{1}{2} p_{a}^{2} \eta_{\rho \sigma}\right\}
\end{aligned}
$$

where,

$$
\begin{aligned}
& \mathcal{F}^{\mu \nu, \alpha \beta, \rho \sigma}(k, \ell) \\
= & 2\left[\frac{1}{2} \ell^{\mu}(k-\ell)^{\nu} \eta^{\rho \alpha} \eta^{\sigma \beta}+(k-\ell)^{\mu}(k-\ell)^{\nu} \eta^{\rho \alpha} \eta^{\sigma \beta}-(k-\ell)^{\nu}(k-\ell)^{\beta} \eta^{\rho \alpha} \eta^{\sigma \mu}\right. \\
& -(k-\ell)^{\mu}(k-\ell)^{\beta} \eta^{\rho \alpha} \eta^{\sigma \nu}+(k-\ell)^{\alpha}(k-\ell)^{\beta} \eta^{\rho \mu} \eta^{\sigma \nu}+(k-\ell) \cdot \ell \eta^{\beta \nu} \eta^{\alpha \rho} \eta^{\sigma \mu} \\
& \left.-\ell^{\rho}(k-\ell)^{\alpha} \eta^{\beta \nu} \eta^{\sigma \mu}-\frac{1}{2}(k-\ell)^{2} \eta^{\alpha \mu} \eta^{\beta \nu} \eta^{\rho \sigma}+\eta^{\alpha \mu} \eta^{\beta \rho} \eta^{\nu \sigma}(k-\ell)^{2}+\eta^{\alpha \nu} \eta^{\beta \rho} \eta^{\mu \sigma}(k-\ell)^{2}\right] \\
- & \eta^{\mu \nu}\left[\frac{3}{2}(k-\ell) \cdot \ell \eta^{\rho \alpha} \eta^{\sigma \beta}+2(k-\ell)^{2} \eta^{\rho \alpha} \eta^{\sigma \beta}-\ell^{\sigma}(k-\ell)^{\alpha} \eta^{\rho \beta}\right] \\
- & \eta^{\alpha \beta}(k-\ell)^{2} \eta^{\rho \mu} \eta^{\sigma \nu}+\frac{1}{2} \eta^{\alpha \beta}(k-\ell)^{2} \eta^{\rho \sigma} \eta^{\mu \nu} .
\end{aligned}
$$

In the $\ell^{\mu} \rightarrow 0$ limit the integrand diverges as $\left|\ell^{\mu}\right|^{-4}$ and therefore the integral has logarithmic infrared divergence. As discussed below (2.4), the lower cut-off on the $\ell^{\mu}$ integral in this case is provided by $R^{-1}$ where $R$ is the distance to the detector (measured in flat metric). Formally, this can be achieved by adding to $k^{0}=\omega$ a small imaginary part proportional to $R^{-1}$. A diagrammatic representation of (3.41) has been shown in figure 3 . 
Now in (3.41) the $G_{r}(\ell) G_{r}(k-\ell)$ factor takes the form:

$$
G_{r}(\ell) G_{r}(k-\ell)=\frac{1}{\left(\ell^{0}+i \epsilon\right)^{2}-\vec{\ell}^{2}} \frac{1}{\left(k^{0}-\ell^{0}+i \epsilon\right)^{2}-(\vec{k}-\vec{\ell})^{2}} .
$$

As a result the poles of the two denominators in the $\ell^{0}$ plane are on the opposite sides of the integration contour lying along the real axis. We shall express this as:

$$
G_{r}(\ell)^{*} G_{r}(k-\ell)-2 i \pi \delta\left(\ell^{2}\right)\left\{H\left(\ell^{0}\right)-H\left(-\ell^{0}\right)\right\} G_{r}(k-\ell),
$$

where $H$ is the Heaviside step function. In this case in the first term the poles in both factors are in the upper half $\ell^{0}$ plane. This allows us to deform the $\ell^{0}$ contour away from these poles till we hit the zeros of the other denominators. In particular, following the argument given in the paragraph containing (3.33), one can argue that for $\left|\ell_{\perp}\right|>\omega$, we can deform the contours such that it maintains a distance of order $\ell_{\perp}$ from all the poles. We shall show in appendix $\mathrm{C}$ that the contribution from the terms proportional to $\delta\left(\ell^{2}\right)$ in (3.44) represents the contribution to $\widehat{T}^{\mu \nu}$ from the gravitational radiation (real gravitons) emitted during the scattering. Since this contribution has already been included by including the radiation contribution in the sum over $a$, we shall not discuss them any further in this section.

We shall now analyze possible logarithmic contribution to (3.41) with $G_{r}(\ell)$ replaced by $G_{r}(\ell)^{*}$. These can arise from three regions: $R^{-1} \ll\left|k^{\mu}-\ell^{\mu}\right| \ll \omega, R^{-1} \ll\left|\ell^{\mu}\right| \ll \omega$ and $L^{-1} \gg\left|\ell^{\mu}\right| \gg \omega$. Since each term in (3.42) has at least one power of $(k-\ell)$, one finds by simple power counting that there is no logarithmic contribution from the region $R^{-1} \ll\left|k^{\mu}-\ell^{\mu}\right| \ll \omega$. For $R^{-1} \ll\left|\ell^{\mu}\right| \ll \omega$ the integrand has four powers of $\ell$ in the denominator and could give logarithmic contribution. In this region we can replace the integrand by its leading term in the $\ell \rightarrow 0$ limit. In particular $\mathcal{F}_{\alpha \beta ; \rho \sigma}^{\mu \nu}(k, \ell)$ may be approximated as

$$
\mathcal{F}_{\alpha \beta ; \rho \sigma}^{\mu \nu}(k, \ell) \simeq 2 k^{\mu} k^{\nu} \eta_{\alpha \rho} \eta_{\beta \sigma}-2 k^{\nu} k_{\beta} \eta_{\rho \alpha} \delta_{\sigma}^{\mu}-2 k^{\mu} k_{\beta} \eta_{\rho \alpha} \delta_{\sigma}^{\nu}+2 k_{\alpha} k_{\beta} \delta_{\rho}^{\mu} \delta_{\sigma}^{\nu},
$$

where we have used $k^{\rho} k_{\rho}=0$. A further simplification is possible by noting that eventually we shall use the $\widehat{T}^{h \mu \nu}(k)$ computed from (3.41) to calculate its contribution to subleading correction to asymptotic $\widetilde{e}_{\mu \nu}$ via (3.7). Since $\widetilde{e}_{\mu \nu}$ is determined only up to a gauge transformation

$$
\widetilde{e}_{\mu \nu} \rightarrow \widetilde{e}_{\mu \nu}+k_{\mu} \xi_{\nu}+k_{\nu} \xi_{\mu}-k \cdot \xi \eta_{\mu \nu},
$$

for any vector $\xi$, addition of a similar term to $\widehat{T}_{\mu \nu}^{h}$ and hence to $\mathcal{F}_{\alpha \beta ; \rho \sigma}^{\mu \nu}(k, \ell)$ will not have any effect of $\widetilde{e}_{\mu \nu}$. Using this we can simplify (3.45) to:

$$
\mathcal{F}_{\alpha \beta ; \rho \sigma}^{\mu \nu}(k, \ell) \simeq-2 k_{\sigma} k_{\beta} \eta_{\rho \alpha} \eta^{\mu \nu}+2 k_{\alpha} k_{\beta} \delta_{\rho}^{\mu} \delta_{\sigma}^{\nu} .
$$

We can also make the approximations:

$$
\begin{aligned}
\frac{1}{p_{a} \cdot(k-\ell)-i \epsilon} & \simeq \frac{1}{p_{a} \cdot k-i \epsilon}, \quad G_{r}(\ell)^{*}=\frac{1}{\left(\ell^{0}-i \epsilon\right)^{2}-\overrightarrow{\ell^{2}}} \\
G_{r}(k-\ell) & =\frac{1}{\left(k^{0}-\ell^{0}+i \epsilon\right)^{2}-(\vec{k}-\vec{\ell})^{2}} \simeq \frac{1}{2(k \cdot \ell+i \epsilon \omega)} \simeq \frac{1}{2(k \cdot \ell+i \epsilon)} .
\end{aligned}
$$


Substituting these into (3.41), we get the logarithmic contribution from the $R^{-1} \ll\left|\ell^{\mu}\right| \ll \omega$ region, denoted by $\widehat{T}^{(1) \mu \nu}(k)$ :

$$
\begin{aligned}
\widehat{T}^{(1) \mu \nu}(k)= & 8 \pi G \sum_{a, b=1}^{m+n} \frac{1}{p_{a} \cdot k-i \epsilon} \int \frac{d^{4} \ell}{(2 \pi)^{4}} \frac{1}{k \cdot \ell+i \epsilon} \frac{1}{p_{b} \cdot \ell-i \epsilon} \frac{1}{\left(\ell^{0}-i \epsilon\right)^{2}-\overrightarrow{\ell^{2}}} \\
& \left\{p_{a} \cdot p_{b} k \cdot p_{a} k \cdot p_{b} \eta^{\mu \nu}-\frac{1}{2} p_{b}^{2}\left(k \cdot p_{a}\right)^{2} \eta^{\mu \nu}-\left(k \cdot p_{b}\right)^{2} p_{a}^{\mu} p_{a}^{\nu}\right\} .
\end{aligned}
$$

This integral, called $K_{b}^{\prime}$ in (B.10), has been evaluated in (B.11), and gives:

$$
\int \frac{d^{4} \ell}{(2 \pi)^{4}} \frac{1}{k \cdot \ell+i \epsilon} \frac{1}{p_{b} \cdot \ell-i \epsilon} \frac{1}{\left(\ell^{0}-i \epsilon\right)^{2}-\overrightarrow{\ell^{2}}}=\frac{1}{4 \pi} \delta_{\eta_{b}, 1} \ln \{(\omega+i \epsilon) R\} \frac{1}{k \cdot p_{b}} .
$$

Using this, we get:

$$
\begin{aligned}
\widehat{T}^{(1) \mu \nu}(k)= & 2 G \ln \{(\omega+i \epsilon) R\} \sum_{a=1}^{m+n} \sum_{b=1}^{n} \frac{1}{p_{a} \cdot k-i \epsilon} \frac{1}{p_{b} \cdot k-i \epsilon} \\
& \left\{p_{a} \cdot p_{b} k \cdot p_{a} k \cdot p_{b} \eta^{\mu \nu}-\frac{1}{2} p_{b}^{2}\left(k \cdot p_{a}\right)^{2} \eta^{\mu \nu}-\left(k \cdot p_{b}\right)^{2} p_{a}^{\mu} p_{a}^{\nu}\right\} .
\end{aligned}
$$

The terms proportional to $p_{a} \cdot k p_{b} . k$ and $\left(k \cdot p_{a}\right)^{2}$ inside the curly bracket cancel the denominator factor of $k \cdot p_{a}$, and the result vanishes by momentum conservation after summing over $a$. Therefore we have

$$
\widehat{T}^{(1) \mu \nu}(k)=-2 G \ln \{(\omega+i \epsilon) R\} \sum_{a=1}^{m+n} \sum_{b=1}^{n} \frac{p_{b} \cdot k}{p_{a} \cdot k-i \epsilon} p_{a}^{\mu} p_{a}^{\nu} .
$$

Next we turn to the contribution from the region $L^{-1} \gg\left|\ell^{\mu}\right| \gg \omega$. Simple power counting shows that the integrand goes as $|\ell|^{-4}$ in this region. Therefore, in order to extract the logarithmic term, we need to keep only the leading term in the integrand for large $\ell^{\mu}$. In particular, in the expression for $\mathcal{F}^{\mu \nu, \alpha \beta, \rho \sigma}(k, \ell)$, we need to keep only quadratic terms in $\ell$. Therefore we need to evaluate the integral:

$$
\mathcal{I}_{a b}^{\alpha \beta} \equiv \int \frac{d^{4} \ell}{(2 \pi)^{4}} e^{i \ell \cdot\left(r_{a}-r_{b}\right)} G_{r}(k-\ell) G_{r}(\ell)^{*} \frac{1}{p_{b} \cdot \ell-i \epsilon} \frac{1}{p_{a} \cdot(\ell-k)+i \epsilon} \ell^{\alpha} \ell^{\beta} .
$$

Using $L^{-1} \gg\left|\ell^{\mu}\right| \gg \omega$, we can further approximate (3.53) by

$$
\begin{aligned}
\mathcal{I}_{a b}^{\alpha \beta} & \simeq \int \frac{d^{4} \ell}{(2 \pi)^{4}} \frac{1}{\left\{\left(\ell^{0}-i \epsilon\right)^{2}-\vec{\ell}^{2}\right\}^{2}} \frac{1}{p_{b} \cdot \ell-i \epsilon} \frac{1}{p_{a} \cdot \ell+i \epsilon} \ell^{\alpha} \ell^{\beta} \\
& =\frac{1}{2} \int \frac{d^{4} \ell}{(2 \pi)^{4}} \frac{\partial}{\partial \ell_{\alpha}}\left\{\frac{1}{\left(\ell^{0}-i \epsilon\right)^{2}-\overrightarrow{\ell^{2}}}\right\} \frac{1}{p_{b} \cdot \ell-i \epsilon} \frac{1}{p_{a} \cdot \ell+i \epsilon} \ell^{\beta} \\
& =-\frac{1}{2} \int \frac{d^{4} \ell}{(2 \pi)^{4}} \frac{1}{\left(\ell^{0}-i \epsilon\right)^{2}-\overrightarrow{\ell^{2}}} \frac{1}{p_{b} \cdot \ell-i \epsilon} \frac{1}{p_{a} \cdot \ell+i \epsilon}\left\{\eta^{\alpha \beta}-\frac{p_{a}^{\alpha} \ell^{\beta}}{p_{a} \cdot \ell+i \epsilon}-\frac{p_{b}^{\alpha} \ell^{\beta}}{p_{b} \cdot \ell-i \epsilon}\right\} \\
& =-\frac{1}{2}\left\{\eta^{\alpha \beta}+p_{a}^{\alpha} \frac{\partial}{p_{a \beta}}+p_{b}^{\alpha} \frac{\partial}{\partial p_{b \beta}}\right\} \int \frac{d^{4} \ell}{(2 \pi)^{4}} \frac{1}{\left(\ell^{0}-i \epsilon\right)^{2}-\overrightarrow{\ell^{2}}} \frac{1}{p_{a} \cdot \ell+i \epsilon} \frac{1}{p_{b} \cdot \ell-i \epsilon} \cdot
\end{aligned}
$$


In the third step we have carried out an integration by parts. ${ }^{6}$ We can now evaluate the integral using the result for $J_{a b}$ given in (B.1). The result vanishes when $a$ represents an ingoing particle and $b$ represents an outgoing particle or vice versa. When both particles are ingoing or both particles are outgoing, the result is given in (B.6). This gives

$$
\begin{aligned}
I_{a b}^{\alpha \beta} \simeq & -\frac{1}{8 \pi} \ln \left\{L\left(\omega+i \epsilon \eta_{a}\right)\right\}\left\{\eta^{\alpha \beta}+p_{a}^{\alpha} \frac{\partial}{p_{a \beta}}+p_{b}^{\alpha} \frac{\partial}{\partial p_{b \beta}}\right\} \frac{1}{\sqrt{\left(p_{a} \cdot p_{b}\right)^{2}-p_{a}^{2} p_{b}^{2}}} \\
= & -\frac{1}{8 \pi} \ln \left\{L\left(\omega+i \epsilon \eta_{a}\right)\right\} \frac{1}{\left\{\left(p_{a} \cdot p_{b}\right)^{2}-p_{a}^{2} p_{b}^{2}\right\}^{3 / 2}} \\
& \times\left[\eta^{\alpha \beta}\left\{\left(p_{a} \cdot p_{b}\right)^{2}-p_{a}^{2} p_{b}^{2}\right\}+p_{a}^{2} p_{b}^{\alpha} p_{b}^{\beta}+p_{b}^{2} p_{a}^{\alpha} p_{a}^{\beta}-p_{a} \cdot p_{b}\left(p_{a}^{\alpha} p_{b}^{\beta}+p_{a}^{\beta} p_{b}^{\alpha}\right)\right] .
\end{aligned}
$$

Note that the result diverges for $a=b$. This can be traced to the fact that if we replace the $p_{a} \cdot(\ell-k)+i \epsilon$ factor in the denominator of (3.53) by $p_{a} \cdot \ell+i \epsilon$ from the beginning, then for $b=a$ the contour is pinched by the poles from both sides with separation of order $\epsilon$, and we shall get a divergence in the $\epsilon \rightarrow 0$ limit. This shows that for $a=b$ we have to be more careful in evaluating the integral. We proceed by working with (3.53) without making any approximation at the beginning. If we work in the rest frame of $p_{a}$, then we can evaluate the $\ell^{0}$ integral by closing the contour in the lower half plane, picking the residue at $\ell^{0}=0$ for outgoing $p_{a}=p_{b}$ and at $\ell^{0}=k^{0}$ for incoming $p_{a}=p_{b}$. Let us for definiteness consider the case where the particle is outgoing, so that we pick up the residue from the pole at $\ell^{0}=0$. This reduces the integral to

$$
I_{a a}^{\alpha \beta}=-2 \pi i \frac{1}{p_{a}^{0}} \frac{1}{p_{a} \cdot k-i \epsilon} \int \frac{d^{3} \ell}{(2 \pi)^{4}} \frac{1}{\vec{\ell}^{2}} \frac{1}{\overrightarrow{\ell^{2}}-2 \vec{k} \cdot \vec{\ell}-i \epsilon} \ell^{\alpha} \ell^{\beta} .
$$

Since this is potentially linearly divergent from the region of large $|\vec{\ell}|$, we expand the integrand in power series expansion in inverse powers of $\ell$, keeping up to the first subleading term:

$$
I_{a a}^{\alpha \beta} \simeq-2 \pi i \frac{1}{p_{a}^{0}} \frac{1}{p_{a} \cdot k-i \epsilon} \int \frac{d^{3} \ell}{(2 \pi)^{4}} \frac{1}{\overrightarrow{\ell^{2}}}\left[\frac{1}{\overrightarrow{\ell^{2}}}+\frac{2 \vec{k} \cdot \vec{\ell}}{\left(\overrightarrow{\ell^{2}}\right)^{2}}\right] \ell^{\alpha} \ell^{\beta} .
$$

The leading linearly divergent term, where we pick the $\ell^{\alpha} \ell^{\beta}$ term from the numerator, represents the usual infinite self energy of a classical point particle, and is regulated by the intrinsic size of the particle. In any case, this does not lead to any logarithmic terms. The potentially logarithmically divergent subleading contribution actually vanishes by $\vec{\ell} \rightarrow-\vec{\ell}$ symmetry since it has to be evaluated at $\ell^{0}=0$. Therefore we conclude that $I_{a a}^{\alpha \beta}$ does not have any logarithmic correction. A similar analysis can be carried out for the incoming particles, leading to the same conclusion.

\footnotetext{
${ }^{6}$ This can be justified as follows. First, following arguments similar to the one given below (3.32), we can consider the integration region to be $\omega \ll\left|\ell_{\perp}\right| \ll L^{-1}$, without any restriction on $\ell^{0}$ and $\ell^{3}$. Integration by parts will then give boundary contributions from $\left|\ell_{\perp}\right|=\omega$ and $\left|\ell_{\perp}\right|=L^{-1}$. These involve angular integration and do not generate any logarithmic terms.
} 
Substituting (3.55) into (3.41) for $a \neq b$, we get the logarithmic contribution to $\widehat{T}^{h \mu \nu}(k)$ from the region $\omega \ll\left|\ell^{\mu}\right| \ll L^{-1}$, which we shall denote by $\widehat{T}^{(2) \mu \nu}(k)$ :

$$
\begin{aligned}
\widehat{T}^{(2) \mu \nu}(k)= & G \sum_{a=1}^{m+n} \ln \left\{L\left(\omega+i \epsilon \eta_{a}\right)\right\} \sum_{\substack{b=1 \\
b \neq a, \eta_{a} \eta_{b}=1}}^{m+n} \frac{1}{\left\{\left(p_{a} \cdot p_{b}\right)^{2}-p_{a}^{2} p_{b}^{2}\right\}^{3 / 2}} \\
& \times\left[-p_{b}^{\mu} p_{b}^{\nu}\left(p_{a}^{2}\right)^{2}\left(p_{b}^{2}\right)+\left\{p_{a}^{\mu} p_{b}^{\nu}+p_{a}^{\nu} p_{b}^{\mu}\right\} p_{a} \cdot p_{b}\left\{\frac{3}{2} p_{a}^{2} p_{b}^{2}-\left(p_{a} \cdot p_{b}\right)^{2}\right\}\right] .
\end{aligned}
$$

\subsection{Gravitational wave-form at early and late time}

Adding (3.17), (3.39), (3.52) and (3.58) we get the net logarithmic contribution to $\widehat{T}^{\mu \nu}(k)$ to the subleading order in the small $\omega$ expansion: ${ }^{7}$

$$
\begin{aligned}
& \widehat{T}^{\mu \nu}(k) \\
& =\sum_{a=1}^{n} p_{a}^{\mu} p_{a}^{\nu} \frac{1}{i\left(k \cdot p_{a}-i \epsilon\right)}-\sum_{a=1}^{m} p_{a}^{\prime \mu} p_{a}^{\prime \nu} \frac{1}{i\left(k \cdot p_{a}^{\prime}+i \epsilon\right)} \\
& +2 G \ln \{L(\omega+i \epsilon)\} \sum_{a=1}^{n} \sum_{\substack{b=1 \\
b \neq a}}^{n} \frac{p_{a} \cdot p_{b}}{\left\{\left(p_{a} \cdot p_{b}\right)^{2}-p_{a}^{2} p_{b}^{2}\right\}^{3 / 2}}\left\{\frac{3}{2} p_{a}^{2} p_{b}^{2}-\left(p_{a} \cdot p_{b}\right)^{2}\right\} \frac{k_{\rho} p_{a}^{\mu}}{k \cdot p_{a}}\left(p_{b}^{\rho} p_{a}^{\nu}-p_{b}^{\nu} p_{a}^{\rho}\right) \\
& +2 G \ln \{L(\omega-i \epsilon)\} \sum_{a=1}^{m} \sum_{\substack{b=1 \\
b \neq a}}^{m} \frac{p_{a}^{\prime} \cdot p_{b}^{\prime}}{\left\{\left(p_{a}^{\prime} \cdot p_{b}^{\prime}\right)^{2}-p_{a}^{\prime 2} p_{b}^{\prime 2}\right\}^{3 / 2}}\left\{\frac{3}{2} p_{a}^{\prime 2} p_{b}^{\prime 2}-\left(p_{a}^{\prime} \cdot p_{b}^{\prime}\right)^{2}\right\} \frac{k_{\rho} p_{a}^{\prime \mu}}{k \cdot p_{a}^{\prime}}\left(p_{b}^{\prime \rho} p_{a}^{\prime \nu}-p_{b}^{\prime \nu} p_{a}^{\prime \rho}\right) \\
& -2 G \ln \{(\omega+i \epsilon) R\} \sum_{b=1}^{n} p_{b} \cdot k\left[\sum_{a=1}^{n} \frac{1}{p_{a} \cdot k-i \epsilon} p_{a}^{\mu} p_{a}^{\nu}-\sum_{a=1}^{m} \frac{1}{p_{a}^{\prime} \cdot k-i \epsilon} p_{a}^{\prime \mu} p_{a}^{\prime \nu}\right] .
\end{aligned}
$$

In (3.59) we can replace $k$ by $\omega n$ with $n=(1, \hat{n})$. Comparing the second and last line of (3.59) we see that the term proportional to $\ln R$ exponentiates to a multiplicative factor of

$$
\exp \left[-2 i \omega G \ln R \sum_{b=1}^{n} p_{b} . n\right] \text {. }
$$

Using (3.7), (2.5) and (2.6) we get the late and early time behaviour of the gravitational wave-form:

$$
\begin{aligned}
\text { Late time : } & e^{\mu \nu}(t, R, \hat{n})=\frac{2 G}{R}\left[-\sum_{a=1}^{n} p_{a}^{\mu} p_{a}^{\nu} \frac{1}{n \cdot p_{a}}+\sum_{a=1}^{m} p_{a}^{\prime \mu} p_{a}^{\prime \nu} \frac{1}{n \cdot p_{a}^{\prime}}\right] \\
& -\frac{4 G^{2}}{R u}\left[\sum_{\substack { a=1 \\
\begin{subarray}{c}{b=1 \\
b \neq a{ a = 1 \\
\begin{subarray} { c } { b = 1 \\
b \neq a } }\end{subarray}}^{n} \frac{p_{a} \cdot p_{b}}{\left\{\left(p_{a} \cdot p_{b}\right)^{2}-p_{a}^{2} p_{b}^{2}\right\}^{3 / 2}}\left\{\frac{3}{2} p_{a}^{2} p_{b}^{2}-\left(p_{a} \cdot p_{b}\right)^{2}\right\} \frac{n_{\rho} p_{a}^{\mu}}{n \cdot p_{a}}\left(p_{b}^{\rho} p_{a}^{\nu}-p_{b}^{\nu} p_{a}^{\rho}\right)\right. \\
& \left.-\sum_{b=1}^{n} p_{b} \cdot n\left\{\sum_{a=1}^{n} \frac{1}{p_{a} \cdot n} p_{a}^{\mu} p_{a}^{\nu}-\sum_{a=1}^{m} \frac{1}{p_{a}^{\prime} \cdot n} p_{a}^{\prime \mu} p_{a}^{\prime \nu}\right\}\right], \quad \text { as } u \rightarrow \infty,
\end{aligned}
$$

\footnotetext{
${ }^{7}$ The quantum computation of [18] gave rise to additional terms in the soft factor, but they do not seem to play any role in the classical gravitational wave-form found here.
} 
Early time : $e^{\mu \nu}(t, R, \hat{n})=\frac{4 G^{2}}{R u}\left[\sum_{\substack{a=1 \\ \sum_{b=1}}}^{m} \frac{p_{a}^{\prime} \cdot p_{b}^{\prime}}{\left\{\left(p_{a}^{\prime} \cdot p_{b}^{\prime}\right)^{2}-p_{a}^{\prime 2} p_{b}^{\prime 2}\right\}^{3 / 2}}\left\{\frac{3}{2} p_{a}^{\prime 2} p_{b}^{\prime 2}-\left(p_{a}^{\prime} \cdot p_{b}^{\prime}\right)^{2}\right\}\right.$

$$
\left.\times \frac{n_{\rho} p_{a}^{\prime \mu}}{n \cdot p_{a}^{\prime}}\left(p_{b}^{\prime \rho} p_{a}^{\prime \nu}-p_{b}^{\prime \nu} p_{a}^{\prime \rho}\right)\right], \quad \text { as } u \rightarrow-\infty
$$

where, from (A.12) and (3.7), (3.60),

$$
u=t-R+2 G \ln R \sum_{b=1}^{n} p_{b} . n .
$$

In (3.61) we have adjusted the overall additive constant in the expression for $e^{\mu \nu}$ such that it vanishes in the far past.

\section{Generalizations}

In this section we shall derive the classical soft photon theorem. We shall also generalize the soft graviton theorem to include the effect of electromagnetic interactions among the incoming and the outgoing particles. In order to simplify our formulæ we shall drop the regulator factors of $e^{i \ell .\left(r_{a}-r_{b}\right)}, e^{i k \cdot r_{a}}$ etc., with the understanding that momentum integrals have an upper cut-off $L^{-1}$ and a lower cut-off $R^{-1}$.

\subsection{Soft photon theorem with electromagnetic interactions}

As in section 3, we consider a scattering event in asymptotically flat space-time in which $m$ particles carrying masses $\left\{m_{a}^{\prime} ; 1 \leq a \leq m\right\}$, four velocities $\left\{v_{a}^{\prime}\right\}$, four momenta $\left\{p_{a}^{\prime}=\right.$ $\left.m_{a}^{\prime} v_{a}^{\prime}\right\}$ and charges $\left\{q_{a}^{\prime}\right\}$ come close, undergo interactions, and disperse as $n$ particles carrying masses $\left\{m_{a} ; 1 \leq a \leq n\right\}$, four velocities $\left\{v_{a}\right\}$, four momenta $\left\{p_{a}\right\}$ and charges $\left\{q_{a}\right\}$. Our goal will be to compute the early and late time electromagnetic wave-form emitted during this scattering event. In this section we shall proceed by ignoring the gravitational interaction between the particles, but this will be included in section 4.2. Since the analysis proceeds as in section 3, we shall be brief, pointing out only the main differences. In particular, as in section 3, we can treat the incoming particles as outgoing particles with four velocities $\left\{-v_{a}^{\prime}\right\}$, four momenta $\left\{-p_{a}^{\prime}\right\}$ and charges $\left\{-q_{a}^{\prime}\right\}$. This allows us to drop the sum over incoming particles by extending the sum over $a$ from 1 to $m+n$.

In the Lorentz gauge $\eta^{\alpha \beta} \partial_{\alpha} a_{\beta}=0$, the equations replacing (3.13) are:

$$
\begin{aligned}
j^{\mu}(x) & =\sum_{a} q_{a} \int d \sigma \delta^{(4)}\left(x-X_{a}(\sigma)\right) \frac{d X_{a}^{\mu}}{d \sigma}, \quad \square a_{\mu}=-j_{\mu}, \\
m_{a} \frac{d^{2} X_{a}^{\mu}}{d \sigma^{2}} & =q_{a} F_{\nu}^{\mu}\left(X_{a}(\sigma)\right) \frac{d X_{a}^{\nu}}{d \sigma} .
\end{aligned}
$$

We introduce the Fourier transforms via:

$$
a_{\mu}(x)=\int \frac{d^{4} k}{(2 \pi)^{4}} e^{i k \cdot x} \widehat{a}_{\mu}(k), \quad j_{\mu}(x)=\int \frac{d^{4} k}{(2 \pi)^{4}} e^{i k \cdot x} \widehat{j}_{\mu}(k)
$$


This gives:

$$
\widehat{j}^{\mu}(k)=\int d^{4} x e^{-i k \cdot x} j^{\mu}(x)=\sum_{a} q_{a} \int d \sigma e^{-i k \cdot X(\sigma)} \frac{d X_{a}^{\mu}}{d \sigma} .
$$

The generalization of (3.7) for the asymptotic electromagnetic field is:

$$
\tilde{a}^{\mu}(\omega, R, \hat{n})=\frac{1}{4 \pi R} e^{i \omega R} \widehat{j}^{\mu}(k)+\mathcal{O}\left(R^{-2}\right) .
$$

We proceed to find iterative solutions to (4.1) in a power series expansion in the charges, beginning with the leading order solution for $X^{\mu}$ given in (3.11). Substituting this into (4.3) we find the leading order expression for $\widehat{j}^{\mu}(k)$ :

$$
\widehat{j}^{\mu}(k)=\sum_{a=1}^{m+n} q_{a} p_{a}^{\mu} \frac{1}{i\left(k \cdot p_{a}-i \epsilon\right)} .
$$

This is the leading soft factor. Using this we can get the analogs of (3.19) and (3.20):

$$
\begin{aligned}
& a_{\mu}^{(b)}(x)=-\int \frac{d^{4} \ell}{(2 \pi)^{4}} e^{i \ell \cdot x} G_{r}(\ell) q_{b} p_{b \mu} \frac{1}{i\left(\ell \cdot p_{b}-i \epsilon\right)} \\
& F_{\nu \rho}^{(b)}(x)=\partial_{\nu} a_{\rho}^{(b)}-\partial_{\rho} a_{\nu}^{(b)}=-\int \frac{d^{4} \ell}{(2 \pi)^{4}} e^{i \ell \cdot x} G_{r}(\ell) \frac{q_{b}}{\left(\ell \cdot p_{b}-i \epsilon\right)}\left(\ell_{\nu} p_{b \rho}-\ell_{\rho} p_{b \nu}\right)
\end{aligned}
$$

where $a_{\mu}^{(b)}$ and $F_{\mu \nu}^{(b)}$ denote the gauge field and field strength produced by the $b$-th particle. The analogs of (3.26), (3.27) take the form:

$$
\frac{d Y_{a}^{\mu}(\sigma)}{d \sigma}=-\frac{q_{a}}{m_{a}} \int_{\sigma}^{\infty} d \sigma^{\prime} F_{\nu}^{\mu}\left(v_{a} \sigma^{\prime}+r_{a}\right) v_{a}^{\nu}
$$

and

$$
Y_{a}^{\mu}(\sigma)=-\frac{q_{a}}{m_{a}} \int_{0}^{\sigma} d \sigma^{\prime} \int_{\sigma^{\prime}}^{\infty} d \sigma^{\prime \prime} F_{\nu}^{\mu}\left(v_{a} \sigma^{\prime \prime}+r_{a}\right) v_{a}^{\nu}
$$

Using these results we can proceed as in section 3.4 to compute the next order correction $\Delta \widehat{j}^{\mu}(k)$ to $\widehat{j}^{\mu}(k)$. Since the analysis is identical to those in section 3.4 we only quote the analog of (3.32):

$$
\begin{aligned}
\Delta \widehat{j}^{\mu}(k)= & \sum_{a=1}^{m+n} \sum_{\substack{b=1 \\
b \neq a}}^{m+n} q_{a}^{2} q_{b} \int \frac{d^{4} \ell}{(2 \pi)^{4}} \frac{1}{\ell \cdot p_{b}-i \epsilon} G_{r}(\ell) \\
\times & {\left[k_{\rho} \frac{1}{(k-\ell) \cdot p_{a}-i \epsilon} \frac{1}{k \cdot p_{a}-i \epsilon} \frac{1}{\ell \cdot p_{a}+i \epsilon}\left(\ell_{\nu} p_{b \rho}-\ell_{\rho} p_{b \nu}\right) p_{a}^{\nu} p_{a}^{\mu}\right.} \\
& \left.\quad-\frac{1}{(k-\ell) \cdot p_{a}-i \epsilon} \frac{1}{\ell \cdot p_{a}+i \epsilon}\left(\ell_{\nu} p_{b}^{\mu}-\ell^{\mu} p_{b \nu}\right) p_{a}^{\nu}\right] .
\end{aligned}
$$


This can be evaluated exactly as in section 3.4, leading to the final result analogous to $(3.39)$ :

$$
\begin{aligned}
\Delta \widehat{j}^{\mu}(k)= & \frac{1}{4 \pi} \ln (\omega+i \epsilon) \sum_{a=1}^{n} \sum_{\substack{b=1 \\
b \neq a}}^{n} q_{a}^{2} q_{b} p_{a}^{2} p_{b}^{2} \frac{1}{\left\{\left(p_{a} \cdot p_{b}\right)^{2}-p_{a}^{2} p_{b}^{2}\right\}^{3 / 2}} \frac{k^{\rho}}{k \cdot p_{a}}\left\{p_{b \rho} p_{a}^{\mu}-p_{a \rho} p_{b}^{\mu}\right\} \\
& +\frac{1}{4 \pi} \ln (\omega-i \epsilon) \sum_{\substack{a=1 \\
m}}^{m} \sum_{\substack{b=1 \\
b \neq a}}^{m} q_{a}^{\prime 2} q_{b}^{\prime} p_{a}^{\prime 2} p_{b}^{\prime 2} \frac{1}{\left\{\left(p_{a}^{\prime} \cdot p_{b}^{\prime}\right)^{2}-p_{a}^{\prime 2} p_{b}^{\prime 2}\right\}^{3 / 2}} \frac{k^{\rho}}{k \cdot p_{a}^{\prime}}\left\{p_{b \rho}^{\prime} p_{a}^{\prime \mu}-p_{a \rho}^{\prime} p_{b}^{\prime \mu}\right\}
\end{aligned}
$$

\subsection{Gravitational contribution to the soft photon theorem}

We shall now study the effect of gravitational interaction on the soft photon theorem. This modifies the last two equations in (4.1) as follows. First of all the equation for $a_{\mu}$ get modified to:

$$
\partial_{\nu}\left(\sqrt{-\operatorname{det} g} g^{\nu \rho} g^{\mu \sigma} F_{\rho \sigma}\right)=-j^{\mu} .
$$

Using Lorentz gauge condition $\eta^{\rho \sigma} \partial_{\rho} a_{\sigma}=0$, this may be written as

$$
\eta^{\mu \nu} \eta^{\rho \sigma} \partial_{\rho} \partial_{\sigma} a_{\nu}=-j^{\mu}-j_{h}^{\mu}
$$

where

$$
j_{h}^{\mu} \equiv \partial_{\nu}\left\{\eta^{\alpha \beta} h_{\alpha \beta} \eta^{\nu \rho} \eta^{\mu \sigma} F_{\rho \sigma}-2\left(h^{\nu \rho} \eta^{\mu \sigma}+\eta^{\nu \rho} h^{\mu \sigma}\right) F_{\rho \sigma}\right\}+\text { higher order terms } .
$$

The equation for $X^{\mu}$ is modified to:

$$
m_{a} \frac{d^{2} X_{a}^{\mu}}{d \sigma^{2}}=q_{a} F_{\nu}^{\mu}\left(X_{a}(\sigma)\right) \frac{d X_{a}^{\nu}}{d \sigma}-m_{a} \Gamma_{\nu \rho}^{\mu}\left(X_{a}(\sigma)\right) \frac{d X_{a}^{\nu}}{d \sigma} \frac{d X_{a}^{\rho}}{d \sigma} .
$$

We shall now expand the above equations in powers of $h_{\alpha \beta}$ and then raise and lower all indices by the flat metric $\eta$. We begin with the analysis of (4.15). To the order that we are working, we can study the effect of the two terms on the right hand side of (4.15) separately. The effect of the first term on $\widehat{j}^{\mu}$ has already been analyzed in section 4.1. The effect of the second term on $\widehat{T}_{\mu \nu}^{X}$ has been studied in section 3.4 , but this can be easily extended to $\widehat{j}^{\mu}$. The additional contribution to $\widehat{j}^{\mu}$ is given by:

$$
\begin{aligned}
\Delta^{(1)} \widehat{j}^{\mu}(k)= & -8 \pi G \sum_{a=1}^{m+n} \sum_{\substack{b=1 \\
b \neq a}}^{m+n} q_{a} \int \frac{d^{4} \ell}{(2 \pi)^{4}} G_{r}(\ell) \frac{1}{\ell \cdot p_{b}-i \epsilon} \\
& {\left[-\frac{\left\{2 k \cdot p_{b} \ell \cdot p_{a} p_{a} \cdot p_{b}-k \cdot \ell\left(p_{a} \cdot p_{b}\right)^{2}-\frac{1}{2}\left(2 k \cdot p_{a} \ell \cdot p_{a}-k \cdot \ell p_{a}^{2}\right) p_{b}^{2}\right\} p_{a}^{\mu}}{\left[k \cdot p_{a}-i \epsilon\right]\left[(k-\ell) \cdot p_{a}-i \epsilon\right]\left[\ell \cdot p_{a}+i \epsilon\right]}\right.} \\
& \left.+\frac{2 p_{b}^{\mu} \ell \cdot p_{a} p_{a} \cdot p_{b}-\ell^{\mu}\left(p_{a} \cdot p_{b}\right)^{2}-\frac{1}{2}\left(2 p_{a}^{\mu} \ell \cdot p_{a}-\ell^{\mu} p_{a}^{2}\right) p_{b}^{2}}{\left[(k-\ell) \cdot p_{a}-i \epsilon\right]\left[\ell \cdot p_{a}+i \epsilon\right]}\right]
\end{aligned}
$$


This integral can be evaluated as in section 3.4 and yields the result:

$$
\begin{aligned}
& \Delta^{(1)} \widehat{j}^{\mu}(k) \\
= & -G \log (\omega+i \epsilon) \sum_{a=1}^{n} \sum_{\substack{b=1 \\
b \neq a}}^{n} q_{a} \frac{k_{\rho}}{k \cdot p_{a}} \frac{p_{a} \cdot p_{b}}{\left\{\left(p_{a} \cdot p_{b}\right)^{2}-p_{a}^{2} p_{b}^{2}\right\}^{\frac{3}{2}}}\left(p_{a}^{\mu} p_{b}^{\rho}-p_{a}^{\rho} p_{b}^{\mu}\right)\left\{2\left(p_{a} \cdot p_{b}\right)^{2}-3 p_{a}^{2} p_{b}^{2}\right\} \\
& -G \log (\omega-i \epsilon) \sum_{\substack{a=1 \\
a}}^{m} \sum_{\substack{b=1 \\
b \neq a}}^{m} q_{a}^{\prime} \frac{k_{\rho}}{k \cdot p_{a}^{\prime}} \frac{p_{a}^{\prime} \cdot p_{b}^{\prime}}{\left\{\left(p_{a}^{\prime} \cdot p_{b}^{\prime}\right)^{2}-p_{a}^{\prime 2} p_{b}^{\prime 2}\right\}^{\frac{3}{2}}}\left(p_{a}^{\prime \mu} p_{b}^{\prime \rho}-p_{a}^{\prime \rho} p_{b}^{\prime \mu}\right)\left\{2\left(p_{a}^{\prime} \cdot p_{b}^{\prime}\right)^{2}-3 p_{a}^{\prime 2} p_{b}^{\prime 2}\right\} .
\end{aligned}
$$

We now turn to the evaluation of $j_{h}^{\mu}$ given in (4.14). Using the expressions for $h_{\mu \nu}$ and $F_{\mu \nu}$ given in (3.19) and (4.7), we get:

$$
\widehat{j}_{h}^{\mu}(k)=8 \pi G \sum_{a, b=1}^{m+n} q_{b} \int \frac{d^{4} \ell}{(2 \pi)^{4}} G_{r}(\ell) G_{r}(k-\ell) \frac{1}{\left[\ell \cdot p_{a}-i \epsilon\right]\left[(k-\ell) \cdot p_{b}-i \epsilon\right]} \mathcal{F}^{\mu}(k, \ell),
$$

where

$$
\begin{aligned}
\mathcal{F}^{\mu} \equiv & p_{b}^{\mu}\left\{p_{a}^{2} k \cdot(k-\ell)-2 p_{a} \cdot(k-\ell) p_{a} \cdot k\right\}+\left(k^{\mu}-\ell^{\mu}\right)\left\{2 k \cdot p_{a} p_{a} \cdot p_{b}-p_{a}^{2} k \cdot p_{b}\right\} \\
& +p_{a}^{\mu}\left\{2 p_{a} \cdot(k-\ell) k \cdot p_{b}-2 k \cdot(k-\ell) p_{a} \cdot p_{b}\right\}
\end{aligned}
$$

We shall analyze this by expressing $G_{r}(\ell) G_{r}(k-\ell)$ as in (3.44). The term proportional to $\delta\left(\ell^{2}\right)$ can be analyzed as in appendix $\mathrm{C}$, and one can show that in this case there is no contribution from this term. This gives

$$
\widehat{j}_{h}^{\mu}(k)=8 \pi G \sum_{a, b=1}^{m+n} q_{b} \int \frac{d^{4} \ell}{(2 \pi)^{4}} G_{r}(\ell)^{*} G_{r}(k-\ell) \frac{1}{\left[\ell \cdot p_{a}-i \epsilon\right]\left[(k-\ell) \cdot p_{b}-i \epsilon\right]} \mathcal{F}^{\mu}(k, \ell)
$$

This integral could give logarithmic contributions from three regions: $R^{-1} \ll\left|\ell^{\mu}\right| \ll \omega$, $R^{-1} \ll\left|k^{\mu}-\ell^{\mu}\right| \ll \omega$ and $\omega \ll\left|\ell^{\mu}\right| \ll L^{-1}$. However, since $\mathcal{F}^{\nu}$ vanishes as $k-\ell \rightarrow 0$, there is no logarithmic divergence from the $R^{-1} \ll\left|k^{\mu}-\ell^{\mu}\right| \ll \omega$ region. Furthermore, since $\mathcal{F}^{\nu}$ does not have any quadratic term in $\ell$, this rules out logarithmic contribution from the region $\omega \ll\left|\ell^{\mu}\right| \ll L^{-1}$. Therefore the only possible source of logarithmic divergence is the region $R^{-1} \ll\left|\ell^{\mu}\right| \ll \omega$. In this region,

$$
\mathcal{F}^{\nu} \simeq-2\left\{\left(k \cdot p_{a}\right)^{2} p_{b}^{\nu}-k \cdot p_{a} k \cdot p_{b} p_{a}^{\nu}\right\}
$$


We have ignored the terms proportional to $k^{\nu}$ because such terms can be removed by gauge transformation $a_{\mu} \rightarrow a_{\mu}+\partial_{\mu} \phi$ for appropriate function $\phi$. We can now evaluate the logarithmic contribution to the integral using the method described below (3.47), and the result is

$$
\widehat{j}_{h}^{\mu}(k)=-2 G \ln (\omega+i \epsilon) \sum_{a=1}^{n} k \cdot p_{a}\left[\sum_{b=1}^{n} q_{b} p_{b}^{\mu} \frac{1}{k \cdot p_{b}}-\sum_{b=1}^{m} q_{b}^{\prime} p_{b}^{\prime \mu} \frac{1}{k \cdot p_{b}^{\prime}}\right]
$$

after using charge conservation $\sum_{b=1}^{m+n} q_{b}=0$.

\subsection{Electromagnetic wave-form at early and late time}

Adding (4.5), (4.11), (4.17) and (4.22), and using $k=\omega n$ and (4.4), (2.5), (2.6) we get

$$
\begin{aligned}
4 \pi R a^{\mu}(t, R, \hat{n}) \simeq & -\sum_{a=1}^{n} q_{a} p_{a}^{\mu} \frac{1}{n \cdot p_{a}}+\sum_{a=1}^{m} q_{a}^{\prime} p_{a}^{\prime \mu} \frac{1}{n \cdot p_{a}^{\prime}} \\
& +\frac{1}{u}\left[-\frac{1}{4 \pi} \sum_{\substack{a=1 \\
\sum_{b=1}}}^{n} q_{a}^{2} q_{b} p_{a}^{2} p_{b}^{2} \frac{1}{\left\{\left(p_{a} \cdot p_{b}\right)^{2}-p_{a}^{2} p_{b}^{2}\right\}^{3 / 2}} \frac{n^{\rho}}{n \cdot p_{a}}\left\{p_{b \rho} p_{a}^{\mu}-p_{a \rho} p_{b}^{\mu}\right\}\right. \\
& +G \sum_{a=1}^{n} \sum_{\substack{b=1 \\
b \neq a}}^{n} q_{a} \frac{n_{\rho}}{n \cdot p_{a}} \frac{p_{a} \cdot p_{b}}{\left\{\left(p_{a} \cdot p_{b}\right)^{2}-p_{a}^{2} p_{b}^{2}\right\}^{\frac{3}{2}}}\left(p_{a}^{\mu} p_{b}^{\rho}-p_{a}^{\rho} p_{b}^{\mu}\right)\left\{2\left(p_{a} \cdot p_{b}\right)^{2}-3 p_{a}^{2} p_{b}^{2}\right\} \\
& \left.+2 G \sum_{a=1}^{n} n \cdot p_{a}\left\{\sum_{b=1}^{n} q_{b} p_{b}^{\mu} \frac{1}{n \cdot p_{b}}-\sum_{b=1}^{m} q_{b}^{\prime} p_{b}^{\prime \mu} \frac{1}{n \cdot p_{b}^{\prime}}\right\}\right], \quad \text { as } u \rightarrow \infty, \quad(4.23)
\end{aligned}
$$

and

$$
\begin{aligned}
& 4 \pi R a^{\mu}(t, R, \hat{n}) \simeq \frac{1}{u}\left[\frac{1}{4 \pi} \sum_{a=1}^{m} \sum_{\substack{b=1 \\
b \neq a}}^{m} q_{a}^{\prime 2} q_{b}^{\prime} p_{a}^{\prime 2} p_{b}^{\prime 2} \frac{1}{\left\{\left(p_{a}^{\prime} \cdot p_{b}^{\prime}\right)^{2}-p_{a}^{\prime 2} p_{b}^{\prime 2}\right\}^{3 / 2}} \frac{n^{\rho}}{n \cdot p_{a}^{\prime}}\left\{p_{b \rho}^{\prime} p_{a}^{\prime \mu}-p_{a \rho}^{\prime} p_{b}^{\prime \mu}\right\}\right. \\
& \left.-G \sum_{a=1}^{m} \sum_{\substack{b=1 \\
b \neq a}}^{m} q_{a}^{\prime} \frac{n_{\rho}}{n \cdot p_{a}^{\prime}} \frac{p_{a}^{\prime} \cdot p_{b}^{\prime}}{\left\{\left(p_{a}^{\prime} \cdot p_{b}^{\prime}\right)^{2}-p_{a}^{\prime 2} p_{b}^{\prime 2}\right\}^{\frac{3}{2}}}\left(p_{a}^{\prime \mu} p_{b}^{\prime \rho}-p_{a}^{\prime \rho} p_{b}^{\prime \mu}\right)\left\{2\left(p_{a}^{\prime} \cdot p_{b}^{\prime}\right)^{2}-3 p_{a}^{\prime 2} p_{b}^{\prime 2}\right\}\right], \\
& \text { as } u \rightarrow-\infty \text {. }
\end{aligned}
$$

This gives the wave-form of the electromagnetic field at early and late time. The term on the right hand side of the first line gives the constant shift in the vector potential, and is responsible for electromagnetic memory [56-58]. The rest of the terms are tail terms.

\subsection{Electromagnetic contribution to the soft graviton theorem}

We shall now analyze the effect of electromagnetic interaction on the soft graviton theorem. This affects our earlier analysis of soft graviton theorem in two ways. First of all, the Lorentz force on the outgoing and incoming particles changes the particle trajectories, producing an additional contribution to $\widehat{T}^{X \mu \nu}$. Analysis of this follows the same procedure 
that led to (3.32), (4.16), and the final result is given by:

$$
\begin{aligned}
\Delta^{(1)} \widehat{T}^{\mu \nu}(k)= & \sum_{a=1}^{m+n} \sum_{\substack{b=1 \\
b \neq a}}^{m+n} q_{a} q_{b} \int \frac{d^{4} \ell}{(2 \pi)^{4}} \frac{1}{\ell \cdot p_{b}-i \epsilon} G_{r}(\ell) \\
& {\left[\frac{1}{(k-\ell) \cdot p_{a}-i \epsilon} \frac{1}{k \cdot p_{a}-i \epsilon} \frac{1}{\ell \cdot p_{a}+i \epsilon}\left(p_{a} \cdot \ell k \cdot p_{b}-k \cdot \ell p_{a} \cdot p_{b}\right) p_{a}^{\mu} p_{a}^{\nu}\right.} \\
& -\frac{1}{(k-\ell) \cdot p_{a}-i \epsilon} \frac{1}{\ell \cdot p_{a}+i \epsilon}\left(p_{a} \cdot \ell p_{b}^{\mu}-\ell^{\mu} p_{a} \cdot p_{b}\right) p_{a}^{\nu} \\
& \left.-\frac{1}{(k-\ell) \cdot p_{a}-i \epsilon} \frac{1}{\ell \cdot p_{a}+i \epsilon}\left(p_{a} \cdot \ell p_{b}^{\nu}-\ell^{\nu} p_{a} \cdot p_{b}\right) p_{a}^{\mu}\right]
\end{aligned}
$$

Evaluation of this using the method described below (3.32), gives

$$
\begin{aligned}
\Delta^{(1)} \widehat{T}^{\mu \nu}(k)= & \frac{1}{4 \pi} \ln \{L(\omega+i \epsilon)\} \sum_{a=1}^{n} \sum_{\substack{b=1 \\
b \neq a}}^{n} q_{a} q_{b} \frac{1}{\left\{\left(p_{a} \cdot p_{b}\right)^{2}-p_{a}^{2} p_{b}^{2}\right\}^{3 / 2}}\left[\frac{k \cdot p_{b}}{k \cdot p_{a}} p_{a}^{2} p_{b}^{2} p_{a}^{\mu} p_{a}^{\nu}\right. \\
& \left.+p_{b}^{2} p_{a} \cdot p_{b} p_{a}^{\mu} p_{a}^{\nu}-p_{a}^{2} p_{b}^{2}\left(p_{a}^{\mu} p_{b}^{\nu}+p_{a}^{\nu} p_{b}^{\mu}\right)\right] \\
& +\frac{1}{4 \pi} \ln \{L(\omega-i \epsilon)\} \sum_{a=1}^{m} \sum_{\substack{b=1 \\
b \neq a}}^{m} q_{a}^{\prime} q_{b}^{\prime} \frac{1}{\left\{\left(p_{a}^{\prime} \cdot p_{b}^{\prime}\right)^{2}-p_{a}^{\prime 2} p_{b}^{\prime 2}\right\}^{3 / 2}}\left[\frac{k \cdot p_{b}^{\prime}}{k \cdot p_{a}^{\prime}} p_{a}^{\prime 2} p_{b}^{\prime 2} p_{a}^{\prime \mu} p_{a}^{\prime \nu}\right. \\
& \left.+p_{b}^{\prime 2} p_{a}^{\prime} \cdot p_{b}^{\prime} p_{a}^{\prime \mu} p_{a}^{\prime \nu}-p_{a}^{\prime 2} p_{b}^{\prime 2}\left(p_{a}^{\prime \mu} p_{b}^{\prime \nu}+p_{a}^{\prime \nu} p_{b}^{\prime \mu}\right)\right]
\end{aligned}
$$

Second, there is an additional contribution to the stress tensor due to the electromagnetic field. Using the form of the electromagnetic field produced by the charged particle as given in (4.7), this additional contribution takes the form:

$$
\begin{aligned}
\Delta^{(2)} \widehat{T}^{\mu \nu}(k)= & \int d^{4} x e^{-i k \cdot x}\left[\eta^{\mu \rho} \eta^{\nu \sigma} \eta^{\alpha \beta} F_{\rho \alpha} F_{\sigma \beta}-\frac{1}{4} \eta^{\mu \nu} \eta^{\rho \sigma} \eta^{\alpha \beta} F_{\rho \alpha} F_{\sigma \beta}\right] \\
& + \text { higher order terms } \\
\simeq & \sum_{a=1}^{m+n} \sum_{b=1}^{m+n} q_{a} q_{b} \int \frac{d^{4} \ell}{(2 \pi)^{4}} \frac{1}{\ell \cdot p_{b}-i \epsilon} \frac{1}{(k-\ell) \cdot p_{a}-i \epsilon} G_{r}(\ell) G_{r}(k-\ell) \\
& {\left[\ell^{\mu}(k-\ell)^{\nu} p_{a} \cdot p_{b}-\ell^{\mu} p_{a}^{\nu} p_{b} \cdot(k-\ell)-p_{b}^{\mu}(k-\ell)^{\nu} \ell \cdot p_{a}+p_{b}^{\mu} p_{a}^{\nu} \ell .(k-\ell)\right.} \\
& \left.-\frac{1}{2} \eta^{\mu \nu}\left\{\ell \cdot(k-\ell) p_{a} \cdot p_{b}-\ell \cdot p_{a}(k-\ell) \cdot p_{b}\right\}\right] .
\end{aligned}
$$

We shall analyze this by expressing $G_{r}(\ell) G_{r}(k-\ell)$ as in (3.44). The term proportional to $\delta\left(\ell^{2}\right)$ can be analyzed as in appendix $\mathrm{C}$, and one can show that the contribution from this term can be interpreted as the soft graviton emission from electromagnetic wave produced during scattering. Since this is included in the sum over $a$ in the soft factor, we do not need 
to include its contribution. This allows us to replace $G_{r}(\ell)$ by $G_{r}(\ell)^{*}$ in (4.27). Since each term in the numerator of the integrand carries a factor of $\ell$ and a factor of $(k-\ell)$, there is no logarithmic contribution from the $\left|\ell^{\mu}\right| \ll \omega$ and $\left|k^{\mu}-\ell^{\mu}\right| \ll \omega$ regions. Therefore we focus on the $\left|\ell^{\mu}\right| \gg \omega$ region, and analyze the contribution using (3.53), (3.55). The final result is:

$$
\begin{aligned}
\Delta^{(2)} \widehat{T}^{\mu \nu}(k)= & -\frac{1}{4 \pi} \ln (\omega+i \epsilon) \sum_{a=1}^{n} \sum_{\substack{b=1 \\
b \neq a}}^{n} q_{a} q_{b} p_{a} \cdot p_{b} \frac{1}{\left\{\left(p_{a} \cdot p_{b}\right)^{2}-p_{a}^{2} p_{b}^{2}\right\}^{3 / 2}} p_{a}^{\mu}\left(p_{b}^{2} p_{a}^{\nu}-p_{a} \cdot p_{b} p_{b}^{\nu}\right) \\
& -\frac{1}{4 \pi} \ln (\omega-i \epsilon) \sum_{\substack{a=1 \\
m}}^{m} \sum_{\substack{b=1 \\
b \neq a}}^{m} q_{a}^{\prime} q_{b}^{\prime} p_{a}^{\prime} \cdot p_{b}^{\prime} \frac{1}{\left\{\left(p_{a}^{\prime} \cdot p_{b}^{\prime}\right)^{2}-p_{a}^{\prime 2} p_{b}^{\prime 2}\right\}^{3 / 2}} p_{a}^{\prime \mu}\left(p_{b}^{\prime 2} p_{a}^{\prime \nu}-p_{a}^{\prime} \cdot p_{b}^{\prime} p_{b}^{\prime \nu}\right) \\
& -\frac{1}{4 \pi} \ln (\omega+i \epsilon) \sum_{a=1}^{n} \sum_{\substack{b=1 \\
b \neq a}}^{n} q_{a} q_{b} \frac{1}{\left\{\left(p_{a} \cdot p_{b}\right)^{2}-p_{a}^{2} p_{b}^{2}\right\}^{1 / 2}} p_{b}^{\mu} p_{a}^{\nu} \\
& -\frac{1}{4 \pi} \ln (\omega-i \epsilon) \sum_{a=1}^{m} \sum_{\substack{b=1 \\
b \neq a}}^{m} q_{a}^{\prime} q_{b}^{\prime} \frac{1}{\left\{\left(p_{a}^{\prime} \cdot p_{b}^{\prime}\right)^{2}-p_{a}^{\prime 2} p_{b}^{\prime 2}\right\}^{1 / 2}} p_{b}^{\prime \mu} p_{a}^{\prime \nu} .
\end{aligned}
$$

Adding (4.26) and (4.28) we get the net electromagnetic contribution to the soft graviton theorem:

$$
\begin{aligned}
& \Delta^{(1)} \widehat{T}^{\mu \nu}(k)+\Delta^{(2)} \widehat{T}^{\mu \nu}(k) \\
= & \frac{1}{4 \pi} \ln (\omega+i \epsilon) \sum_{\substack{a=1 \\
n}}^{n} \sum_{\substack{b=1 \\
b \neq a}}^{n} q_{a} q_{b} \frac{p_{a}^{2} p_{b}^{2}}{\left\{\left(p_{a} \cdot p_{b}\right)^{2}-p_{a}^{2} p_{b}^{2}\right\}^{3 / 2}}\left[\frac{k \cdot p_{b}}{k \cdot p_{a}} p_{a}^{\mu} p_{a}^{\nu}-p_{a}^{\mu} p_{b}^{\nu}\right] \\
& +\frac{1}{4 \pi} \ln (\omega-i \epsilon) \sum_{\substack{a=1 \\
m}}^{m} \sum_{\substack{b=1 \\
b \neq a}}^{m} q_{a}^{\prime} q_{b}^{\prime} \frac{p_{a}^{\prime 2} p_{b}^{\prime 2}}{\left\{\left(p_{a}^{\prime} \cdot p_{b}^{\prime}\right)^{2}-p_{a}^{\prime 2} p_{b}^{\prime 2}\right\}^{3 / 2}}\left[\frac{k \cdot p_{b}^{\prime}}{k \cdot p_{a}^{\prime}} p_{a}^{\prime \mu} p_{a}^{\prime \nu}-p_{a}^{\prime \mu} p_{b}^{\prime \nu}\right] .
\end{aligned}
$$

From (3.7), (2.6), we can read out the additional contribution to the gravitational waveform at early and late retarded time due to electromagnetic interactions:

$$
\begin{gathered}
\Delta_{\mathrm{em}} e^{\mu \nu} \rightarrow-\frac{G}{2 \pi R u} \sum_{a=1}^{n} \sum_{\substack{b=1 \\
b \neq a}}^{n} q_{a} q_{b} \frac{1}{\left\{\left(p_{a} \cdot p_{b}\right)^{2}-p_{a}^{2} p_{b}^{2}\right\}^{3 / 2}}\left[\frac{k \cdot p_{b}}{k \cdot p_{a}} p_{a}^{2} p_{b}^{2} p_{a}^{\mu} p_{a}^{\nu}-p_{a}^{2} p_{b}^{2} p_{a}^{\mu} p_{b}^{\nu}\right] \\
\text { as } u \rightarrow \infty, \\
\rightarrow \frac{G}{2 \pi R u} \sum_{\substack{a=1 \\
a}}^{m} \sum_{\substack{b=1 \\
b \neq a}}^{m} q_{a}^{\prime} q_{b}^{\prime} \frac{1}{\left\{\left(p_{a}^{\prime} \cdot p_{b}^{\prime}\right)^{2}-p_{a}^{\prime 2} p_{b}^{\prime 2}\right\}^{3 / 2}}\left[\frac{k \cdot p_{b}^{\prime}}{k \cdot p_{a}^{\prime}} p_{a}^{\prime 2} p_{b}^{\prime 2} p_{a}^{\prime \mu} p_{a}^{\prime \nu}-p_{a}^{\prime 2} p_{b}^{\prime 2} p_{a}^{\prime \mu} p_{b}^{\prime \nu}\right] \\
\text { as } u \rightarrow-\infty .
\end{gathered}
$$

\section{New conjectures at the subsubleading order}

Emboldened by the success of soft theorem in correctly predicting the tail of the gravitational wave-form at the subleading order, we shall now propose new conjectures at the 
subsubleading order. It is known that in quantum gravity, the subsubleading soft factors are not universal. Nevertheless there are some universal terms that we could utilize [8]. These are terms that are quadratic in the orbital angular momenta. Our goal will be to make use of these universal terms to arrive at new conjectures on the late and early time tail of gravitational radiation. The non-universal terms do not involve orbital angular momenta and therefore do not have logarithmic divergences. Hence they will not affect our analysis.

Using the relation between quantum soft factors and classical gravitational wave-forms derived in [15], and ignoring the non-universal terms, we can write down the following form of the gravitational wave-form to subsubleading order: ${ }^{8}$

$$
\begin{aligned}
\widetilde{e}^{\mu \nu}(\omega, \vec{x})= & \frac{2 G}{R} e^{i \omega R} \exp \left[-2 i G \ln \{R(\omega+i \epsilon)\} \sum_{b=1}^{n} p_{b} \cdot k\right] \\
& \times \sum_{a=1}^{m+n}\left[-i \frac{p_{a}^{\mu} p_{a}^{\nu}}{p_{a} \cdot k}-\frac{1}{p_{a} \cdot k} J_{a}^{\rho(\nu} p_{a}^{\mu)} k_{\rho}+\frac{i}{2} \frac{1}{p_{a} \cdot k} k_{\rho} k_{\sigma} J_{a}^{\mu \rho} J_{a}^{\nu \sigma}\right],
\end{aligned}
$$

where $k$ has been defined in (A.8) and $J_{a}^{\rho \sigma}$ is the sum of the orbital and spin angular momenta of the $a$-th external particle:

$$
J_{a}^{\rho \sigma}=X_{a}^{\rho} p_{a}^{\sigma}-X_{a}^{\sigma} p_{a}^{\rho}+\Sigma_{a}^{\rho \sigma} .
$$

The second term in the last line of (5.1) differs by a sign from the expressions used e.g. in [15]. This can be traced to the fact that in [15] we treated the charges / momenta / angular momenta carried by ingoing particles as positive and of the outgoing particles as negative, whereas here we are following the opposite convention. Following (3.12), the spin $\Sigma_{a}^{\prime \mu \nu}$ for incoming particles are given by:

$$
\Sigma_{a}^{\prime \mu \nu}=-\Sigma_{a+n}^{\mu \nu} \text { for } 1 \leq a \leq m .
$$

The phase factor $\exp \left[-2 i G \ln \{R(\omega+i \epsilon)\} \sum_{b=1}^{n} p_{b} . k\right]$ in (5.1) is not determined by soft theorem, but is determined by independent computation [33, 47, 59], and is consistent with the term in the last line of (3.59). Due to the long range gravitational force between the outgoing / incoming particles, $X_{a}^{\rho}$ has logarithmic corrections at late / early time [17], leading to $[18]$

$$
\begin{aligned}
X_{a}^{\rho} p_{a}^{\sigma}-X_{a}^{\sigma} p_{a}^{\rho}= & -G \sum_{\substack{b \neq a \\
\eta_{a} \eta_{b}=1}} \ln \left|\sigma_{a}\right| \frac{p_{b} \cdot p_{a}}{\left\{\left(p_{b} \cdot p_{a}\right)^{2}-p_{a}^{2} p_{b}^{2}\right\}^{3 / 2}}\left(p_{b}^{\rho} p_{a}^{\sigma}-p_{b}^{\sigma} p_{a}^{\rho}\right)\left\{2\left(p_{b} \cdot p_{a}\right)^{2}-3 p_{a}^{2} p_{b}^{2}\right\} \\
& +\left(r_{a}^{\rho} p_{a}^{\sigma}-r_{a}^{\sigma} p_{a}^{\rho}\right)
\end{aligned}
$$

where $\sigma_{a}$ denotes the proper time of the $a$-th particle, and $r_{a}$ is the constant that appeared in (3.23). The contribution proportional to $\ln \left|\sigma_{a}\right|$ arises from the correction term $Y_{a}$ in (3.23). The conjecture of $[17,18]$ was that the $\ln \left|\sigma_{a}\right|$ factor should be replaced by

\footnotetext{
${ }^{8} \mathrm{~A}$ non-trivial test of this formula for the scattering of massless particles can be found in [34].
} 
$\ln \omega^{-1}$ in (5.1). After including the $i \epsilon$ prescription described in this paper, this conjecture translates to the rule that in $(5.1), J_{a}^{\rho \sigma}$ should be replaced by:

$$
\begin{aligned}
J_{a}^{\rho \sigma}= & G \sum_{\substack{b \neq a \\
\eta_{a} \eta_{b}=1}} \ln \left(\omega+i \epsilon \eta_{a}\right) \frac{p_{b} \cdot p_{a}}{\left\{\left(p_{b} \cdot p_{a}\right)^{2}-p_{a}^{2} p_{b}^{2}\right\}^{3 / 2}}\left(p_{b}^{\rho} p_{a}^{\sigma}-p_{b}^{\sigma} p_{a}^{\rho}\right)\left\{2\left(p_{b} \cdot p_{a}\right)^{2}-3 p_{a}^{2} p_{b}^{2}\right\} \\
& +\left(r_{a}^{\rho} p_{a}^{\sigma}-r_{a}^{\sigma} p_{a}^{\rho}\right)+\Sigma_{a}^{\rho \sigma} .
\end{aligned}
$$

We now substitute (5.5) into (5.1) and expand the expression in powers of $\omega$, including the $\exp \left[-2 i G \ln \{R(\omega+i \epsilon)\} \sum_{b=1}^{n} p_{b} . k\right]$ term. Terms proportional to $\ln (\omega \pm i \epsilon)$ reproduce correctly (3.59). We shall focus on terms proportional to $\omega(\ln \omega)^{2}$. In the $\omega$ space these terms are subdominant compared to the order $\omega^{0}$ terms that we have left out from the subleading terms in the gravitational wave-form. However after Fourier transformation, polynomials in $\omega$ produce local terms in time, while terms involving $\ln \omega$ produce tail terms that survive at late and early retarded time. Therefore the corrections to (3.61) at late and early time will be dominated by the terms proportional to $\omega(\ln \omega)^{2}$ in the expression for $\widetilde{e}^{\mu \nu}$. The order $\omega^{0}$ terms may have other observational signature, e.g. the spin memory discussed in [60].

Expanding (5.1) in powers of $\omega$, with $J_{a}^{\rho \sigma}$ given by (5.5), we get the corrections proportional to $\omega(\ln \omega)^{2}$. These take the form:

$$
\begin{aligned}
\Delta_{\text {subsubleading }} \widetilde{e}^{\mu \nu}= & e^{i \omega R-2 i \omega G \ln R \sum_{d=1}^{n} p_{d} \cdot n} i \frac{G^{3}}{R} \omega \\
& {\left[4\{\ln (\omega+i \epsilon)\}^{2} \sum_{b=1}^{n} p_{b} \cdot n \sum_{c=1}^{n} p_{c} \cdot n \sum_{a=1}^{m+n} \frac{p_{a}^{\mu} p_{a}^{\nu}}{p_{a} \cdot n}\right.} \\
& +4 \ln (\omega+i \epsilon) \sum_{c=1}^{n} p_{c} \cdot n \sum_{a=1}^{m+n} \sum_{\substack{b=1 \\
b \neq n}}^{m+n} \ln \left(\omega+i \epsilon \eta_{a}\right) \frac{1}{p_{a} \cdot n} \frac{p_{a} \cdot p_{b}}{\left\{\left(p_{a} \cdot p_{b}\right)^{2}-p_{a}^{2} p_{b}^{2}\right\}^{3 / 2}} \\
& \left\{2\left(p_{a} \cdot p_{b}\right)^{2}-3 p_{a}^{2} p_{b}^{2}\right\}\left\{n \cdot p_{b} p_{a}^{\mu} p_{a}^{\nu}-n \cdot p_{a} p_{a}^{\mu} p_{b}^{\nu}\right\} \\
& +\sum_{a=1}^{m+n} \sum_{b=1}^{m+n} \sum_{c=1}^{m+n}\left\{\ln \left(\omega+i \epsilon \eta_{a}\right)\right\}^{2} \frac{1}{p_{a} \cdot n} \frac{p_{a} \cdot p_{b}}{\left\{\left(p_{a} \cdot p_{b}\right)^{2}-p_{a}^{2} p_{b}^{2}\right\}^{3 / 2}} \\
& \left\{2\left(p_{a} \cdot p_{b}\right)^{2}-3 p_{a}^{2} p_{b}^{2}\right\} \frac{\eta_{a}}{\left\{\left(p_{a} \cdot p_{c}\right)^{2}-p_{a}^{2} p_{c}^{2}\right\}^{3 / 2}}\left\{2\left(p_{a} \cdot p_{c}\right)^{2}-3 p_{a}^{2} p_{c}^{2}\right\} \\
& \left.\left\{n \cdot p_{b} p_{a}^{\mu}-n \cdot p_{a} p_{b}^{\mu}\right\}\left\{n \cdot p_{c} p_{a}^{\nu}-n \cdot p_{a} p_{c}^{\nu}\right\}\right] .
\end{aligned}
$$

Using (2.7), (2.8), we now get,

$$
\Delta_{\text {subsubleading }} e_{\mu \nu} \rightarrow\left\{\begin{array}{lll}
u^{-2} \ln |u| F_{\mu \nu} & \text { as } & u \rightarrow \infty \\
u^{-2} \ln |u| G_{\mu \nu} & \text { as } & u \rightarrow-\infty
\end{array}\right.
$$


where

$$
\begin{aligned}
& F^{\mu \nu}=2 \frac{G^{3}}{R}\left[4 \sum_{b=1}^{n} p_{b} \cdot n \sum_{c=1}^{n} p_{c} \cdot n\left\{\sum_{a=1}^{n} \frac{p_{a}^{\mu} p_{a}^{\nu}}{p_{a} \cdot n}-\sum_{a=1}^{m} \frac{p_{a}^{\prime \mu} p_{a}^{\prime \nu}}{p_{a}^{\prime} \cdot n}\right\}\right. \\
& +4 \sum_{c=1}^{n} p_{c} \cdot n \sum_{a=1}^{n} \sum_{\substack{b=1 \\
b \neq a}}^{n} \frac{1}{p_{a} \cdot n} \frac{p_{a} \cdot p_{b}}{\left\{\left(p_{a} \cdot p_{b}\right)^{2}-p_{a}^{2} p_{b}^{2}\right\}^{3 / 2}}\left\{2\left(p_{a} \cdot p_{b}\right)^{2}-3 p_{a}^{2} p_{b}^{2}\right\}\left\{n \cdot p_{b} p_{a}^{\mu} p_{a}^{\nu}-n \cdot p_{a} p_{a}^{\mu} p_{b}^{\nu}\right\} \\
& +2 \sum_{c=1}^{n} p_{c} \cdot n \sum_{a=1}^{m} \sum_{\substack{b=1 \\
b \neq a}}^{m} \frac{1}{p_{a}^{\prime} \cdot n} \frac{p_{a}^{\prime} \cdot p_{b}^{\prime}}{\left\{\left(p_{a}^{\prime} \cdot p_{b}^{\prime}\right)^{2}-p_{a}^{\prime 2} p_{b}^{\prime 2}\right\}^{3 / 2}}\left\{2\left(p_{a}^{\prime} \cdot p_{b}^{\prime}\right)^{2}-3 p_{a}^{\prime 2} p_{b}^{\prime 2}\right\}\left\{n \cdot p_{b}^{\prime} p_{a}^{\prime \mu} p_{a}^{\prime \nu}-n \cdot p_{a}^{\prime} p_{a}^{\prime \mu} p_{b}^{\prime \nu}\right\} \\
& +\sum_{a=1}^{n} \sum_{\substack{b=1 \\
b \neq a}}^{n} \sum_{c=1}^{n} \frac{1}{p_{a} \cdot n} \frac{p_{a} \cdot p_{b}}{\left\{\left(p_{a} \cdot p_{b}\right)^{2}-p_{a}^{2} p_{b}^{2}\right\}^{3 / 2}}\left\{2\left(p_{a} \cdot p_{b}\right)^{2}-3 p_{a}^{2} p_{b}^{2}\right\} \frac{p_{a} \cdot p_{c}}{\left\{\left(p_{a} \cdot p_{c}\right)^{2}-p_{a}^{2} p_{c}^{2}\right\}^{3 / 2}} \\
& \left.\quad\left\{2\left(p_{a} \cdot p_{c}\right)^{2}-3 p_{a}^{2} p_{c}^{2}\right\}\left\{n \cdot p_{b} p_{a}^{\mu}-n \cdot p_{a} p_{b}^{\mu}\right\}\left\{n \cdot p_{c} p_{a}^{\nu}-n \cdot p_{a} p_{c}^{\nu}\right\}\right],
\end{aligned}
$$

and

$$
\begin{aligned}
G^{\mu \nu}= & -2 \frac{G^{3}}{R}\left[2 \sum_{c=1}^{n} p_{c} \cdot n \sum_{a=1}^{m} \sum_{\substack{b=1 \\
b \neq a}}^{m} \frac{1}{p_{a}^{\prime} \cdot n} \frac{p_{a}^{\prime} \cdot p_{b}^{\prime}}{\left\{\left(p_{a}^{\prime} \cdot p_{b}^{\prime}\right)^{2}-p_{a}^{\prime 2} p_{b}^{\prime 2}\right\}^{3 / 2}}\left\{2\left(p_{a}^{\prime} \cdot p_{b}^{\prime}\right)^{2}-3 p_{a}^{\prime 2} p_{b}^{\prime 2}\right\}\right. \\
& \left\{n \cdot p_{b}^{\prime} p_{a}^{\prime \mu} p_{a}^{\prime \nu}-n \cdot p_{a}^{\prime} p_{a}^{\prime \mu} p_{b}^{\prime \nu}\right\} \\
& -\sum_{\substack{a=1 \\
m}}^{m} \sum_{\substack{b=1 \\
b \neq a}}^{m} \sum_{\substack{c=1 \\
c \neq a}}^{m} \frac{1}{p_{a}^{\prime} \cdot n} \frac{p_{a}^{\prime} \cdot p_{b}^{\prime}}{\left\{\left(p_{a}^{\prime} \cdot p_{b}^{\prime}\right)^{2}-p_{a}^{\prime 2} p_{b}^{\prime 2}\right\}^{3 / 2}}\left\{2\left(p_{a}^{\prime} \cdot p_{b}^{\prime}\right)^{2}-3 p_{a}^{\prime 2} p_{b}^{\prime 2}\right\} \frac{p_{a}^{\prime} \cdot p_{c}^{\prime}}{\left\{\left(p_{a}^{\prime} \cdot p_{c}^{\prime}\right)^{2}-p_{a}^{\prime 2} p_{c}^{\prime 2}\right\}^{3 / 2}} \\
& \left.\left\{2\left(p_{a}^{\prime} \cdot p_{c}^{\prime}\right)^{2}-3 p_{a}^{\prime 2} p_{c}^{\prime 2}\right\}\left\{n \cdot p_{b}^{\prime} p_{a}^{\prime \mu}-n \cdot p_{a}^{\prime} p_{b}^{\prime \mu}\right\}\left\{n \cdot p_{c}^{\prime} p_{a}^{\prime \nu}-n \cdot p_{a}^{\prime} p_{c}^{\prime \nu}\right\}\right] .
\end{aligned}
$$

One can check that in case of binary black hole merger, regarded as a process in which a single massive object decays into a massive object and many massless particles (gravitational waves), $F^{\mu \nu}$ and $G^{\mu \nu}$ vanish.

One could attempt to prove these results following the same procedure employed in this paper. For this we need to iteratively solve the equations of motion to one order higher than what has been done in this paper. Terms of order $\omega \ln \omega$ would also receive contributions from the expansion of the factors of $e^{i k . r_{a}}$ in various expressions in this paper, e.g. in (3.32), to first order in $k$, and will therefore depend on the additional data $\left\{r_{a}\right\}$. However the $\omega(\ln \omega)^{2}$ terms given in (5.6) do not suffer from any such ambiguity.

It may be possible to find higher order generalization of these results using the exponentiated soft factor discussed in [45, 61].

\section{$6 \quad$ Numerical estimate}

Before concluding the paper, we shall give estimates of the coefficient of the $1 / u$ term in (1.4) in some actual physical processes.

1. Hypervelocity stars: When a binary star system comes close to the supermassive black hole at the center of the milky way, often one of them gets captured by the 
black hole and the other escapes with a high velocity, producing a hypervelocity star [62]. This can be taken as a two body decay of a single bound object. Using the mass of the central black hole to be of order $10^{5} M_{\odot}$, the mass of the hypervelocity star to be of order $M_{\odot}$, its velocity to be of order $3 \times 10^{-3} c$, and the distance of the earth from the galactic center to be of order 25000 light-years, one can estimate the coefficient of the $1 / u$ term in (1.4) to be of order $10^{-22}$ days. The minimum value of $u$ needed for (1.4) to hold - namely when the kinetic energy dominates the gravitational potential energy [19] — in this case is about a day. This gives a strain of order $10^{-22}$, which is at the edge of the detection sensitivity of the future space-based gravitational wave detectors.

2. Core collapse supernova: This case was already discussed in [19]. During this process the residual neutron star often gets a high velocity kick which could be of the order of $1000 \mathrm{~km} / \mathrm{sec}$, balanced by ejected matter in opposite direction at a speed up to 5000 $\mathrm{km} / \mathrm{sec}$. [63]. Taking the neutron star to have a mass of order $M_{\odot}$ and the supernova to be in our galaxy so that its distance from the earth is of the order of $10^{5}$ light years, the coefficient of the $1 / u$ term was computed to be of order $10^{-22} \mathrm{sec}$. The minimum value of $u$ for which the asymptotic formula holds was found to be of order 1 sec. Therefore the strain at this time will be of order $10^{-22}$, which is at the edge of the detection limit of the current gravitational wave detectors.

3. Binary black hole merger: As already pointed out, for binary black hole merger, the coefficient of the $1 / u$ term vanishes due to cancellation between various terms. However the individual terms in (1.4) have the same order of magnitude as the memory effect when the asymptotic formula can be trusted, which is of the order of the light crossing time of the horizon. Therefore the observation of the memory effect without observation of the $1 / u$ tail is a prediction of general theory of relativity that can be tested in future gravitational wave experiments.

4. Bullet cluster: The bullet cluster [64] consists of a pair of galaxy clusters, each with mass of about $10^{14} M_{\odot}$ [65], passing through each other at a speed of about $10^{-2} c$. The system is situated at a distance of about $4 \times 10^{9}$ light-years from the earth. Using this data we get the coefficient of the $1 / u$ term in (1.4) to be of the order of $10^{-6}$ year. The retarded time $u$ for this system, - the time that has elapsed since the centers of the two clusters passed each other, - is about $1.5 \times 10^{8}$ years. This gives the current value of the strain produced by the bullet cluster in our neighborhood to be about $10^{-14}$. While this is much larger than the sensitivity of the current gravitational wave detectors, what the latter detect is not the strain but the change in the strain - more precisely its second $u$ derivative that enters the expression for the Riemann tensor. For the bullet cluster this is too small an effect to be observed by the conventional gravitational wave detectors. 


\section{Acknowledgments}

We would like to thank Alok Laddha for collaboration at the initial stages of this work, many useful discussions, and comments on an earlier version of the manuscript. We would also like to thank Alfredo Guevara, Dileep Jatkar and Justin Vines for useful discussions. The work of A.S. was supported in part by the J.C. Bose fellowship of the Department of Science and Technology, India and the Infosys chair professorship.

\section{A Derivation of some useful mathematical results}

In this appendix we give the derivation of the results described in section 2 .

\section{A.1 Radiative field at large distance}

Let us consider a differential equation of the form:

$$
\square F(x)=-j(x), \quad \square \equiv \eta^{\alpha \beta} \partial_{\alpha} \partial_{\beta},
$$

where $j(x)$ is some given function. The retarded solution to this equation is given by

$$
F(x)=-\int d^{4} y G_{r}(x, y) j(y),
$$

where $G_{r}(x, y)$ is the retarded Green's function:

$$
G_{r}(x, y)=\int \frac{d^{4} \ell}{(2 \pi)^{4}} e^{i \ell .(x-y)} \frac{1}{\left(\ell^{0}+i \epsilon\right)^{2}-\vec{\ell}^{2}} .
$$

Using (2.1) we get

$$
\widetilde{F}(\omega, \vec{x})=-\int d^{4} y j(y) \int \frac{d^{3} \ell}{(2 \pi)^{3}} e^{i \omega y^{0}+i \vec{\ell} \cdot(\vec{x}-\vec{y})} \frac{1}{(\omega+i \epsilon)^{2}-\vec{\ell}^{2}} .
$$

For large $|\vec{x}|$, we can evaluate this integral using a saddle point approximation as follows [15]. Defining $\vec{\ell}_{\|}$and $\vec{\ell}_{\perp}$ as components of $\vec{\ell}$ along $\vec{x}-\vec{y}$ and transverse to $\vec{x}-\vec{y}$ respectively, we get

$$
\widetilde{F}(\omega, \vec{x})=-\int d^{4} y j(y) \int \frac{d^{2} \ell_{\perp}}{(2 \pi)^{2}} \frac{d \ell_{\|}}{2 \pi} e^{i \omega y^{0}+i \ell_{\|}|\vec{x}-\vec{y}|} \frac{1}{(\omega+i \epsilon)^{2}-\ell_{\|}^{2}-\overrightarrow{\ell_{\perp}^{2}}} .
$$

First consider the case $\omega>0$. We now close the $\ell_{\|}$integration contour in the upper half plane, picking up residue at the pole at $\sqrt{(\omega+i \epsilon)^{2}-\vec{\ell}_{\perp}^{2}}$. This gives

$$
\widetilde{F}(\omega, \vec{x})=i \int d^{4} y j(y) \int \frac{d^{2} \ell_{\perp}}{(2 \pi)^{2}} e^{i \omega y^{0}+i|\vec{x}-\vec{y}| \sqrt{(\omega+i \epsilon)^{2}-\vec{\ell}_{\perp}^{2}}} \frac{1}{2 \sqrt{(\omega+i \epsilon)^{2}-\vec{\ell}_{\perp}^{2}}} .
$$

For large $|\vec{x}-\vec{y}|$ the exponent is a rapidly varying function of $\vec{\ell}_{\perp}$ and therefore we can carry out the integration over $\vec{\ell}_{\perp}$ using saddle point approximation. The saddle point is located 
at $\vec{\ell}_{\perp}=0$. Expanding the exponent to order $\vec{\ell}_{\perp}^{2}$ and carrying out gaussian integration over $\vec{\ell}_{\perp}$ we get:

$$
\widetilde{F}(\omega, \vec{x})=i \int d^{4} y j(y) e^{i \omega y^{0}+i(\omega+i \epsilon)|\vec{x}-\vec{y}|} \frac{\omega+i \epsilon}{2 \pi i|\vec{x}-\vec{y}|} \frac{1}{2(\omega+i \epsilon)} \simeq \frac{1}{4 \pi R} e^{i \omega R} \int d^{4} y e^{-i k \cdot y} j(y),
$$

where we have made the approximation $|\vec{x}| \gg|\vec{y}|$, and,

$$
k \equiv \omega(1, \hat{n}), \quad \hat{n} \equiv \vec{x} /|\vec{x}|, \quad R \equiv|\vec{x}| .
$$

A similar analysis can be carried out for $\omega<0$, leading to the same final expression. Using (2.1), eq. (A.7) may be written as

$$
\widetilde{F}(\omega, \vec{x}) \simeq \frac{1}{4 \pi R} e^{i \omega R} \widehat{j}(k) .
$$

This is a known formula (see e.g. [36]), but the derivation given above also gives its limitations. In arriving at the right hand side of (A.7) we used the approximation $|\vec{x}| \gg|\vec{y}|$. Therefore in the integration over $\vec{y}$ there is a natural infrared cut-off given by $|\vec{x}|=R$. If the $y$ integral is convergent then there is no need of such a cut-off, but in case the $y$ integral diverges from the large $y$ region, we need to explicitly impose the cut-off. We can implement the cut-off by putting a cut-off on $y^{0}$, since typically the source $j(y)$ will have support inside the light-cone $|\vec{y}| \leq\left|y^{0}\right|$ for large $y$. For example, for positive $y^{0}$ we can implement the infrared cut-off by adding to $k^{0}$ an imaginary part $i \Lambda R^{-1}$ for some fixed number $\Lambda$. In that case for $y^{0} \ll R / \Lambda$ this additional factor has no effect on (A.7), but for $y^{0} \gg R / \Lambda$ there is an exponential suppression factor that cuts off the integration over $y$. For negative $y^{0}$ the corresponding modification of $k$ corresponds to adding an imaginary part $-i \Lambda R^{-1}$ to $k^{0}$.

\section{A.2 Late and early time behaviour from Fourier transformation}

In our analysis we shall encounter functions $\widetilde{F}(\omega, \vec{x})$ that are non-analytic as $\omega \rightarrow 0$, having singularities either of the form $1 / \omega$ or of the form $\ln \omega$. On general grounds we expect these singular small $\omega$ behaviour to be related to the behaviour of $F(t, \vec{x})$ as $t \rightarrow \pm \infty$. We shall now determine the precise correspondence between the small $\omega$ behaviour of $\widetilde{F}(\omega, \vec{x})$ and large $|t|$ behaviour of $F(t, \vec{x})$. Since the analysis will be carried out at fixed $\vec{x}$, we shall not display the $\vec{x}$ dependence of various quantities in subsequent discussions.

First we shall consider singularities of the form $1 / \omega$ for small $\omega$. For this consider a function of the form:

$$
\widetilde{F}(\omega)=C e^{i \omega \phi} \frac{1}{\omega} f(\omega) .
$$

Here $C$ and $\phi$ are constants that could depend on $\vec{x}$. $f(\omega)$ is a function of $\omega$ that is smooth at $\omega=0$ with $f(0)=1$ and falls off sufficiently fast as $\omega \rightarrow \infty$ so as to make the Fourier integral over $\omega$ well defined. Our final result will not depend on $f(\omega)$, but for definiteness we shall choose

$$
f(\omega)=\frac{1}{\omega^{2}+1}
$$


This gives

$$
F(t)=\int \frac{d \omega}{2 \pi} e^{-i \omega t} \widetilde{F}(\omega)=C \int \frac{d \omega}{2 \pi} e^{-i \omega u} \frac{1}{\omega} f(\omega), \quad u \equiv t-\phi .
$$

In order to define the integral around $\omega=0$, we need to choose an appropriate $i \epsilon$ prescription. However since $1 /(\omega+i \epsilon)$ and $1 /(\omega-i \epsilon)$ differ by a term proportional to $\delta(\omega)$, whose Fourier transform is a $u$ independent constant, the difference will not be of interest to us. For definiteness, we shall work with $1 /(\omega+i \epsilon)$. Then we have

$$
F(t)=C \frac{1}{2 \pi} \int d \omega e^{-i \omega u} \frac{1}{\omega+i \epsilon} f(\omega)=-i C H(u)+\mathcal{O}\left(e^{-u}\right),
$$

where $H$ is the Heaviside step function. This result is obtained by closing the contour in the lower (upper) half plane for positive (negative) $u$, and picking up the residues at the poles. The order $e^{-u}$ contribution comes from the residues at the poles of $f(\omega)$. The step function $H(u)$ gives a jump in $e_{\mu \nu}$ between $u \rightarrow-\infty$ and $u \rightarrow \infty$, leading to the memory effect $[22-25]$.

Let us now turn to the Fourier transform of the logarithmic terms. We consider functions of the form:

$$
\widetilde{F}(\omega)=C e^{i \omega \phi} \ln \omega f(\omega) .
$$

Again we need to consider the different $i \epsilon$ prescriptions, and this time the difference between the two choices is not trivial. Therefore we consider ${ }^{9}$

$$
F_{ \pm}(t)=C \int \frac{d \omega}{2 \pi} e^{-i \omega t} e^{i \omega \phi} \ln (\omega \pm i \epsilon) f(\omega)=C \int \frac{d \omega}{2 \pi} e^{-i \omega u} \ln (\omega \pm i \epsilon) f(\omega) .
$$

For $u>0$ we can close the contour in the lower half plane. In this case $F_{-}$gets contribution only from the poles of $f(\omega)$ and therefore is suppressed by factors of $e^{-u}$. Similarly for $u<0, F_{+}$is suppressed by powers of $e^{-u}$. Furthermore, using $\ln (\omega+i \epsilon)=\ln (\omega-i \epsilon)+$ $2 \pi i H(-\omega)$, we have

$$
F_{+}-F_{-}=i C \int_{-\infty}^{0} d \omega e^{-i \omega u} f(\omega) \simeq-\frac{C}{u}, \quad \text { for } \quad u \rightarrow \pm \infty .
$$

Using these results we get

$$
\begin{aligned}
& F_{+} \equiv C \int \frac{d \omega}{2 \pi} e^{-i \omega u} \ln (\omega+i \epsilon) f(\omega) \rightarrow\left\{\begin{array}{l}
-\frac{C}{u} \text { for } u \rightarrow \infty \\
0 \text { for } u \rightarrow-\infty
\end{array}\right. \\
& F_{-} \equiv C \int \frac{d \omega}{2 \pi} e^{-i \omega u} \ln (\omega-i \epsilon) f(\omega) \rightarrow\left\{\begin{array}{l}
0 \text { for } u \rightarrow \infty, \\
\frac{C}{u} \text { for } u \rightarrow-\infty .
\end{array}\right.
\end{aligned}
$$

\footnotetext{
${ }^{9}$ If $F(t)$ is real, we must have from $(2.1) \widehat{F}(\omega)=\widehat{F}(-\omega)^{*}$. Now since $\ln (-\omega+i \epsilon)^{*}=\ln (-\omega-i \epsilon)=$ $\ln (\omega+i \epsilon)-i \pi$, we see that $\ln (\omega+i \epsilon)$ is not a good candidate for $\widetilde{F}(\omega)$. This can be rectified by averaging over $\ln (\omega+i \epsilon)$ and $\ln (-\omega-i \epsilon)$. However since the two differ by a constant, whose Fourier transform, being proportional to $\delta(u)$, does not affect the behavior at large $|u|$, we shall ignore this complication. A similar remark holds for $\ln (\omega-i \epsilon)$.
} 
Next we shall consider the integrals:

$$
G_{ \pm} \equiv C \int \frac{d \omega}{2 \pi} e^{-i \omega u} \omega\{\ln (\omega \pm i \epsilon)\}^{2} f(\omega) .
$$

As before, $G_{+}$vanishes for large negative $u$ and $G_{-}$vanishes for large positive $u$ up to exponentially suppressed corrections. Furthermore we have

$$
\begin{aligned}
G_{+}-G_{-} & =4 \pi i C \int_{-\infty}^{0} \frac{d \omega}{2 \pi} e^{-i \omega u} \omega\{\ln (\omega-i \epsilon)+i \pi\} f(\omega) \\
& =-4 \pi C \frac{d}{d u} \int_{-\infty}^{0} \frac{d \omega}{2 \pi} e^{-i \omega u}\{\ln (\omega-i \epsilon)+i \pi\} f(\omega) .
\end{aligned}
$$

Changing integration variable to $v=\omega u$ we can express this as

$$
\begin{aligned}
G_{+}-G_{-} & =-2 C \frac{d}{d u}\left[u^{-1} \int_{-\infty \times \operatorname{sign} u}^{0} d v e^{-i v}\{\ln (v-i \epsilon)-\ln u+i \pi\} f(v / u)\right] \\
& =-2 C \frac{d}{d u}\left[-i u^{-1} \ln u+\mathcal{O}\left(u^{-1}\right)\right]=-2 i C u^{-2} \ln |u|+\mathcal{O}\left(u^{-2}\right) .
\end{aligned}
$$

This gives

$$
\begin{aligned}
& G_{+} \equiv C \int \frac{d \omega}{2 \pi} e^{-i \omega u} \omega\{\ln (\omega+i \epsilon)\}^{2} f(\omega) \rightarrow\left\{\begin{array}{l}
-2 i C u^{-2} \ln |u| \text { for } u \rightarrow \infty, \\
0 \quad \text { for } u \rightarrow-\infty,
\end{array}\right. \\
& G_{-} \equiv C \int \frac{d \omega}{2 \pi} e^{-i \omega u} \omega\{\ln (\omega-i \epsilon)\}^{2} f(\omega) \rightarrow \begin{cases}0 & \text { for } u \rightarrow \infty, \\
2 i C u^{-2} \ln |u| & \text { for } u \rightarrow-\infty,\end{cases}
\end{aligned}
$$

up to corrections of order $u^{-2}$.

Finally we consider the integral:

$$
H \equiv C \int \frac{d \omega}{2 \pi} e^{-i \omega u} \omega \ln (\omega+i \epsilon) \ln (\omega-i \epsilon) f(\omega) .
$$

For evaluating this we use the result:

$$
\begin{aligned}
G_{+}+G_{-}-2 H & =C \int \frac{d \omega}{2 \pi} e^{-i \omega u} \omega\{\ln (\omega+i \epsilon)-\ln (\omega-i \epsilon)\}^{2} f(\omega) \\
& =-2 \pi C \int_{-\infty}^{0} d \omega e^{-i \omega u} \omega f(\omega)=\mathcal{O}\left(u^{-2}\right) .
\end{aligned}
$$

Using (2.7) we now get:

$$
H \equiv C \int \frac{d \omega}{2 \pi} e^{-i \omega u} \omega \ln (\omega+i \epsilon) \ln (\omega-i \epsilon) f(\omega) \rightarrow\left\{\begin{array}{l}
-i C u^{-2} \ln |u| \text { for } u \rightarrow \infty \\
i C u^{-2} \ln |u| \text { for } u \rightarrow-\infty
\end{array}\right.
$$




\section{B Evaluation of some integrals}

In this appendix we shall review the evaluation of several integrals following [18].

We begin with the integral

$$
J_{a b}=\int \frac{d^{4} \ell}{(2 \pi)^{4}} \frac{1}{\ell \cdot p_{b}-i \epsilon} G_{r}(\ell) \frac{1}{(\ell-k) \cdot p_{a}+i \epsilon} \simeq \int \frac{d^{4} \ell}{(2 \pi)^{4}} \frac{1}{\ell \cdot p_{b}-i \epsilon} \frac{1}{\left(\ell^{0}+i \epsilon\right)^{2}-\overrightarrow{\ell^{2}}} \frac{1}{\ell \cdot p_{a}+i \epsilon},
$$

with the understanding that the integration over $\ell$ is restricted to the region $L^{-1} \gg\left|\vec{\ell}_{\perp}\right| \gg$ $\omega$. Simple power counting, together with the contour deformation arguments given in the paragraph containing (3.33), then shows that the logarithmic contribution can come only from the region $\left|\ell^{\mu}\right| \sim\left|\ell_{\perp}\right|$ for all $\mu$. However, since the $\ell^{0}$ and $\ell^{3}$ integrals converge for fixed $\ell_{\perp}$, we shall take the range of these integrals to be unrestricted.

First consider the case where $a$ represents an incoming particle and $b$ represents an outgoing particle. In this case $p_{a}^{0}=-p_{a-n}^{\prime 0}<0$ and $p_{b}^{0}>0$. In the $\ell^{0}$ plane the poles of $G_{r}(\ell)$ are in the lower half plane, and the zeroes of $\ell \cdot p_{a}+i \epsilon$ and $\ell \cdot p_{b}-i \epsilon$ are also in the lower half plane. Therefore we can close the $\ell^{0}$ integration contour in the upper half plane and the integral vanishes.

If $a$ represents an outgoing particle and $b$ represents an incoming particle, then the zeroes of $\ell . p_{a}+i \epsilon$ and $\ell . p_{b}-i \epsilon$ are in the upper half plane. Therefore the $\ell^{0}$ integral does not vanish automatically. We can evaluate this by choosing a special frame in which $p_{a}$ and $-p_{b}$ both carry spatial momenta along the third direction, with velocities $\beta_{a}$ and $\beta_{b}$ respectively. Then the integral takes the form:

$$
\begin{aligned}
& \int \frac{d^{4} \ell}{(2 \pi)^{4}} \frac{1}{p_{a}^{0} p_{b}^{0}} \frac{1}{\ell^{0}-\beta_{a} \ell^{3}-i \epsilon_{1}} \frac{1}{\ell^{0}-\beta_{b} \ell^{3}-i \epsilon_{2}} \frac{1}{\left(\ell^{0}+i \epsilon\right)^{2}-\vec{\ell}^{2}} \\
= & i \int \frac{d^{3} \ell}{(2 \pi)^{3}} \frac{1}{p_{a}^{0} p_{b}^{0}} \frac{1}{\left(\beta_{a}-\beta_{b}\right) \ell^{3}+i\left(\epsilon_{1}-\epsilon_{2}\right)}\left[-\frac{1}{\left(1-\beta_{a}^{2}\right)\left(\ell^{3}\right)^{2}+\vec{\ell}_{\perp}^{2}}+\frac{1}{\left(1-\beta_{b}^{2}\right)\left(\ell^{3}\right)^{2}+\vec{\ell}_{\perp}^{2}}\right],
\end{aligned}
$$

where $\ell_{\perp} \equiv\left(\ell^{1}, \ell^{2}\right)$. In the second step we have evaluated the $\ell^{0}$ integral by closing its integration contour in the upper half plane. Since $\beta_{a}, \beta_{b} \leq 1$, the denominators of the terms inside the square bracket never vanish and we have dropped the $i \epsilon$ factors in that term. If we now express $\left\{\left(\beta_{a}-\beta_{b}\right) \ell^{3}+i\left(\epsilon_{1}-\epsilon_{2}\right)\right\}^{-1}$ as a sum of its principal value and a term proportional to $\delta\left(\left(\beta_{a}-\beta_{b}\right) \ell^{3}\right)$, then the contribution to the integral from the principle value term vanishes due to $\ell^{3} \rightarrow-\ell^{3}$ symmetry. The term proportional to $\delta\left(\left(\beta_{a}-\beta_{b}\right) \ell^{3}\right)$ forces $\ell^{3}$ to vanish, in which case the two terms inside the square bracket cancel. Therefore $J_{a b}$ vanishes also in this case.

If $a$ and $b$ both refer to outgoing particles, then the zero of $\ell \cdot p_{a}+i \epsilon$ is in the upper half plane and the zero of $\ell . p_{b}-i \epsilon$ is in the lower half plane. Therefore if we close the contour in the upper half plane so as to avoid contribution from the residues at the poles of $G_{r}(\ell)$, we only pick up the residue at $\ell \cdot p_{a}+i \epsilon=0$. If we choose a frame in which $p_{a}=p_{a}^{0}\left(1,0,0, \beta_{a}\right)$ and $p_{b}=p_{b}^{0}\left(1,0,0, \beta_{b}\right)$, then the pole is at $\ell^{0}=\beta_{a} \ell^{3}+i \epsilon$, and the resulting integrand takes the form

$$
-i \int \frac{d^{3} \ell}{(2 \pi)^{3}} \frac{1}{p_{a}^{0} p_{b}^{0}} \frac{1}{\left(\beta_{a}-\beta_{b}\right) \ell^{3}+i \epsilon} \frac{1}{\left(1-\beta_{a}^{2}\right)\left(\ell^{3}\right)^{2}+\ell_{\perp}^{2}} .
$$


If we express $\left(\left(\beta_{a}-\beta_{b}\right) \ell^{3}+i \epsilon\right)^{-1}$ term as a sum of its principal value and $-i \pi \delta\left(\left(\beta_{a}-\beta_{b}\right) \ell^{3}\right)$, then the contribution from the principal value part vanishes due to $\ell^{3} \rightarrow-\ell^{3}$ symmetry. The term proportional to $\delta\left(\left(\beta_{a}-\beta_{b}\right) \ell^{3}\right)$ forces $\ell^{3}$ to vanish. Integration over $\ell_{\perp} \equiv\left(\ell^{1}, \ell^{2}\right)$ in the range $\omega \ll\left|\ell_{\perp}\right| \ll L^{-1}$ now generates a factor of $2 \pi \ln (\omega L)^{-1}$. This gives

$$
J_{a b}=\frac{1}{4 \pi} \ln (\omega L) \frac{1}{p_{a}^{0} p_{b}^{0}} \frac{1}{\left|\beta_{a}-\beta_{b}\right|}=\frac{1}{4 \pi} \ln (\omega L) \frac{1}{\sqrt{\left(p_{a} \cdot p_{b}\right)^{2}-p_{a}^{2} p_{b}^{2}}},
$$

where in the last step we have reexpressed the result in the covariant form.

If $a$ and $b$ both refer to incoming particles, then the zero of $\ell \cdot p_{a}+i \epsilon$ is in the lower half plane and the zero of $\ell . p_{b}-i \epsilon$ is in the upper half plane. Therefore if we close the contour in the upper half plane, we only pick up the residue at $\ell \cdot p_{b}-i \epsilon=0$. The integral can be evaluated similarly using the same frame as used above and yields the same result (B.4).

We can also determine the $i \epsilon$ prescription for the $\ln \omega$ term by noting that in (B.1), the factor

$$
\left\{(k-\ell) \cdot p_{a}-i \epsilon\right\}^{-1}=\left\{-p_{a}^{0} \omega+\vec{p}_{a} \cdot \vec{k}-\ell \cdot p_{a}-i \epsilon\right\}^{-1}
$$

preserves the $i \epsilon$ prescription under addition of a positive (negative) imaginary part to $\omega$ for positive (negative) $p_{a}^{0}$. Therefore the singularity in the complex $\omega$ plane must be located in the lower (upper) half plane for positive (negative) $p_{a}^{0}$. This shows that $\ln \omega$ in (B.4) stands for $\ln \left(\omega+i \epsilon \eta_{a}\right)$ where $\eta_{a}=1$ for outgoing particles and $\eta_{a}=-1$ for incoming particles. The final result may be written as:

$$
J_{a b}=\frac{1}{4 \pi} \delta_{\eta_{a}, \eta_{b}} \ln \left\{\left(\omega+i \epsilon \eta_{a}\right) L\right\} \frac{1}{\sqrt{\left(p_{a} \cdot p_{b}\right)^{2}-p_{a}^{2} p_{b}^{2}}} .
$$

Next we consider the integral:

$$
K_{b} \equiv 2 \int \frac{d^{4} \ell}{(2 \pi)^{4}} G_{r}(k-\ell) \frac{1}{p_{b} \cdot \ell-i \epsilon} G_{r}(\ell) \simeq \int \frac{d^{4} \ell}{(2 \pi)^{4}} \frac{1}{k \cdot \ell+i \epsilon} \frac{1}{p_{b} \cdot \ell-i \epsilon} \frac{1}{\left(\ell^{0}+i \epsilon\right)^{2}-\vec{\ell}^{2}},
$$

with $\omega$ providing the upper cut-off to the integral and $R^{-1}$ providing the lower cut-off. The last expression is obtained by making the approximation $\left|\ell^{\mu}\right| \ll \omega$ since the logarithmic contribution arises from this region. This integral has the same structure as (B.1) with $p_{a}$ replaced by $k$ and can be evaluated similarly. There are however a few differences:

1. Due to the changes in the cut-off, $\ln (\omega L)$ factor in (B.4) will be replaced by $-\ln (\omega R)$.

2. The $i \epsilon$ prescription for the integral can be determined by noting that in the expression for $G_{r}(k-\ell)=\left\{\left(k^{0}-\ell^{0}+i \epsilon\right)^{2}-(\vec{k}-\vec{\ell})^{2}\right\}^{-1}$ in (B.7), if we add a positive imaginary part to $k^{0}=\omega$ then it does not change the $i \epsilon$ prescription for the poles, but adding a negative imaginary part will change the $i \epsilon$ prescription. Therefore the factors of $\ln \omega$ will correspond to $\ln (\omega+i \epsilon)$.

3. Since $k$ represents an outgoing momentum, it follows from the arguments given below (B.2) that in order for the integral in (B.7) to be non-vanishing, $p_{b}$ must also represent an outgoing momentum. 
4. Since $k^{2}=0$, the denominator factor in (B.6) simplifies to

$$
\sqrt{\left(k \cdot p_{b}\right)^{2}-k^{2} p_{b}^{2}}=-k \cdot p_{b}
$$

with the minus sign arising from the fact that when $k$ and $p_{b}$ both represent outgoing momenta, $k \cdot p_{b}$ is negative.

With these ingredients we can express the final result for $K_{b}$ as:

$$
K_{b}=\frac{1}{4 \pi} \delta_{\eta_{b}, 1} \ln \{(\omega+i \epsilon) R\} \frac{1}{k \cdot p_{b}} .
$$

Finally we shall analyze the integral

$$
K_{b}^{\prime} \equiv 2 \int \frac{d^{4} \ell}{(2 \pi)^{4}} G_{r}(k-\ell) \frac{1}{p_{b} \cdot \ell-i \epsilon} G_{r}(\ell)^{*} \simeq \int \frac{d^{4} \ell}{(2 \pi)^{4}} \frac{1}{k \cdot \ell+i \epsilon} \frac{1}{p_{b} \cdot \ell-i \epsilon} \frac{1}{\left(\ell^{0}-i \epsilon\right)^{2}-\vec{\ell}^{2}}
$$

with $\omega$ providing the upper cut-off to the integral and $R^{-1}$ providing the lower cut-off. To evaluate this integral, note that $\left(K_{b}^{\prime}\right)^{*}$ is formally equal to $K_{b}$ with $\left(k, p_{b}\right)$ replaced by $\left(-k,-p_{b}\right)$. The latter result can be read out for those of $J_{a b}$ with incoming momenta. This gives

$$
K_{b}^{\prime}=\frac{1}{4 \pi} \delta_{\eta_{b}, 1} \ln \{(\omega+i \epsilon) R\} \frac{1}{k \cdot p_{b}} .
$$

\section{Contribution from real gravitons}

In the analysis in section 3.5, we had left out the contribution of the second term of (3.44) in (3.41). This is given by:

$$
\begin{aligned}
\widehat{T}_{\mathrm{extra}}^{\mu \nu}(k)= & 16 i \pi^{2} G \sum_{a, b} \int \frac{d^{4} \ell}{(2 \pi)^{4}} G_{r}(k-\ell) \delta\left(\ell^{2}\right)\left\{H\left(\ell^{0}\right)-H\left(-\ell^{0}\right)\right\} \\
& \times \frac{1}{p_{b} \cdot \ell-i \epsilon} \frac{1}{p_{a} \cdot(k-\ell)-i \epsilon} \mathcal{F}^{\mu \nu, \alpha \beta, \rho \sigma}(k, \ell)\left\{p_{b \alpha} p_{b \beta}-\frac{1}{2} p_{b}^{2} \eta_{\alpha \beta}\right\}\left\{p_{a \rho} p_{a \sigma}-\frac{1}{2} p_{a}^{2} \eta_{\rho \sigma}\right\},
\end{aligned}
$$

where $\mathcal{F}^{\mu \nu, \alpha \beta, \rho \sigma}(k, \ell)$ has been defined in (3.42), and it is understood that the integration over the momenta $\ell^{\mu}$ is restricted to the range much below the cut-off $L^{-1}$, so that we can drop the exponential factors of $e^{-i k \cdot r_{a}}$ and $e^{i \ell \cdot\left(r_{a}-r_{b}\right)}$ that regulate the ultraviolet divergence in (3.41). We shall now analyze possible logarithmic contributions to this term from different regions of integration.

First of all, since each term in $\mathcal{F}^{\mu \nu, \alpha \beta, \rho \sigma}(k, \ell)$ defined in (3.42) has a factor of $(k-\ell)$, a simple power counting shows that there are no logarithmic contributions from the region $\left|k^{\mu}-\ell^{\mu}\right| \ll \omega$. Therefore we need to analyze contributions from the regions $R^{-1} \ll$ $\left|\ell^{\mu}\right| \ll \omega$ and $\omega \ll\left|\ell^{\mu}\right| \ll L^{-1}$. Power counting shows that in order to analyze logarithmic contribution from the region $R^{-1} \ll\left|\ell^{\mu}\right| \ll \omega$, we can replace the numerator by its $\ell \rightarrow 0$ limit. Therefore we need to analyze an integral of the form

$$
\mathcal{E}_{0}=\int \frac{d^{4} \ell}{(2 \pi)^{4}} \frac{1}{p_{b} \cdot \ell-i \epsilon} \frac{1}{p_{a} \cdot(k-\ell)-i \epsilon} G_{r}(k-\ell) \delta\left(\ell^{2}\right)\left\{H\left(\ell^{0}\right)-H\left(-\ell^{0}\right)\right\} .
$$


This can be reexpressed as:

$$
\mathcal{E}_{0}=\frac{1}{2 \pi i} \int \frac{d^{4} \ell}{(2 \pi)^{4}} \frac{1}{p_{b} \cdot \ell-i \epsilon} \frac{1}{p_{a} \cdot(k-\ell)-i \epsilon} G_{r}(k-\ell)\left\{G_{r}(\ell)^{*}-G_{r}(\ell)\right\} .
$$

For $\left|\ell^{\mu}\right| \ll \omega$ the contribution reduces to one of the integrals defined in (B.7) or (B.10) and can be evaluated using (B.9) or (B.11). The result vanishes due to the cancellation between the contributions coming from the $G_{r}(\ell)$ and $G_{r}(\ell)^{*}$ terms. Therefore there is no logarithmic contribution from the $\left|\ell^{\mu}\right| \ll \omega$ region.

We now focus on the region $\left|\ell^{\mu}\right| \gg \omega$. Power counting shows that the integral has linear divergence in this region. So we have to evaluate it carefully by keeping also the subleading terms in this limit. First let us consider the subleading contribution arising from the terms in $\mathcal{F}^{\mu \nu, \alpha \beta, \rho \sigma}(k, \ell)$ that are linear in $\ell$. These involve integrals of the form:

$$
\mathcal{E}_{1}=\int \frac{d^{4} \ell}{(2 \pi)^{4}} \frac{1}{p_{b} \cdot \ell-i \epsilon} \frac{1}{p_{a} \cdot(k-\ell)-i \epsilon} G_{r}(k-\ell) \delta\left(\ell^{2}\right)\left\{H\left(\ell^{0}\right)-H\left(-\ell^{0}\right)\right\} \ell^{\kappa} .
$$

In the region $\left|\ell^{\mu}\right| \gg \omega$, we can approximate the integral as:

$$
\mathcal{E}_{1} \simeq-\int \frac{d^{4} \ell}{(2 \pi)^{4}} \frac{1}{p_{b} \cdot \ell-i \epsilon} \frac{1}{p_{a} \cdot \ell+i \epsilon} \frac{1}{2 k \cdot \ell-i \epsilon \ell^{0}} \delta\left(\ell^{2}\right)\left\{H\left(\ell^{0}\right)-H\left(-\ell^{0}\right)\right\} \ell^{\kappa} .
$$

Now, since $\delta\left(\ell^{2}\right)$ factor puts the momentum $\ell$ on-shell, $p_{a} \cdot \ell$ and $p_{b} . \ell$ never vanish in the integration region of interest and therefore we can drop the $i \epsilon$ factors. ${ }^{10} k$. $\ell$ can vanish only when $\ell$ is parallel to $k$, but by examining the numerator factor (3.42) we find that there are always additional suppression factors in this limit that kill potential singularity at $k . \ell=0$. Therefore the $i \epsilon \ell^{0}$ factor can be dropped from this term as well. For example the presence of a $p_{a} . k$ or $p_{a} \cdot \ell$ factor in the numerator will mean that the ratio $p_{a} \cdot k / p_{a} \cdot \ell$ or $p_{a} \cdot \ell / p_{a} \cdot \ell$ becomes $a$ independent in the limit when $\ell$ is parallel to $k$, and the result then vanishes after summing over $a$ using momentum conservation $\sum_{a} p_{a}=0$. A similar result holds for terms proportional to $p_{b} . k$ or $p_{b} . \ell$. Also, a combination of terms of the form $k^{\mu} \xi^{\nu}+\xi^{\nu} k^{\mu}-k . \xi \eta^{\mu \nu}$ will produce a term in the gravitational wave-form that is pure gauge and therefore can be removed. Therefore we can remove such terms appearing at the level of the integrand itself. Once the $i \epsilon$ factors are removed from all the denominators, the integrand of (C.5) becomes an odd function of $\ell$ and therefore vanishes after integration over $\ell$.

We now turn to the contribution from terms in $\mathcal{F}^{\mu \nu, \alpha \beta, \rho \sigma}(k, \ell)$ that are quadratic in $\ell$. The corresponding integrals take the form:

$$
\mathcal{E}_{2}=\int \frac{d^{4} \ell}{(2 \pi)^{4}} \frac{1}{p_{b} \cdot \ell-i \epsilon} \frac{1}{p_{a} \cdot(k-\ell)-i \epsilon} G_{r}(k-\ell) \delta\left(\ell^{2}\right)\left\{H\left(\ell^{0}\right)-H\left(-\ell^{0}\right)\right\} \ell^{\kappa} \ell^{\tau} .
$$

This has potential linear divergence from the region $\left|\ell^{\mu}\right| \gg \omega$. Therefore we need to expand the $\left(p_{a} .(k-\ell)-i \epsilon\right)^{-1}$ factor in powers of $p_{a} . k$ to the first subleading order:

$$
\frac{1}{p_{a} \cdot(k-\ell)-i \epsilon}=-\frac{1}{p_{a} \cdot \ell+i \epsilon}-\frac{p_{a} \cdot k}{\left(p_{a} \cdot \ell+i \epsilon\right)^{2}} .
$$

\footnotetext{
${ }^{10}$ The only exception is when $p_{a}$ and / or $p_{b}$ represents a massless particle and $\ell$ becomes parallel to $p_{a}$ and / or $p_{b}$ producing a collinear divergence; but such divergences are known to cancel in gravitational theories [66].
} 
We can argue as before that due to the presence of the $\delta\left(\ell^{2}\right)$ factor we can drop all the $i \epsilon$ factors in the denominator. In this case the contribution from the last term in (C.7) to the integral (C.6) vanishes by $\ell \rightarrow-\ell$ symmetry. On the other hand, when we substitute the first term on the right hand side of (C.7) into (C.6), the integrand is an even function of $\ell$. In this case terms proportional to $H\left(\ell^{0}\right)$ and $-H\left(-\ell^{0}\right)$ give identical contributions, and we get:

$$
\mathcal{E}_{2} \simeq-\int \frac{d^{4} \ell}{(2 \pi)^{4}} \frac{1}{p_{b} \cdot \ell-i \epsilon} \frac{1}{p_{a} \cdot \ell+i \epsilon} \frac{1}{k \cdot \ell-i \epsilon} \delta\left(\ell^{2}\right) H\left(\ell^{0}\right) \ell^{\kappa} \ell^{\tau}
$$

Note that we have kept the $i \epsilon$ factors even though the presence of $\delta\left(\ell^{2}\right)$ makes them irrelevant.

Now from (C.1) and (3.42) we see that the indices $\kappa$ and $\tau$ must either be free indices $\mu, \nu$, or be contracted with the index of $p_{a}$ or $p_{b}$, or be contracted with each other. If they are contracted with each other then we have a factor of $\ell^{2}$ and the contribution vanishes due to the $\delta\left(\ell^{2}\right)$ factor. If any one of them is contracted with $p_{b}$, then we have a factor of $p_{b} . \ell$ in the numerator that kills the denominator factor of $p_{b} . \ell-i \epsilon$. After summing over $b$ and using momentum conservation law $\sum_{b} p_{b}=0$, this contribution also vanishes. A similar argument can be given for terms where either $\ell^{\kappa}$ or $\ell^{\tau}$ is contracted with $p_{a}$. The only term that survives is where $(\kappa, \tau)$ take values $(\mu, \nu)$. Using this we can bring the contribution to (C.1) to the form

$$
\widehat{T}_{\text {extra }}^{\mu \nu}=\frac{G}{\pi^{2}} \int\left\{d^{4} \ell \delta\left(\ell^{2}\right) H\left(\ell^{0}\right)\right\}\left\{\sum_{a, b=1}^{m+n} \frac{1}{\left(p_{a} \cdot \ell-i \epsilon\right)\left(p_{b} \cdot \ell+i \epsilon\right)}\right\}\left\{\left(p_{a} \cdot p_{b}\right)^{2}-\frac{1}{2} p_{a}^{2} p_{b}^{2}\right\} \frac{\ell^{\mu} \ell^{\nu}}{i(k \cdot \ell-i \epsilon)}
$$

We shall now show that this contribution can be interpreted as the effect of soft emission from the gravitational radiation produced during the scattering, and is therefore already accounted for when we include in the sum over $a$ in the soft factor the contribution from the gravitational radiation produced during the scattering. For this we note that the flux of radiation in a phase space volume $\delta\left(\ell^{2}\right) H\left(\ell^{0}\right) d^{4} \ell$ carrying polarization $\varepsilon_{\mu \nu}$ is given by

$$
\frac{G}{\pi^{2}}\left\{d^{4} \ell \delta\left(\ell^{2}\right) H\left(\ell^{0}\right)\right\}\left\{\sum_{a=1}^{m+n} \frac{p_{a}^{\rho} p_{a}^{\sigma}}{p_{a} \cdot \ell-i \epsilon}\right\}\left\{\sum_{b=1}^{m+n} \frac{p_{b}^{\kappa} p_{b}^{\tau}}{p_{b} \cdot \ell+i \epsilon}\right\}\left(\varepsilon_{\kappa \tau}\right)^{*} \varepsilon_{\rho \sigma} .
$$

This equation can be derived by using the relation between the leading soft factor (3.17) and the flux of radiation [15]. In this case the first factor inside the curly bracket gives the phase space volume, and the rest of the factors gives the flux of radiation produced in the scattering. Since we shall be interested in only the total flux, we can sum over polarizations using the formula

$$
\sum_{\varepsilon}\left(\varepsilon_{\kappa \tau}\right)^{*} \varepsilon_{\rho \sigma}=\frac{1}{2}\left(\eta_{\kappa \rho} \eta_{\tau \sigma}+\eta_{\kappa \sigma} \eta_{\tau \rho}-\eta_{\kappa \tau} \eta_{\rho \sigma}\right)
$$


yielding the standard result for the total flux of gravitational radiation given e.g. in eq. (10.4.22) of [55]:

$$
\frac{G}{\pi^{2}}\left\{d^{4} \ell \delta\left(\ell^{2}\right) H\left(\ell^{0}\right)\right\}\left\{\sum_{a=1}^{m+n} \frac{p_{a}^{\rho} p_{a}^{\sigma}}{p_{a} \cdot \ell-i \epsilon}\right\}\left\{\sum_{b=1}^{m+n} \frac{p_{b}^{\kappa} p_{b}^{\tau}}{p_{b} \cdot \ell+i \epsilon}\right\} \frac{1}{2}\left(\eta_{\kappa \rho} \eta_{\tau \sigma}+\eta_{\kappa \sigma} \eta_{\tau \rho}-\eta_{\kappa \tau} \eta_{\rho \sigma}\right) .
$$

The leading soft theorem (3.17), applied to this radiation flux, now shows that the contribution to the $\widehat{T}^{\mu \nu}$ due to the radiation is obtained by multiplying (C.12) by $-i /(\ell . k-i \epsilon)$ and integrating over $\ell$. This gives the net leading contribution to the soft factor due to radiation to be

$$
\widehat{T}^{R \mu \nu}=\frac{G}{\pi^{2}} \int\left\{d^{4} \ell \delta\left(\ell^{2}\right) H\left(\ell^{0}\right)\right\}\left\{\sum_{a, b=1}^{m+n} \frac{1}{\left(p_{a} \cdot \ell-i \epsilon\right)\left(p_{b} \cdot \ell+i \epsilon\right)}\right\}\left\{\left(p_{a} \cdot p_{b}\right)^{2}-\frac{1}{2} p_{a}^{2} p_{b}^{2}\right\} \frac{\ell^{\mu} \ell^{\nu}}{i(k \cdot \ell-i \epsilon)} .
$$

This agrees with (C.9), showing that the extra contribution (C.1) is already accounted for by including in the sum over $a$ in the soft factor the contribution due to radiation. ${ }^{11}$

\section{Position space analysis of $\widehat{T}^{X \mu \nu}$}

In section 3, section 4 we have carried out our analysis in momentum space. This has the advantage that the expressions we obtain are similar to the ones that appear in the evaluation of Feynman diagrams, and various general techniques developed for computing amplitudes in quantum field theory may find applications here. Nevertheless it is instructive to see how some of these computations can also be performed directly in position space. In this appendix we shall show how to carry out the analysis of sections section 3.3 and section 3.4 directly in position space.

Our first task will be to compute the gravitational fields produced by the incoming and outgoing particles during a scattering, and study their effect on the motion of the other particles. At the leading order, the incoming and outgoing particle trajectories are given by (3.11), or equivalently (3.15). Using retarded Green's function in flat space-time, we get the following expression for the gravitational field produced by the $b$-th particle on the forward light-cone of the trajectory of the particle [18]:

$$
e_{\mu \nu}^{(b)}(x)=2 G m_{b} \frac{v_{b \mu} v_{b \nu}}{\sqrt{\left(v_{b} \cdot x\right)^{2}+x^{2}}}, \quad h_{\mu \nu}^{(b)}=e_{\mu \nu}^{(b)}-\frac{1}{2} \eta_{\mu \nu} e_{\rho}^{(b) \rho} .
$$

The associated Christoffel symbol is given by, in the weak field approximation,

$$
\begin{aligned}
\Gamma_{\rho \tau}^{(b) \alpha}(x)= & -2 G m_{b} \frac{1}{\left\{\left(v_{b} \cdot x\right)^{2}+x^{2}\right\}^{3 / 2}} \eta^{\alpha \mu}\left[\left\{v_{b \mu} v_{b \tau}+\frac{1}{2} \eta_{\mu \tau}\right\}\left\{x_{\rho}+v_{b} \cdot x v_{b \rho}\right\}\right. \\
& \left.+\left\{v_{b \mu} v_{b \rho}+\frac{1}{2} \eta_{\mu \rho}\right\}\left\{x_{\tau}+v_{b} . x v_{b \tau}\right\}-\left\{v_{b \rho} v_{b \tau}+\frac{1}{2} \eta_{\rho \tau}\right\}\left\{x_{\mu}+v_{b} . x v_{b \mu}\right\}\right] .
\end{aligned}
$$

\footnotetext{
${ }^{11}$ As in [15], this can also be expressed as angular integrals over appropriate functions of the radiative gravitational field and its derivatives, but we shall not describe this here.
} 
Since the field has support on the forward light-cone of the trajectory, it follows that in sufficiently far future and far past of the scattering event, the outgoing particles are affected by the gravitational field of the outgoing particles and the incoming particles are affected by the gravitational field of the incoming particles.

Let $Y_{a}^{\mu}$ denote the correction to the particle trajectory (3.15) due to the gravitational field produced by the other particles:

$$
X_{a}^{\mu}(\sigma)=v_{a}^{\mu} \sigma+r_{a}^{\mu}+Y_{a}^{\mu}(\sigma) .
$$

We shall use the compact notation described in (3.12), and define $\eta_{a}$ to be a number that takes value 1 for outgoing particles $(1 \leq a \leq n)$ and -1 for incoming particles $(n+1 \leq a \leq m+n)$. Then $Y_{a}^{\mu}$ satisfies the differential equation and boundary conditions:

$$
\frac{d^{2} Y_{a}^{\mu}}{d \sigma^{2}}=-\Gamma_{\nu \rho}^{\mu}\left(v_{a} \sigma+r_{a}\right) v_{a}^{\mu} v_{a}^{\nu}, \quad Y_{a}^{\mu} \rightarrow 0 \text { as } \sigma \rightarrow 0, \quad \frac{d Y_{a}^{\mu}}{d \sigma} \rightarrow 0 \text { as } \sigma \rightarrow \infty,
$$

where

$$
\Gamma_{\nu \rho}^{\mu}=\sum_{\substack{b=1 \\ b \neq a, \eta_{a} \eta_{b}=1}}^{m+n} \Gamma_{\nu \rho}^{(b) \mu} .
$$

The constraint $\eta_{a} \eta_{b}=1$ reflects that the outgoing particles are affected by the gravitational field of the outgoing particles and the incoming particles are affected by the gravitational field of the incoming particles. Using (D.2), (D.4) and (D.5) we get, for $\sigma \gg\left|r_{a}\right| \sim L$ :

$$
\frac{d^{2} Y_{a}^{\alpha}(\sigma)}{d \sigma^{2}} \simeq \frac{2 G}{\sigma^{2}} \sum_{\substack{b=1 \\ b \neq a, \eta_{a} \eta_{b}=1}}^{m+n} m_{b} \frac{1}{\left\{\left(v_{b} \cdot v_{a}\right)^{2}-1\right\}^{3 / 2}}\left[-\frac{1}{2} v_{a}^{\alpha}+\frac{1}{2} v_{b}^{\alpha}\left\{2\left(v_{b} \cdot v_{a}\right)^{3}-3 v_{b} \cdot v_{a}\right\}\right]
$$

This gives

$$
\frac{d Y_{a}^{\alpha}(\sigma)}{d \sigma} \simeq-\frac{2 G}{\sigma} \sum_{\substack{b=1 \\ b \neq a, \eta_{a} \eta_{b}=1}}^{m+n} m_{b} \frac{1}{\left\{\left(v_{b} \cdot v_{a}\right)^{2}-1\right\}^{3 / 2}}\left[-\frac{1}{2} v_{a}^{\alpha}+\frac{1}{2} v_{b}^{\alpha}\left\{2\left(v_{b} \cdot v_{a}\right)^{3}-3 v_{b} \cdot v_{a}\right\}\right] .
$$

Now in (3.28) we have the expression for $\widehat{T}_{\mu \nu}^{X}$ to subleading order:

$$
\widehat{T}^{X \mu \nu}(k)=\sum_{a=1}^{m+n} m_{a} \int_{0}^{\infty} d \sigma e^{-i k \cdot\left(v_{a} \sigma+r_{a}\right)}\left[v_{a}^{\mu} v_{a}^{\nu}-i k \cdot Y_{a}(\sigma) v_{a}^{\mu} v_{a}^{\nu}+\frac{d Y_{a}^{\mu}}{d \sigma} v_{a}^{\nu}+v_{a}^{\mu} \frac{d Y_{a}^{\nu}}{d \sigma}\right] .
$$

As discussed below (3.17), the integration over $\sigma$ is made well defined by replacing $\omega$ by $\omega+i \epsilon$ for outgoing particles and by $\omega-i \epsilon$ for incoming particles. We now manipulate the second term by writing

$$
e^{-i k \cdot\left(v_{a} \sigma+r_{a}\right)}=\frac{i}{k \cdot v_{a}} \frac{d}{d \sigma} e^{-i k \cdot\left(v_{a} \sigma+r_{a}\right)}
$$

and integrating over $\sigma$ by parts. The boundary term at infinity vanishes due to the replacement of $\omega$ by $\omega+i \epsilon \eta_{a}$, while the boundary term at $\sigma=0$ gives a finite contribution 
in the $\omega \rightarrow 0$ limit and is not of interest to us. With this (D.8) can be expressed as

$$
\widehat{T}^{X \mu \nu}(k)=\sum_{a=1}^{m+n} m_{a} \int_{0}^{\infty} d \sigma e^{-i k \cdot\left(v_{a} \sigma+r_{a}\right)}\left[v_{a}^{\mu} v_{a}^{\nu}-\frac{1}{k \cdot v_{a}} k \cdot \frac{d Y_{a}}{d \sigma} v_{a}^{\mu} v_{a}^{\nu}+\frac{d Y_{a}^{\mu}}{d \sigma} v_{a}^{\nu}+v_{a}^{\mu} \frac{d Y_{a}^{\nu}}{d \sigma}\right] .
$$

After integration over $\sigma$ the first term gives the leading term. In the other terms we can substitute the expression (D.7) for $d Y_{a} / d \sigma$. Since the integrand is proportional to $1 / \sigma$ in the range $L \ll \sigma \ll \omega^{-1}$, we get contribution proportional to $\ln \left(\left(\omega+i \epsilon \eta_{a}\right)^{-1} / L\right)$. Therefore, with the help of (D.7), the logarithmic correction to $\widehat{T}^{X}$, given by the last three terms in (D.10), takes the form:

$$
\begin{aligned}
\Delta \widehat{T}^{X \mu \nu}(k)=2 G & \sum_{a=1}^{m+n} m_{a} \ln \left\{L\left(\omega+i \epsilon \eta_{a}\right)\right\} \sum_{\substack{b=1 \\
b \neq a, \eta_{a} \eta_{b}=1}}^{m+n} m_{b} \frac{1}{\left\{\left(v_{b} \cdot v_{a}\right)^{2}-1\right\}^{3 / 2}} \\
\times[ & -\frac{v_{a}^{\mu} v_{a}^{\nu}}{k \cdot v_{a}} k_{\alpha}\left\{-\frac{1}{2} v_{a}^{\alpha}+\frac{1}{2} v_{b}^{\alpha}\left\{2\left(v_{b} \cdot v_{a}\right)^{3}-3 v_{b} \cdot v_{a}\right\}\right\} \\
& +\left\{-\frac{1}{2} v_{a}^{\mu}+\frac{1}{2} v_{b}^{\mu}\left\{2\left(v_{b} \cdot v_{a}\right)^{3}-3 v_{b} \cdot v_{a}\right\}\right\} v_{a}^{\nu} \\
& \left.+\left\{-\frac{1}{2} v_{a}^{\nu}+\frac{1}{2} v_{b}^{\nu}\left\{2\left(v_{b} \cdot v_{a}\right)^{3}-3 v_{b} \cdot v_{a}\right\}\right\} v_{a}^{\mu}\right] .
\end{aligned}
$$

After using the relations $p_{a}=m_{a} v_{a}$ and some simplification we get:

$$
\begin{aligned}
\Delta \widehat{T}^{X \mu \nu}(k)=2 G & \sum_{a=1}^{m+n} \ln \left\{L\left(\omega+i \epsilon \eta_{a}\right)\right\} \sum_{\substack{b=1 \\
b \neq a, \eta_{a} \eta_{b}=1}}^{m+n} \frac{1}{\left\{\left(p_{a} \cdot p_{b}\right)^{2}-p_{a}^{2} p_{b}^{2}\right\}^{3 / 2}} \\
\times & {\left[\frac{k \cdot p_{b}}{k \cdot p_{a}} p_{a}^{\mu} p_{a}^{\nu} p_{a} \cdot p_{b}\left\{\frac{3}{2} p_{a}^{2} p_{b}^{2}-\left(p_{a} \cdot p_{b}\right)^{2}\right\}\right.} \\
& \left.+\frac{1}{2} p_{a}^{\mu} p_{a}^{\nu} p_{a}^{2}\left(p_{b}^{2}\right)^{2}-\left\{p_{a}^{\mu} p_{b}^{\nu}+p_{a}^{\nu} p_{b}^{\mu}\right\} p_{a} \cdot p_{b}\left\{\frac{3}{2} p_{a}^{2} p_{b}^{2}-\left(p_{a} \cdot p_{b}\right)^{2}\right\}\right] .
\end{aligned}
$$

This is in perfect agreement with (3.39).

Open Access. This article is distributed under the terms of the Creative Commons Attribution License (CC-BY 4.0), which permits any use, distribution and reproduction in any medium, provided the original author(s) and source are credited.

\section{References}

[1] S. Weinberg, Infrared photons and gravitons, Phys. Rev. 140 (1965) B516 [InSPIRE].

[2] C.D. White, Factorization Properties of Soft Graviton Amplitudes, JHEP 05 (2011) 060 [arXiv: 1103.2981] [INSPIRE].

[3] T. He, V. Lysov, P. Mitra and A. Strominger, BMS supertranslations and Weinberg's soft graviton theorem, JHEP 05 (2015) 151 [arXiv:1401.7026] [INSPIRE]. 
[4] F. Cachazo and A. Strominger, Evidence for a New Soft Graviton Theorem, arXiv: 1404.4091 [INSPIRE].

[5] N. Afkhami-Jeddi, Soft Graviton Theorem in Arbitrary Dimensions, arXiv:1405.3533 [INSPIRE].

[6] Z. Bern, S. Davies, P. Di Vecchia and J. Nohle, Low-Energy Behavior of Gluons and Gravitons from Gauge Invariance, Phys. Rev. D 90 (2014) 084035 [arXiv:1406.6987] [INSPIRE].

[7] M. Campiglia and A. Laddha, Asymptotic symmetries and subleading soft graviton theorem, Phys. Rev. D 90 (2014) 124028 [arXiv: 1408.2228] [INSPIRE].

[8] A. Laddha and A. Sen, Sub-subleading Soft Graviton Theorem in Generic Theories of Quantum Gravity, JHEP 10 (2017) 065 [arXiv:1706.00759] [INSPIRE].

[9] F. Cachazo, S. He and E.Y. Yuan, New Double Soft Emission Theorems, Phys. Rev. D 92 (2015) 065030 [arXiv:1503.04816] [INSPIRE].

[10] T. Klose, T. McLoughlin, D. Nandan, J. Plefka and G. Travaglini, Double-Soft Limits of Gluons and Gravitons, arXiv:1504.05558 [INSPIRE].

[11] A.P. Saha, Double Soft Theorem for Perturbative Gravity, JHEP 09 (2016) 165 [arXiv: 1607.02700] [INSPIRE].

[12] S. Chakrabarti, S.P. Kashyap, B. Sahoo, A. Sen and M. Verma, Subleading Soft Theorem for Multiple Soft Gravitons, JHEP 12 (2017) 150 [arXiv:1707.06803] [InSPIRE].

[13] S. Atul Bhatkar and B. Sahoo, Subleading Soft Theorem for arbitrary number of external soft photons and gravitons, JHEP 01 (2019) 153 [arXiv:1809.01675] [INSPIRE].

[14] D. Jain and A. Rudra, Leading soft theorem for multiple gravitini, JHEP 06 (2019) 004 [arXiv: 1811.01804] [INSPIRE].

[15] A. Laddha and A. Sen, Gravity Waves from Soft Theorem in General Dimensions, JHEP 09 (2018) 105 [arXiv: 1801.07719] [InSPIRE].

[16] A. Laddha and A. Sen, Classical proof of the classical soft graviton theorem in Dí4, Phys. Rev. D 101 (2020) 084011 [arXiv: 1906.08288] [INSPIRE].

[17] A. Laddha and A. Sen, Logarithmic Terms in the Soft Expansion in Four Dimensions, JHEP 10 (2018) 056 [arXiv: 1804. 09193] [inSPIRE].

[18] B. Sahoo and A. Sen, Classical and Quantum Results on Logarithmic Terms in the Soft Theorem in Four Dimensions, JHEP 02 (2019) 086 [arXiv: 1808. 03288] [INSPIRE].

[19] A. Laddha and A. Sen, Observational Signature of the Logarithmic Terms in the Soft Graviton Theorem, Phys. Rev. D 100 (2019) 024009 [arXiv: 1806.01872] [InSPIRE].

[20] M. Campiglia and A. Laddha, Loop Corrected Soft Photon Theorem as a Ward Identity, JHEP 10 (2019) 287 [arXiv: 1903.09133] [INSPIRE].

[21] S. Bhatkar, A.P. Saha and B. Sahoo, to appear.

[22] Y.B. Zel'dovich and A.G. Polnarev, Radiation of gravitational waves by a cluster of superdense stars, Sov. Astron. 18 (1974) 17 [INSPIRE].

[23] V.B. Braginsky and L.P. Grishchuk, Kinematic Resonance and Memory Effect in Free Mass Gravitational Antennas, Sov. Phys. JETP 62 (1985) 427 [Zh. Eksp. Teor. Fiz. 89 (1985) 744] [INSPIRE]. 
[24] V.B. Braginsky and K.S. Thorne, Gravitational-wave bursts with memory and experimental prospects, Nature 327 (1987) 123.

[25] M. Ludvigsen, Geodesic Deviation At Null Infinity And The Physical Effects Of Very Long Wave Gravitational Radiation, Gen. Rel. Grav. 21 (1989) 1205 [INSPIRE].

[26] D. Christodoulou, Nonlinear nature of gravitation and gravitational wave experiments, Phys. Rev. Lett. 67 (1991) 1486 [INSPIRE].

[27] K.S. Thorne, Gravitational-wave bursts with memory: The Christodoulou effect, Phys. Rev. D 45 (1992) 520 [INSPIRE].

[28] L. Blanchet and T. Damour, Hereditary effects in gravitational radiation, Phys. Rev. D 46 (1992) 4304 [INSPIRE].

[29] M. Favata, The gravitational-wave memory effect, Class. Quant. Grav. 27 (2010) 084036 [arXiv: 1003.3486] [INSPIRE].

[30] A. Tolish and R.M. Wald, Retarded Fields of Null Particles and the Memory Effect, Phys. Rev. D 89 (2014) 064008 [arXiv: 1401.5831] [InSPIRE].

[31] L. Bieri and D. Garfinkle, Perturbative and gauge invariant treatment of gravitational wave memory, Phys. Rev. D 89 (2014) 084039 [arXiv:1312.6871] [InSPIRE].

[32] A. Strominger and A. Zhiboedov, Gravitational Memory, BMS Supertranslations and Soft Theorems, JHEP 01 (2016) 086 [arXiv:1411.5745] [INSPIRE].

[33] P.C. Peters, Relativistic gravitational bremsstrahlung, Phys. Rev. D 1 (1970) 1559 [INSPIRE].

[34] M. Ciafaloni, D. Colferai and G. Veneziano, Infrared features of gravitational scattering and radiation in the eikonal approach, Phys. Rev. D 99 (2019) 066008 [arXiv:1812.08137] [INSPIRE].

[35] A. Addazi, M. Bianchi and G. Veneziano, Soft gravitational radiation from ultra-relativistic collisions at sub- and sub-sub-leading order, JHEP 05 (2019) 050 [arXiv:1901.10986] [INSPIRE].

[36] W.D. Goldberger and A.K. Ridgway, Radiation and the classical double copy for color charges, Phys. Rev. D 95 (2017) 125010 [arXiv:1611.03493] [INSPIRE].

[37] W.M. Tulczyjew, Motion of multipole particles in general relativity theory binaries, Acta Phys. Polon. 18 (1959) 393.

[38] W.G. Dixon, Extended bodies in general relativity: Their description and motion, in Proceedings of the International School of Physics Enrico Fermi LXVII, edited by J. Ehlers, North Holland, Amsterdam, (1979), pp. 156-219.

[39] R.A. Porto, Post-Newtonian corrections to the motion of spinning bodies in NRGR, Phys. Rev. D 73 (2006) 104031 [gr-qc/0511061] [INSPIRE].

[40] W.D. Goldberger and I.Z. Rothstein, Dissipative effects in the worldline approach to black hole dynamics, Phys. Rev. D $\mathbf{7 3}$ (2006) 104030 [hep-th/0511133] [INSPIRE].

[41] R.A. Porto and I.Z. Rothstein, The Hyperfine Einstein-Infeld-Hoffmann potential, Phys. Rev. Lett. 97 (2006) 021101 [gr-qc/0604099] [INSPIRE].

[42] R.A. Porto and I.Z. Rothstein, Next to Leading Order Spin(1)Spin(1) Effects in the Motion of Inspiralling Compact Binaries, Phys. Rev. D 78 (2008) 044013 [Erratum ibid. 81 (2010) 029905] [arXiv:0804.0260] [INSPIRE]. 
[43] J. Vines, Scattering of two spinning black holes in post-Minkowskian gravity, to all orders in spin and effective-one-body mappings, Class. Quant. Grav. 35 (2018) 084002 [arXiv: 1709.06016] [INSPIRE].

[44] W.D. Goldberger, J. Li and S.G. Prabhu, Spinning particles, axion radiation and the classical double copy, Phys. Rev. D 97 (2018) 105018 [arXiv:1712.09250] [INSPIRE].

[45] A. Guevara, A. Ochirov and J. Vines, Scattering of Spinning Black Holes from Exponentiated Soft Factors, JHEP 09 (2019) 056 [arXiv: 1812.06895] [INSPIRE].

[46] W.D. Goldberger and I.Z. Rothstein, An effective field theory of gravity for extended objects, Phys. Rev. D 73 (2006) 104029 [hep-th/0409156] [inSPIRE].

[47] W.D. Goldberger and A. Ross, Gravitational radiative corrections from effective field theory, Phys. Rev. D 81 (2010) 124015 [arXiv:0912.4254] [InSPIRE].

[48] R.A. Porto, A. Ross and I.Z. Rothstein, Spin induced multipole moments for the gravitational wave amplitude from binary inspirals to 2.5 Post-Newtonian order, JCAP 09 (2012) 028 [arXiv: 1203.2962] [INSPIRE].

[49] W.D. Goldberger, A. Ross and I.Z. Rothstein, Black hole mass dynamics and renormalization group evolution, Phys. Rev. D 89 (2014) 124033 [arXiv:1211.6095] [INSPIRE].

[50] S. Foffa and R. Sturani, Hereditary terms at next-to-leading order in two-body gravitational dynamics, Phys. Rev. D 101 (2020) 064033 [arXiv: 1907.02869] [INSPIRE].

[51] W.D. Goldberger and A.K. Ridgway, Bound states and the classical double copy, Phys. Rev. D 97 (2018) 085019 [arXiv:1711.09493] [INSPIRE].

[52] W.D. Goldberger, S.G. Prabhu and J.O. Thompson, Classical gluon and graviton radiation from the bi-adjoint scalar double copy, Phys. Rev. D 96 (2017) 065009 [arXiv:1705.09263] [INSPIRE].

[53] C.-H. Shen, Gravitational Radiation from Color-Kinematics Duality, JHEP 11 (2018) 162 [arXiv: 1806. 07388] [INSPIRE].

[54] E. Poisson, The motion of point particles in curved space-time, Living Rev. Rel. 7 (2004) 6 [gr-qc/0306052] [INSPIRE].

[55] S. Weinberg, Gravitation and Cosmology: Principles and Applications of the General Theory of Relativity, John Wiley and Sons, New York (1972).

[56] L. Bieri and D. Garfinkle, An electromagnetic analogue of gravitational wave memory, Class. Quant. Grav. 30 (2013) 195009 [arXiv:1307.5098] [INSPIRE].

[57] S. Pasterski, Asymptotic Symmetries and Electromagnetic Memory, JHEP 09 (2017) 154 [arXiv: 1505.00716] [INSPIRE].

[58] L. Susskind, Electromagnetic Memory, arXiv:1507.02584 [INSPIRE].

[59] L. Blanchet and G. Schaefer, Gravitational wave tails and binary star systems, Class. Quant. Grav. 10 (1993) 2699 [INSPIRE].

[60] S. Pasterski, A. Strominger and A. Zhiboedov, New Gravitational Memories, JHEP 12 (2016) 053 [arXiv: 1502.06120] [INSPIRE].

[61] Y.F. Bautista and A. Guevara, From Scattering Amplitudes to Classical Physics: Universality, Double Copy and Soft Theorems, arXiv:1903.12419 [INSPIRE]. 
[62] J.G. Hills, Hyper-velocity and tidal stars from binaries disrupted by a massive Galactic black hole, Nature 331 (1988) 687.

[63] H.-T. Janka, Explosion Mechanisms of Core-Collapse Supernovae, Ann. Rev. Nucl. Part. Sci. 62 (2012) 407 [arXiv: 1206.2503] [inSPIRE].

[64] https://chandra.harvard.edu/photo/2002/0066/.

[65] D. Paraficz et al., The bullet cluster at its best: weighing stars, gas and dark matter, Astron. Astrophys. 594 (2016) A121 [arXiv:1209.0384] [INSPIRE].

[66] R. Akhoury, R. Saotome and G. Sterman, Collinear and Soft Divergences in Perturbative Quantum Gravity, Phys. Rev. D 84 (2011) 104040 [arXiv:1109.0270] [InSPIRE]. 